\title{
Selective Synthesis of Spiro[4,5]trienyl Acetates via An Intramolecular Electrophilic ipso- lodocyclization Process
}

Bo-Xiao Tang, ${ }^{\dagger}$ Dong-Jun Tang, ${ }^{\dagger}$ Shi Tang, ${ }^{\dagger}$ Quan-Fu Yu, ${ }^{\dagger}$ Yue-Hua Zhang, ${ }^{\dagger}$ Yun Liang, ${ }^{\dagger}$ Ping Zhong," Jin-Heng Li*

Key Laboratory of Chemical Biology \& Traditional Chinese Medicine Research, Hunan Normal University, Changsha 410081, China, Technical Institute of Physics and Chemistry, Chinese Academy of Scicence, Beijing, 100080, China and Graduate School of Chinese Acedemy of Scineces, Beijing, China, and College of Chemistry and Materials Science, Wenzhou University, Wenzhou 325035, China

jhli@hunnu.edu.cn

\section{Supporting Information \\ List of Contents}

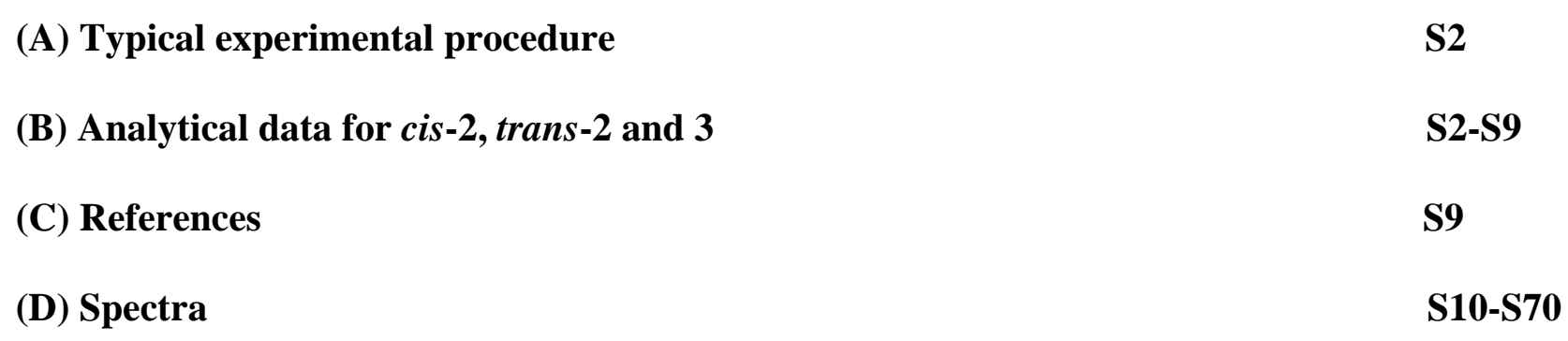




\section{(A) Typical experimental procedure}

\section{(a) Materials:}

Substrates 1a-v were prepared according to the known procedures. ${ }^{1}$

(b) Typical experimental procedure for the intramolecular ipso-iodocyclizations of 4-(punsubstitutedaryl)-1-alkynes (1):

A mixture of amide 1 (0.4 mmol) and NIS (1.5 equiv) in HOAc (1 mL) was stirred at room temperature for $3 \mathrm{~h}$ until complete consumption of starting material as monitored by TLC and GC-MS analysis. After the reaction was finished, the mixture was poured into ethyl acetate, which was washed with $20 \%$ aqueous $\mathrm{NaOH}(2 \times 5 \mathrm{~mL})$, and then with saturated $\mathrm{NaHCO}_{3}(2 \times 5 \mathrm{~mL})$. The aqueous layer was extracted with ethyl acetate and the combined organic layers was dried over anhydrous $\mathrm{Na}_{2} \mathrm{SO}_{4}$ and evaporated under vacuum. The residue was purified by flash column chromatography (hexane/ethyl acetate) to afford the desired product 2 or 3.

\section{(B) Analytical data for cis-2, trans-2 and 3}

cis-N-Methyl-3-iodo-4-phenyl-1-azaspiro[4,5]-deca-3,6,9-trienyl 8-acetate (2a): White solid, mp

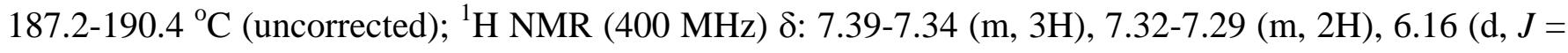
$10.0 \mathrm{~Hz}, 2 \mathrm{H}), 5.59$ (d, $J=10.0 \mathrm{~Hz}, 2 \mathrm{H}), 5.28$ (s, 1H), 2.93 (s, 3H), 2.08 (s, 3H); ${ }^{13} \mathrm{C}$ NMR (100 MHz) $\delta:$ 170.3, 162.1, 146.3, 133.1, 130.4, 129.5, 128.4, 127.9, 96.7, 69.1, 63.3, 26.5, 21.0; IR (KBr, $\left.\mathrm{cm}^{-1}\right)$ : 1744, 1695, 1220; LRMS (EI, $70 \mathrm{eV)} \mathrm{m/z} \mathrm{( \% ):} 421$ (M+1 3), 379 (4), 294 (42), 129 (100); HRMS (EI) for $\mathrm{C}_{18} \mathrm{H}_{16} \mathrm{INO}_{3}\left(\mathrm{M}^{+}\right)$: calcd. 421.0175, found 421.0175.

trans-N-Methyl-3-iodo-4-phenyl-1-azaspiro[4,5]-deca-3,6,9-trienyl 8-acetate (2a): White solid, mp

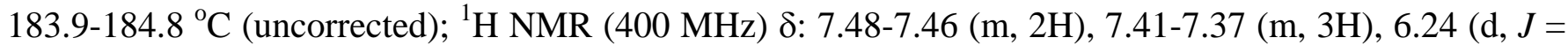
$11.2 \mathrm{~Hz}, 2 \mathrm{H}), 5.64$ (d, $J=9.0 \mathrm{~Hz}, 3 \mathrm{H}), 2.84$ (s, 3H), 1.98 (s, 3H); ${ }^{13} \mathrm{C}$ NMR (100 MHz) $\delta: ~ 170.4, ~ 167.2$, 161.1, 132.8, 130.3, 129.6, 129.2, 128.3, 128.2, 96.4, 68.8, 62.9, 29.7, 26.2, 20.9; IR (KBr, $\left.\mathrm{cm}^{-1}\right): 1727$, 1669, 1235; LRMS (EI, $70 \mathrm{eV)} \mathrm{m/z} \mathrm{( \% ):} 421$ (M+19), 379 (10), 294 (100), 129 (98); HRMS (EI) for $\mathrm{C}_{18} \mathrm{H}_{16} \mathrm{INO}_{3}\left(\mathrm{M}^{+}\right)$: calcd. 421.0175, found 421.0175. 
cis-N-Methyl-3-iodo-4-phenyl-6-methyl-1-azaspiro[4,5]-deca-3,6,9-trienyl 8-acetate (2e): White

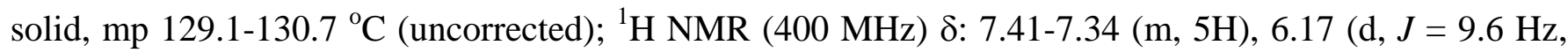
1H), 5.91 (s, 1H), 5.55 (d, $J=9.6 \mathrm{~Hz}, 1 \mathrm{H}), 5.37$ (s, 1H), 2.87 (s, 3H), 2.08 (s, 3H), 1.57 (s, 3H); ${ }^{13} \mathrm{C}$ NMR

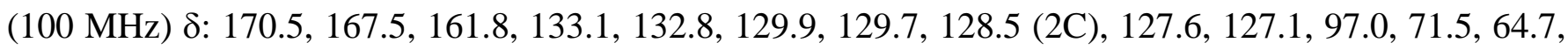
26.2, 21.1, 17.0; IR (KBr, $\left.\mathrm{cm}^{-1}\right)$ : 1731, 1694, 1232; LRMS (EI, $\left.70 \mathrm{eV}\right) \mathrm{m} / \mathrm{z}(\%): 435\left(\mathrm{M}^{+}, 8\right), 393$ (35), 308 (91), 129 (100); HRMS (EI) for $\mathrm{C}_{19} \mathrm{H}_{18} \mathrm{INO}_{3}\left(\mathrm{M}^{+}\right)$: calcd. 435.0331, found 435.0331.

trans-N-Methyl-3-iodo-4-phenyl-6-methyl-1-azaspiro[4,5]-deca-3,6,9-trienyl 8-acetate (2e): White solid, mp 125.8-127.4 ${ }^{\circ} \mathrm{C}$ (uncorrected); ${ }^{1} \mathrm{H}$ NMR (400 MHz) $\delta: 7.53$ (d, $\left.J=7.6 \mathrm{~Hz}, 2 \mathrm{H}\right)$, 7.41-7.32 (m, 3H), 6.24 (d, $J=10 \mathrm{~Hz}, 1 \mathrm{H}), 5.99$ (s, 1H), 5.66 (s, 1H), 5.60 (d, $J=10.0 \mathrm{~Hz}, 1 \mathrm{H}), 2.78$ (s, 3H), 1.98 (s,

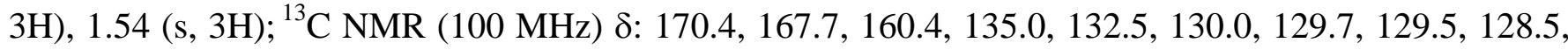
128.2, 127.1, 96.2, 71.4, 64.5, 25.9, 20.9, 16.9; IR (KBr, $\left.\mathrm{cm}^{-1}\right): 1724,1671,1239$; LRMS (EI, 70 eV) m/z (\%): 435 (M+, 15), 393 (40), 308 (100), 1291 (77); HRMS (EI) for $\mathrm{C}_{19} \mathrm{H}_{18} \mathrm{INO}_{3}\left(\mathrm{M}^{+}\right)$: calcd. 435.0331, found 435.0331 .

cis-N-Methyl-3-iodo-4-phenyl-7-methyl-1-azaspiro[4,5]-deca-3,6,9-trienyl 8-acetate (2f): White

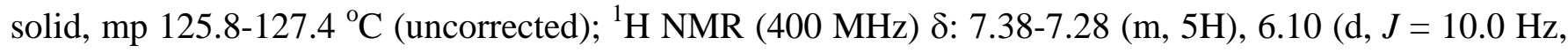
1H), 5.54 (d, $J=10.0 \mathrm{~Hz}, 1 \mathrm{H}), 5.31$ (s, 1H), 5.28 (s, 1H), 2.91 (s, 3H), 2.11 (s, 3H), 1.66 (s, 3H); ${ }^{13} \mathrm{C}$ NMR (100 MHz) $\delta: 170.6,167.1,162.7,138.2,133.2,130.3,129.5,128.4,127.8,127.6,122.9,96.3$, 70.2, 66.0, 26.5, 20.9, 19.7; IR (KBr, $\left.\mathrm{cm}^{-1}\right):$ 1705, 1653, 1239; LRMS (EI, $\left.70 \mathrm{eV}\right) \mathrm{m} / \mathrm{z}(\%): 435\left(\mathrm{M}^{+}, 1\right)$, 393 (4), 308 (38), 238 (100), 129 (75); HRMS (EI) for $\mathrm{C}_{19} \mathrm{H}_{18} \mathrm{INO}_{3}\left(\mathrm{M}^{+}\right.$): calcd. 435.0331, found 435.0330.

trans-N-Methyl-3-iodo-4-phenyl-7-methyl-1-azaspiro[4,5]-deca-3,6,9-trienyl 8-acetate (2f): White

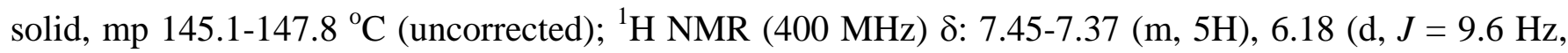
1H), 5.60-5.57 (m, 2H), 5.35 (s, 1H), 2.82 (s, 3H), 1.99 (s, 3H), 1.63 (s, 3H); ${ }^{13} \mathrm{C}$ NMR (100 MHz) $\delta:$ 170.7, 167.2, 161.4, 138.3, 132.9, 130.0, 129.5, 129.0, 128.3, 128.1, 124.5, 95.9, 70.2, 65.9, 26.2, 20.8, 
19.8; IR (KBr, $\mathrm{cm}^{-1}$ ): 1711, 1683, 1241; LRMS (EI, $70 \mathrm{eV)} \mathrm{m/z} \mathrm{( \% ):} 435$ (M+, 12), 393 (11), 308 (100), 238 (66), 129 (93); HRMS (EI) for $\mathrm{C}_{19} \mathrm{H}_{18} \mathrm{INO}_{3}\left(\mathrm{M}^{+}\right)$: calcd. 435.0331, found 435.0331.

cis-N-Methyl-3-iodo-4-phenyl-6,9-dimethyl-1-azaspiro[4,5]-deca-3,6,9-trienyl $\quad$ 8-acetate $\quad(2 \mathrm{~g})$ : White solid, mp 156.0-159.4 ${ }^{\circ} \mathrm{C}$ (uncorrected); ${ }^{1} \mathrm{H}$ NMR (400 MHz) 8: 7.34-7.28 (m, 5H), 5.81 (s, 1H), 5.31 (s, 1H), 5.24 (s, 1H), 2.80 (s, 3H), 2.07 (s, 3H), 1.74 (s, 3H), 1.48 (s, 3H); ${ }^{13} \mathrm{C}$ NMR (100 MHz) $\delta:$ 170.6, 167.5, 161.7, 137.7, 133.0, 132.8, 129.6, 128.5, 127.5, 127.2, 123.6, 96.1, 72.6, 67.3, 26.1, 20.9, 19.3, 16.6; IR (KBr, $\mathrm{cm}^{-1}$ ): 1732, 1683, 1225; LRMS (EI, $70 \mathrm{eV)} \mathrm{m/z} \mathrm{( \% ):} 449$ (M+1 17), 407 (49), 322 (100), 129 (80); HRMS (EI) for $\mathrm{C}_{20} \mathrm{H}_{20} \mathrm{INO}_{3}\left(\mathrm{M}^{+}\right)$: calcd. 449.0488, found 449.0487.

trans-N-Methyl-3-iodo-4-phenyl-6,9-dimethyl-1-azaspiro[4,5]-deca-3,6,9-trienyl 8-acetate (2g): White solid, mp 131.4-134.6 ${ }^{\circ} \mathrm{C}$ (uncorrected); ${ }^{1} \mathrm{H}$ NMR (400 MHz) $\delta: 7.47$ (d, $\left.J=7.6 \mathrm{~Hz}, 2 \mathrm{H}\right), 7.38-7.33$ (m, 3H), 5.92 (s, 1H), 5.59 (s, 1H), 5.29 (s, 1H), 2.74 (s, 3H), 1.97 (s, 3H), 1.78 (s, 3H), 1.47 (s, 3H); ${ }^{13} \mathrm{C}$ NMR (100 MHz) ઈ: 170.8, 167.7, 160.7, 137.7, 134.6, 132.6, 129.6, 128.1, 127.0, 125.1, 95.8, 72.6, 67.4, 25.9, 20.9, 19.5, 16.6; IR (KBr, $\left.\mathrm{cm}^{-1}\right): 1720,1674,1239$; LRMS (EI, $\left.70 \mathrm{eV}\right) \mathrm{m} / \mathrm{z}(\%): 449\left(\mathrm{M}^{+}, 16\right), 407$ (48), 322 (100), 129 (67); HRMS (EI) for $\mathrm{C}_{20} \mathrm{H}_{20} \mathrm{INO}_{3}\left(\mathrm{M}^{+}\right)$: calcd. 449.0488, found 449.0488.

cis-N-Methyl-3-iodo-4-phenyl-6,7-dimethyl-1-azaspiro[4,5]-deca-3,6,9-trienyl 8-acetate (2h): White solid, mp 162.6-165.9 ${ }^{\circ} \mathrm{C}$ (uncorrected); ${ }^{1} \mathrm{H}$ NMR (400 MHz) 8: 7.37-7.30 (m, 5H), 5.83 (s, 1H), 5.32 (s, 1H), 5.25 (s, 1H), 2.82 (s, 3H), 2.08 (s, 3H), 1.75 (s, 3H), 1.24 (s, 3H); ${ }^{13} \mathrm{C}$ NMR (100 MHz) $\delta:$ 170.6, 167.5, 161.7, 137.7, 133.0, 132.8, 129.6, 128.5, 127.5, 127.2, 123.6, 96.1, 72.6, 67.3, 26.1, 20.9, 19.3, 16.6; IR (KBr, $\mathrm{cm}^{-1}$ ): 1734, 1689, 1237; LRMS (EI, $\left.70 \mathrm{eV}\right)$ m/z (\%): 449 (M+, 20), 407 (55), 322 (100), 129 (84); HRMS (EI) for $\mathrm{C}_{20} \mathrm{H}_{20} \mathrm{INO}_{3}\left(\mathrm{M}^{+}\right)$: calcd. 449.0488, found 449.0488.

trans-N-Methyl-3-iodo-4-phenyl-6,7-dimethyl-1-azaspiro[4,5]-deca-3,6,9-trienyl 8-acetate (2h): White solid, mp 177.2-179.9 ${ }^{\circ} \mathrm{C}$ (uncorrected); ${ }^{1} \mathrm{H}$ NMR (400 MHz) $\delta: 7.48$ (d, $\left.J=7.6 \mathrm{~Hz}, 2 \mathrm{H}\right)$, 7.39-7.34 (m, 3H), 5.93 (s, 1H), 5.60 (s, 1H), 5.30 (s, 1H), 2.76 (s, 3H), 1.98 (s, 3H), 1.80 (s, 3H), 1.49 (s, 3H); ${ }^{13} \mathrm{C}$

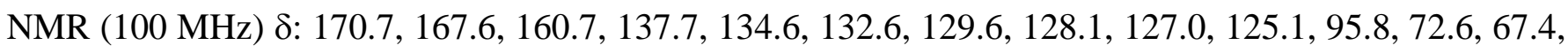


25.9, 20.9, 19.5, 16.6; IR (KBr, $\left.\mathrm{cm}^{-1}\right):$ 1700, 1683, 1235; LRMS (EI, $\left.70 \mathrm{eV}\right) \mathrm{m} / \mathrm{z}(\%): 449\left(\mathrm{M}^{+}, 20\right), 407$ (55), 322 (96), 129 (100); HRMS (EI) for $\mathrm{C}_{20} \mathrm{H}_{20} \mathrm{INO}_{3}\left(\mathrm{M}^{+}\right.$): calcd. 449.0488, found 449.0488.

cis-N-Methyl-3-iodo-4-phenyl-6-chloro-1-azaspiro[4,5]-deca-3,6,9-trienyl 8-acetate (2i): White

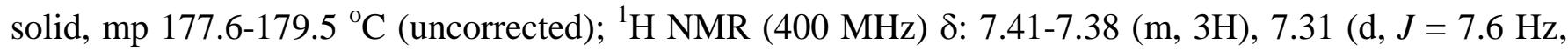
2H), $6.28(\mathrm{~d}, J=3.6 \mathrm{~Hz}, 1 \mathrm{H}), 6.15(\mathrm{~d}, J=10.0 \mathrm{~Hz}, 1 \mathrm{H}), 5.64(\mathrm{~d}, J=10.0 \mathrm{~Hz}, 1 \mathrm{H}), 5.43(\mathrm{t}, J=1.5 \mathrm{~Hz}$,

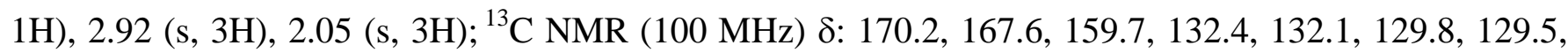
128.9, 128.6, 127.8, 127.6, 98.2, 71.8, 65.2, 26.2, 20.9; IR (KBr, $\left.\mathrm{cm}^{-1}\right)$ : 1733, 1684, 1225; LRMS (EI, 70 eV) m/z (\%): $457\left(\mathrm{M}^{+}+\right.$2, 5), $455\left(\mathrm{M}^{+}, 14\right), 413$ (-Cl, 29), 328 (47), 129 (100); HRMS (EI) for $\mathrm{C}_{18} \mathrm{H}_{15} \mathrm{ClINO}_{3}\left(\mathrm{M}^{+}\right)$: calcd. 454.9785, found 454.9785 .

trans-N-Methyl-3-iodo-4-phenyl-6-chloro-1-azaspiro[4,5]-deca-3,6,9-trienyl 8-acetate (2i): White

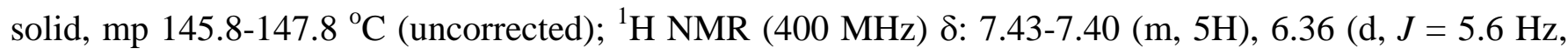
1H), 6.21 (d, $J=10.0 \mathrm{~Hz}, 1 \mathrm{H}), 5.73-5.67$ (m, 2H), 2.85 (s, 3H), 1.95 (s, 3H); ${ }^{13} \mathrm{C}$ NMR (100 MHz) $\delta:$ 170.2, 167.6, 159.1, 133.6, 132.1, 129.7, 129.2 (2C), 129.0, 128.3 (2C), 98.0, 71.8, 65.0, 26.0, 20.7; IR $\left(\mathrm{KBr}, \mathrm{cm}^{-1}\right):$ 1733, 1684, 1255; LRMS (EI, $\left.70 \mathrm{eV}\right) \mathrm{m} / \mathrm{z}(\%): 457\left(\mathrm{M}^{+}+2,7\right), 455\left(\mathrm{M}^{+}, 21\right), 413$ (29), 328 (57), 129 (100); HRMS (EI) for $\mathrm{C}_{18} \mathrm{H}_{15} \mathrm{ClINO}_{3}\left(\mathrm{M}^{+}\right)$: calcd. 454.9785, found 454.9785.

cis-N-Methyl-3-iodo-4-phenyl-6-bromo-1-azaspiro[4,5]-deca-3,6,9-trienyl 8-acetate (2j): White

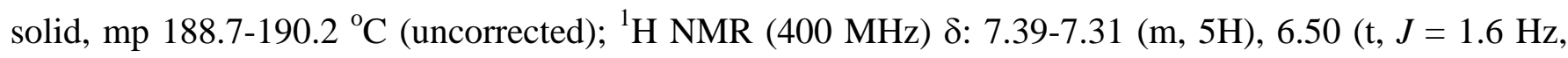
1H), $6.16(\mathrm{~d}, J=10.0 \mathrm{~Hz}, 1 \mathrm{H}), 5.67$ (d, $J=10.0 \mathrm{~Hz}, 1 \mathrm{H}), 5.30$ (s, 1H), $2.90(\mathrm{~s}, 3 \mathrm{H}), 2.07$ (s, $3 \mathrm{H}) ;{ }^{13} \mathrm{C}$ NMR (100 MHz) $\delta: 170.0,167.4,160.1,133.1,132.4,129.5,128.8,128.6,127.9,127.3,124.0,98.3$, 72.3, 65.5, 26.2, 20.8; IR (KBr, $\left.\mathrm{cm}^{-1}\right)$ : 1729, 1697, 1228; LRMS (EI, $\left.70 \mathrm{eV}\right) \mathrm{m} / \mathrm{z}(\%): 501\left(\mathrm{M}^{+}+2,13\right)$, 499 (M+, 14), 459 (24), 378 (36), 374 (44), 129 (100); HRMS (EI) for $\mathrm{C}_{18} \mathrm{H}_{15} \mathrm{BrINO}_{3}\left(\mathrm{M}^{+}\right)$: calcd. 498.9280, found 498.9278 .

trans-N-Methyl-3-iodo-4-phenyl-6-bromo-1-azaspiro[4,5]-deca-3,6,9-trienyl 8-acetate (2j): White

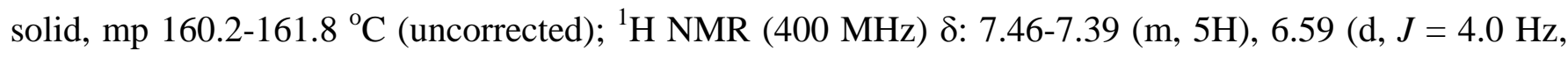


1H), 6.24 (d, $J=9.6 \mathrm{~Hz}, 1 \mathrm{H}), 5.74$ (d, $J=10.0 \mathrm{~Hz}, 1 \mathrm{H}), 5.63$ (t, $J=3.6 \mathrm{~Hz}, 1 \mathrm{H}), 2.84$ (s, $3 \mathrm{H}), 1.94$ (s,

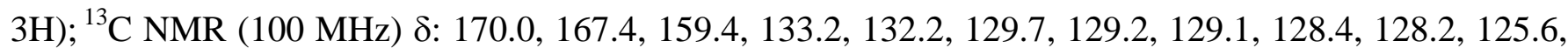
98.1, 72.3, 65.2, 26.0, 20.6; IR (KBr, $\left.\mathrm{cm}^{-1}\right)$ : 1730, 1693, 1229; LRMS (EI, 70 eV) m/z (\%): $501\left(\mathrm{M}^{+}+2\right.$, 21), 499 (M+, 20), 459 (23), 378 (27), 374 (42), 129 (100); HRMS (EI) for $\mathrm{C}_{18} \mathrm{H}_{15} \mathrm{BrINO}_{3}\left(\mathrm{M}^{+}\right.$): calcd. 498.9280, found 498.9280 .

cis-N-Methyl-3,6-diiodo-4-phenyl-1-azaspiro[4,5]-deca-3,6,9-trienyl 8-acetate (2k): White solid, mp

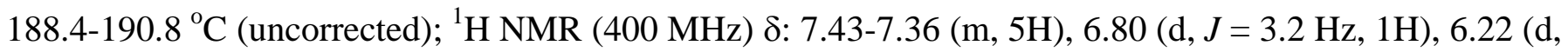
$J=10.0 \mathrm{~Hz}, 1 \mathrm{H}), 5.75$ (d, $J=9.6 \mathrm{~Hz}, 1 \mathrm{H}), 5.21$ (d, $J=4.4 \mathrm{~Hz}, 1 \mathrm{H}), 2.91$ (s, 3H), 2.09 (s, 3H); ${ }^{13} \mathrm{C}$ NMR

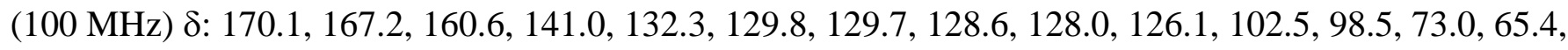
26.3, 20.9; IR (KBr, $\mathrm{cm}^{-1}$ ): 1740, 1697, 1230; LRMS (EI, $70 \mathrm{eV)} \mathrm{m/z} \mathrm{( \% ):} 547$ (M+1 15), 505 (16), 420 (81), 378 (100), 251 (92); HRMS (EI) for $\mathrm{C}_{18} \mathrm{H}_{15} \mathrm{I}_{2} \mathrm{NO}_{3}\left(\mathrm{M}^{+}\right)$: calcd. 546.9141, found 546.9141.

trans-N-Methyl-3,6-diiodo-4-phenyl-1-azaspiro[4,5]-deca-3,6,9-trienyl 8-acetate (2k): White solid,

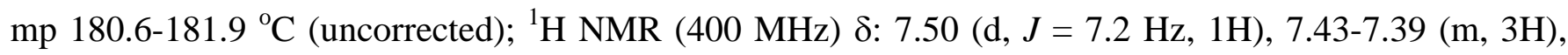
6.90 (d, $J=4.2 \mathrm{~Hz}, 1 \mathrm{H}), 6.30$ (d, $J=9.6 \mathrm{~Hz}, 1 \mathrm{H}), 5.82$ (d, $J=9.6 \mathrm{~Hz}, 1 \mathrm{H}), 5.51$ (t, $J=3.6 \mathrm{~Hz}, 1 \mathrm{H}), 2.84$ (s, 3H), 1.96 (s, 3H); IR (KBr, cm ${ }^{-1}$ ): 1728, 1690, 1228; LRMS (EI, $\left.70 \mathrm{eV}\right) \mathrm{m} / \mathrm{z}$ (\%): 547 (M+, 30$), 505$ (12), 420 (79), 378 (91), 251 (81); HRMS (EI) for $\mathrm{C}_{18} \mathrm{H}_{15} \mathrm{I}_{2} \mathrm{NO}_{3}\left(\mathrm{M}^{+}\right.$): calcd. 546.9141, found 546.9140.

cis-N-methyl-3-iodo-4-(p-methylphenyl)-1-azaspiro[4,5]-deca-3,6,9-trienyl 8-acetate (2m): White

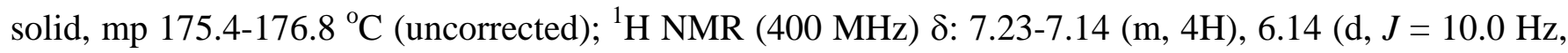
2H), 5.57 (d, $J=10.0 \mathrm{~Hz}, 1 \mathrm{H}), 5.32$ (s, 1H), 2.91 (s, 3H), 2.34 (s, 3H), 2.00 (s, 3H); ${ }^{13} \mathrm{C}$ NMR (100 MHz) $\delta: 170.4,167.3,162.1,139.7,130.2,129.4,129.2,128.1,127.7,96.1,69.0,63.4,26.5,21.4,21.0$; IR (KBr, $\left.\mathrm{cm}^{-1}\right):$ 1736, 1691, 1223; LRMS (EI, $\left.70 \mathrm{eV}\right)$ m/z (\%): $435\left(\mathrm{M}^{+}, 8\right), 308$ (71), 393 (6), 143 (100); HRMS (EI) for $\mathrm{C}_{19} \mathrm{H}_{18} \mathrm{INO}_{3}\left(\mathrm{M}^{+}\right)$: calcd. 435.0331, found 435.0331.

trans-N-methyl-3-iodo-4-(p-methylphenyl)-1-azaspiro[4,5]-deca-3,6,9-trienyl 8-acetate (2m): White solid, mp 161.4-162.3 ${ }^{\circ} \mathrm{C}$ (uncorrected); ${ }^{1} \mathrm{H}$ NMR (400 MHz) $\delta: 7.42$ (d, $\left.J=8.0 \mathrm{~Hz}, 2 \mathrm{H}\right), 7.18$ (d, $J$ 
$=8.0 \mathrm{~Hz}, 2 \mathrm{H}), 6.24(\mathrm{~d}, J=10.0 \mathrm{~Hz}, 1 \mathrm{H}), 5.64-5.61(\mathrm{~m}, 3 \mathrm{H}), 2.82(\mathrm{~s}, 3 \mathrm{H}), 2.36(\mathrm{~s}, 3 \mathrm{H}), 2.00(\mathrm{~s}, 3 \mathrm{H}) ;{ }^{13} \mathrm{C}$

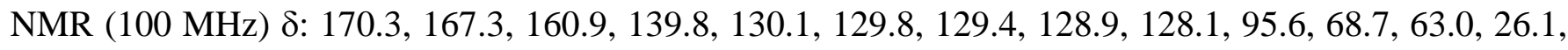
21.4, 20.9; IR (KBr, $\left.\mathrm{cm}^{-1}\right):$ 1723, 1701, 1242; LRMS (EI, $\left.70 \mathrm{eV}\right) \mathrm{m} / \mathrm{z}(\%): 435\left(\mathrm{M}^{+}, 36\right), 308$ (100); HRMS (EI) for $\mathrm{C}_{19} \mathrm{H}_{18} \mathrm{INO}_{3}\left(\mathrm{M}^{+}\right)$: calcd. 435.0331, found 435.0330 .

cis-N-methyl-3-iodo-4-(p-methoxyphenyl)-1-azaspiro[4,5]-deca-3,6,9-trienyl 8-acetate (2n): White solid, mp 170.6-172.9 ${ }^{\circ} \mathrm{C}$ (uncorrected); ${ }^{1} \mathrm{H}$ NMR (400 MHz) $\delta: 7.33$ (d, $\left.J=8.8 \mathrm{~Hz}, 2 \mathrm{H}\right), 6.87$ (d, $J=8.8$ Hz, 2H), 6.15 (d, $J=10.0 \mathrm{~Hz}, 2 \mathrm{H}), 5.56$ (d, $J=10.0 \mathrm{~Hz}, 1 \mathrm{H}), 5.36$ (s, 1H), 3.81 (s, 3H), 2.90 (s, 3H), 2.08

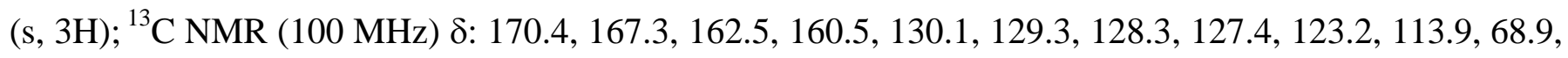
63.4, 55.3, 26.4, 21.0; IR (KBr, $\left.\mathrm{cm}^{-1}\right)$ : 1733, 1684, 1259; LRMS (EI, $\left.70 \mathrm{eV}\right) \mathrm{m} / \mathrm{z}$ (\%): 451 (M $\left.^{+}, 27\right), 409$ (16), 325 (69), 159 (100); HRMS (EI) for $\mathrm{C}_{19} \mathrm{H}_{18} \mathrm{INO}_{4}\left(\mathrm{M}^{+}\right)$: calcd. 451.0281, found 451.0280 .

\section{trans-N-methyl-3-iodo-4-(p-methoxyphenyl)-1-azaspiro[4,5]-deca-3,6,9-trienyl 8-acetate $\quad(2 n)$ :}

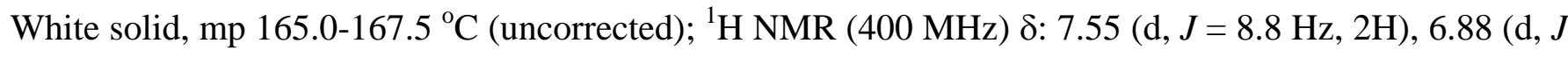
$=8.8 \mathrm{~Hz}, 2 \mathrm{H}), 6.25$ (d, $J=10.0 \mathrm{~Hz}, 1 \mathrm{H}), 5.67-5.61(\mathrm{~m}, 3 \mathrm{H}), 3.82(\mathrm{~s}, 3 \mathrm{H}), 2.81(\mathrm{~s}, 3 \mathrm{H}), 2.03(\mathrm{~s}, 3 \mathrm{H}) ;{ }^{13} \mathrm{C}$

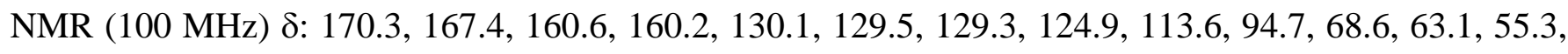
26.0, 20.9; IR (KBr, $\left.\mathrm{cm}^{-1}\right):$ 1700, 1683, 1242; LRMS (EI, $\left.70 \mathrm{eV}\right) \mathrm{m} / \mathrm{z}(\%): 451\left(\mathrm{M}^{+}, 45\right), 324$ (77), 159 (100); HRMS (EI) for $\mathrm{C}_{19} \mathrm{H}_{18} \mathrm{INO}_{4}\left(\mathrm{M}^{+}\right)$: calcd. 451.0281, found 451.0281 .

cis-N-methyl-3-iodo-4-methyl-1-azaspiro[4,5]-deca-3,6,9-trienyl 8-acetate (2p): White solid, mp 148.8-150.1 ${ }^{\circ} \mathrm{C}$ (uncorrected); ${ }^{1} \mathrm{H}$ NMR (400 MHz) $\delta: 6.22$ (d, $\left.J=10.0 \mathrm{~Hz}, 2 \mathrm{H}\right), 5.66(\mathrm{~s}, 1 \mathrm{H}), 5.41$ (d, $J=$

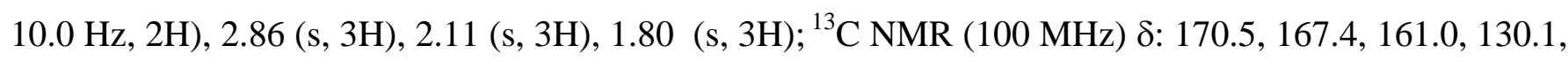
128.3, 94.4, 68.7, 63.1, 26.6, 21.0, 15.5; IR (KBr, $\left.\mathrm{cm}^{-1}\right)$ : 1711, 1684, 1236; LRMS (EI, 70 eV) m/z (\%): $359\left(\mathrm{M}^{+}, 100\right), 317$ (73), 232 (30), 190 (64); HRMS (EI) for $\mathrm{C}_{13} \mathrm{H}_{14} \mathrm{INO}_{3}\left(\mathrm{M}^{+}\right)$: calcd. 359.0018, found 359.0015.

trans-N-methyl-3-iodo-4-methyl-1-azaspiro[4,5]-deca-3,6,9-trienyl 8-acetate (2p): White solid, mp 143.6-145.5 ${ }^{\circ} \mathrm{C}$ (uncorrected); ${ }^{1} \mathrm{H}$ NMR (400 MHz) $\delta: 6.25$ (d, $\left.J=10.0 \mathrm{~Hz}, 2 \mathrm{H}\right), 5.70(\mathrm{~s}, 1 \mathrm{H}), 5.41$ (d, $J=$ 


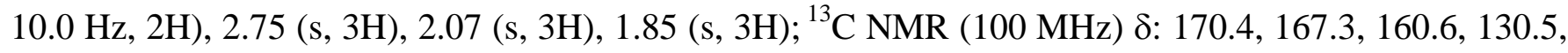
129.7, 94.4, 68.7, 63.3, 26.4, 21.0, 15.6; IR (KBr, $\left.\mathrm{cm}^{-1}\right)$ : 1700, 1650, 1235; LRMS (EI, 70 eV) m/z (\%): 359 ( $\mathrm{M}^{+}$, 100), 317 (83), 232 (43), 190 (64); HRMS (EI) for $\mathrm{C}_{13} \mathrm{H}_{14} \mathrm{INO}_{3}\left(\mathrm{M}^{+}\right.$): calcd. 359.0018, found 359.0017.

cis-3-Iodo-4-phenyl-1-oxaspiro[4,5]-deca-3,6,9-trienyl 8-acetate (2t): White solid, mp 201.2-202.8 ${ }^{\circ} \mathrm{C}$ (uncorrected); ${ }^{1} \mathrm{H}$ NMR (400 MHz) $\delta: ~ 7.45-7.34 ~(m, 5 H), 6.14(\mathrm{~d}, J=10.0 \mathrm{~Hz}, 2 \mathrm{H}), 5.82$ (d, $J=10.0$

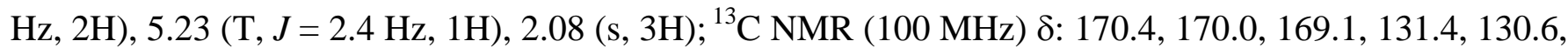
128.8, 127.3, 126.3, 86.9, 85.1, 63.3, 21.0; IR (KBr, $\left.\mathrm{cm}^{-1}\right)$ : 1760, 1722, 1235; LRMS (EI, 70 eV) m/z (\%): $408\left(\mathrm{M}^{+}, 1\right), 281$ (3), 366 (43), 129 (100); HRMS (EI) for $\mathrm{C}_{17} \mathrm{H}_{13} \mathrm{IO}_{4}\left(\mathrm{M}^{+}\right.$): calcd. 407.9859, found 407.9859.

trans-3-Iodo-4-phenyl-1-oxaspiro[4,5]-deca-3,6,9-trienyl 8-acetate (2t): White solid, mp 185.1-186.4 ${ }^{\circ} \mathrm{C}$ (uncorrected); ${ }^{1} \mathrm{H}$ NMR (400 MHz) $\delta: 7.56$ (d, $\left.J=8.4 \mathrm{~Hz}, 2 \mathrm{H}\right), 7.45-7.39$ (m, 3H), 6.25 (d, $J=11.2$ Hz, 2H), $5.88(\mathrm{~d}, J=9.0 \mathrm{~Hz}, 3 \mathrm{H}), 5.64(\mathrm{t}, J=2.4 \mathrm{~Hz}, 1 \mathrm{H}), 1.98(\mathrm{~s}, 3 \mathrm{H}) ;{ }^{13} \mathrm{C}$ NMR $(100 \mathrm{MHz}) \delta: 170.1$, 169.1, 168.4, 131.0, 130.7, 128.4, 128.0, 86.3, 85.0, 63.1, 20.8; IR (KBr, cm $\left.{ }^{-1}\right): 1770,1748,1221$; LRMS (EI, $70 \mathrm{eV}$ ) m/z (\%): 408 (M+, 3), 366 (31), 281 (11), 129 (100); HRMS (EI) for $\mathrm{C}_{17} \mathrm{H}_{13} \mathrm{IO}_{4}\left(\mathrm{M}^{+}\right)$: calcd. 407.9859, found 407.9858.

N-Methyl-3-iodo-4-phenyl-1-azaspiro[4,5]-deca-3,6,8-trien-10-one (3r): Pale yellow solid, mp

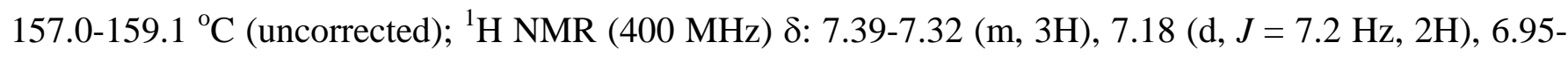
$6.90(\mathrm{~m}, 1 \mathrm{H}), 6.52-6.48(\mathrm{~m}, 1 \mathrm{H}), 6.13(\mathrm{t}, J=10.0 \mathrm{~Hz}, 2 \mathrm{H}), 2.84(\mathrm{~s}, 3 \mathrm{H}) ;{ }^{13} \mathrm{C}$ NMR $(100 \mathrm{MHz}) \delta: 194.4$, 168.8, 157.4, 141.9, 137.0, 132.1, 129.8, 128.2, 127.7, 127.6, 127.1, 97.8, 27.5; IR (KBr, $\left.\mathrm{cm}^{-1}\right): 1706$, 1667, 1236; LRMS (EI, $70 \mathrm{eV}) \mathrm{m} / \mathrm{z}(\%): 377$ (M+1 100$), 250$ (25), 129 (45); HRMS (EI) for $\mathrm{C}_{16} \mathrm{H}_{12} \mathrm{INO}_{2}$ $\left(\mathrm{M}^{+}\right)$: calcd. 376.9913, found 376.9913. 
N-Methyl-3-iodo-4-phenyl-1-azaspiro[4,5]-deca-3,6,9-trien-8-one (3u): White solid, mp 195.6-197.3

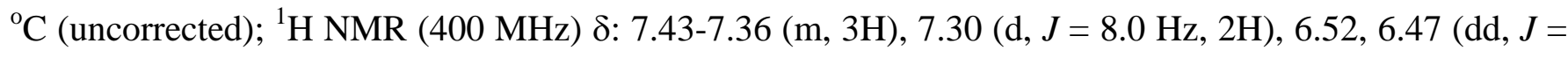

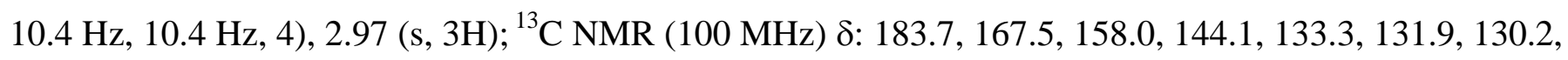
128.7, 127.9, 98.2, 70.4, 27.0; IR (KBr, $\left.\mathrm{cm}^{-1}\right)$ : 1699, 1662, 1361; LRMS (EI, $\left.70 \mathrm{eV}\right) \mathrm{m} / \mathrm{z}(\%): 377\left(\mathrm{M}^{+}\right.$, 14), 248 (94), 129 (100); HRMS (EI) for $\mathrm{C}_{16} \mathrm{H}_{12} \mathrm{INO}_{2}\left(\mathrm{M}^{+}\right)$: calcd. 376.9913, found 376.9912.

\section{(C) References}

(1) Pinto, A.; Neuville, L.; Retailleau, P.; Zhu, J. Org. Lett. 2006, 8, 4927 and references cited therein. 
(D) Spectra

cis-N-Methyl-3-iodo-4-phenyl-1-azaspiro[4,5]-deca-3,6,9-trienyl 8-acetate (2a)

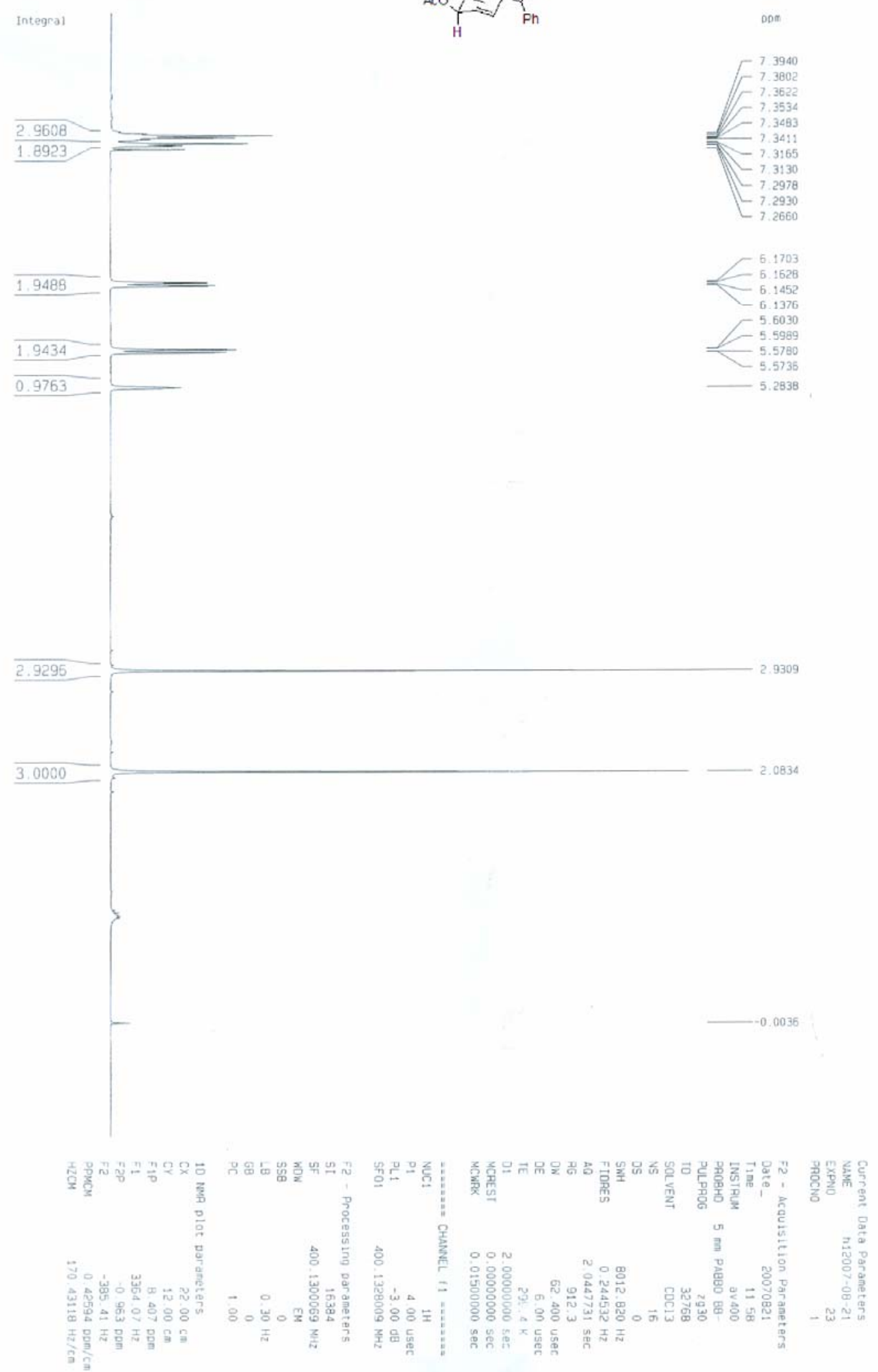


cis-N-Methyl-3-iodo-4-phenyl-1-azaspiro[4,5]-deca-3,6,9-trienyl 8-acetate (2a)

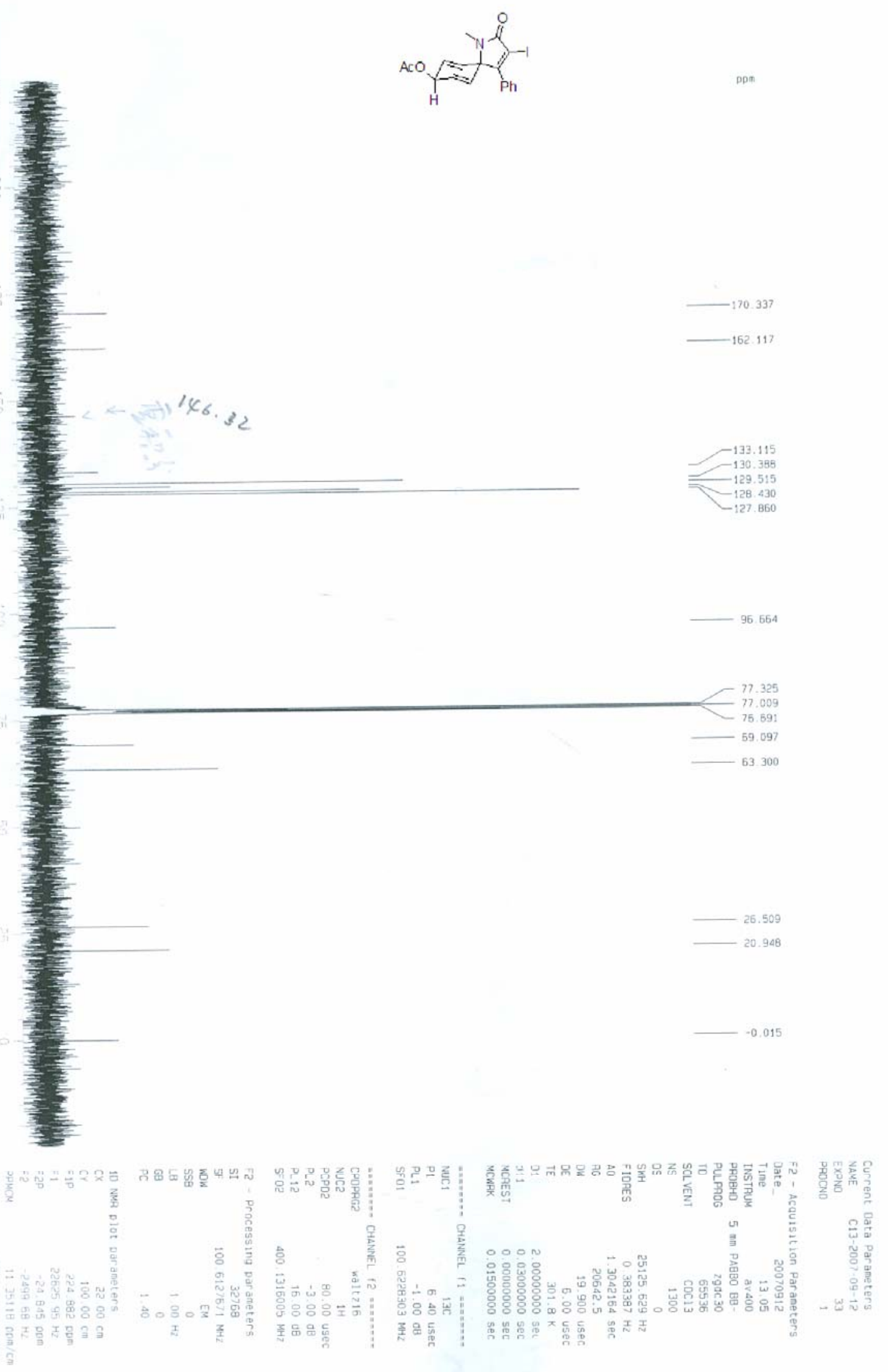


trans-N-Methyl-3-iodo-4-phenyl-1-azaspiro[4,5]-deca-3,6,9-trienyl 8-acetate (2a)

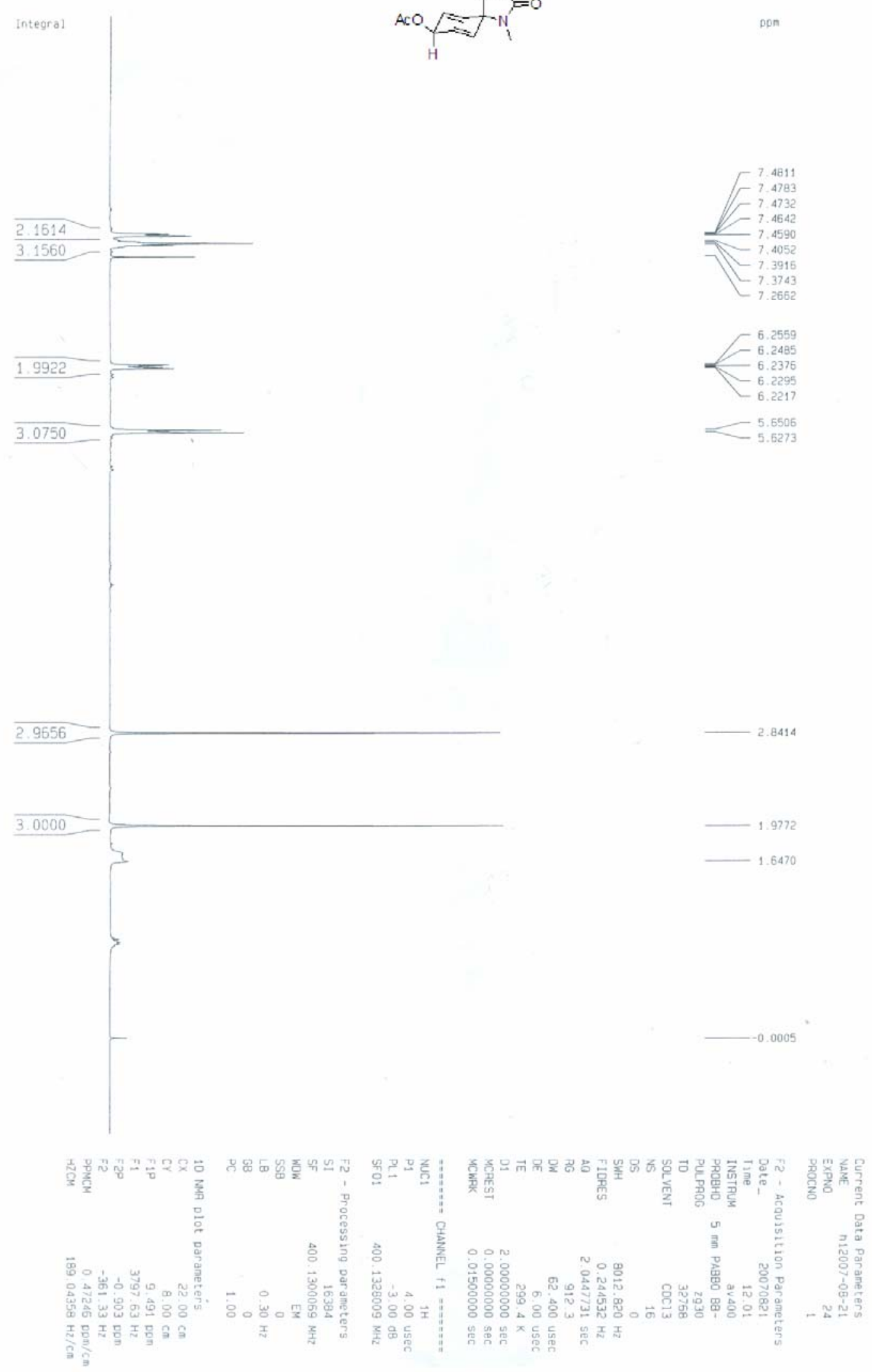


trans-N-Methyl-3-iodo-4-phenyl-1-azaspiro[4,5]-deca-3,6,9-trienyl 8-acetate (2a)

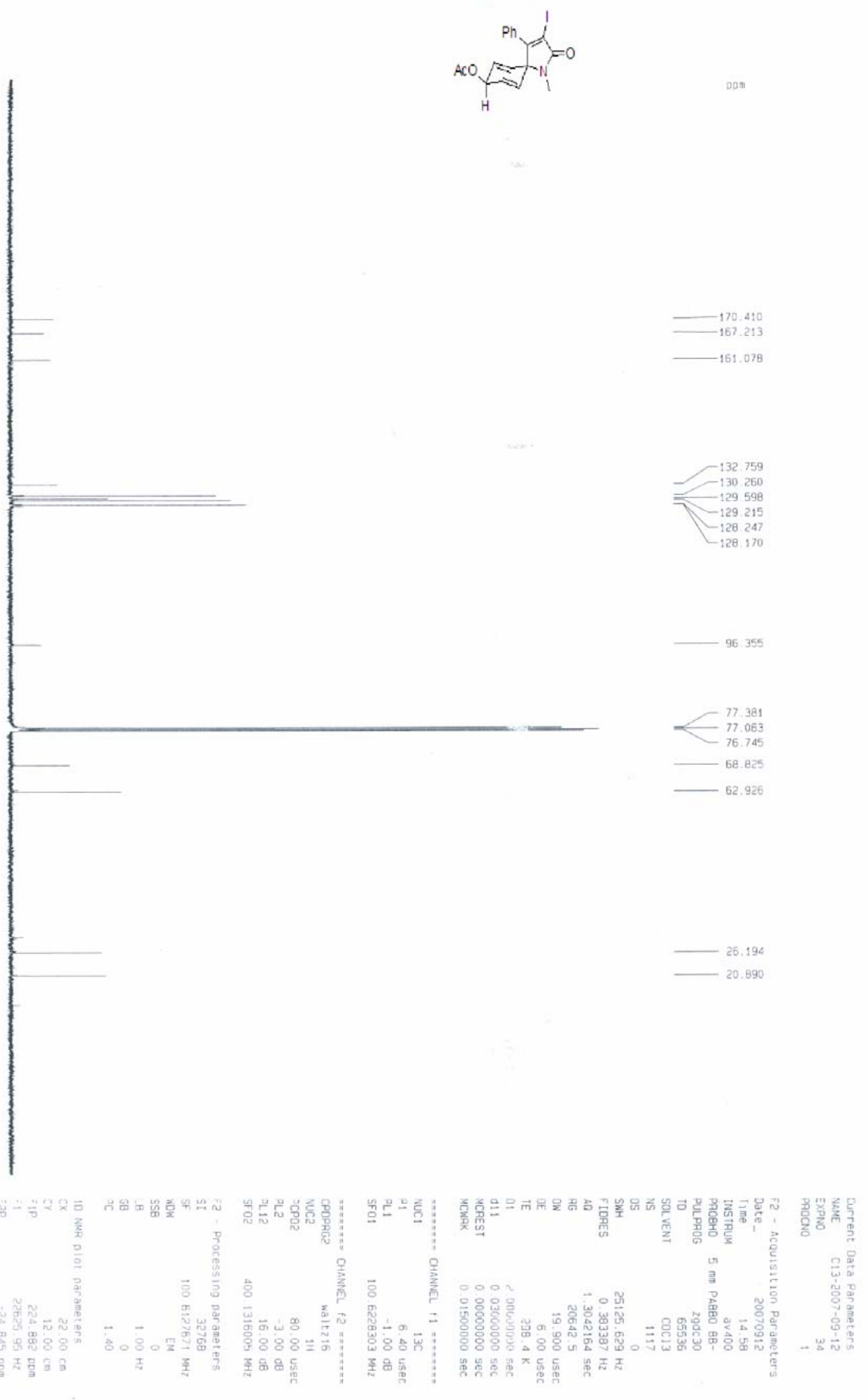


cis-N-Methyl-3-iodo-4-phenyl-6-methyl-1-azaspiro[4,5]-deca-3,6,9-trienyl 8-acetate (2e)

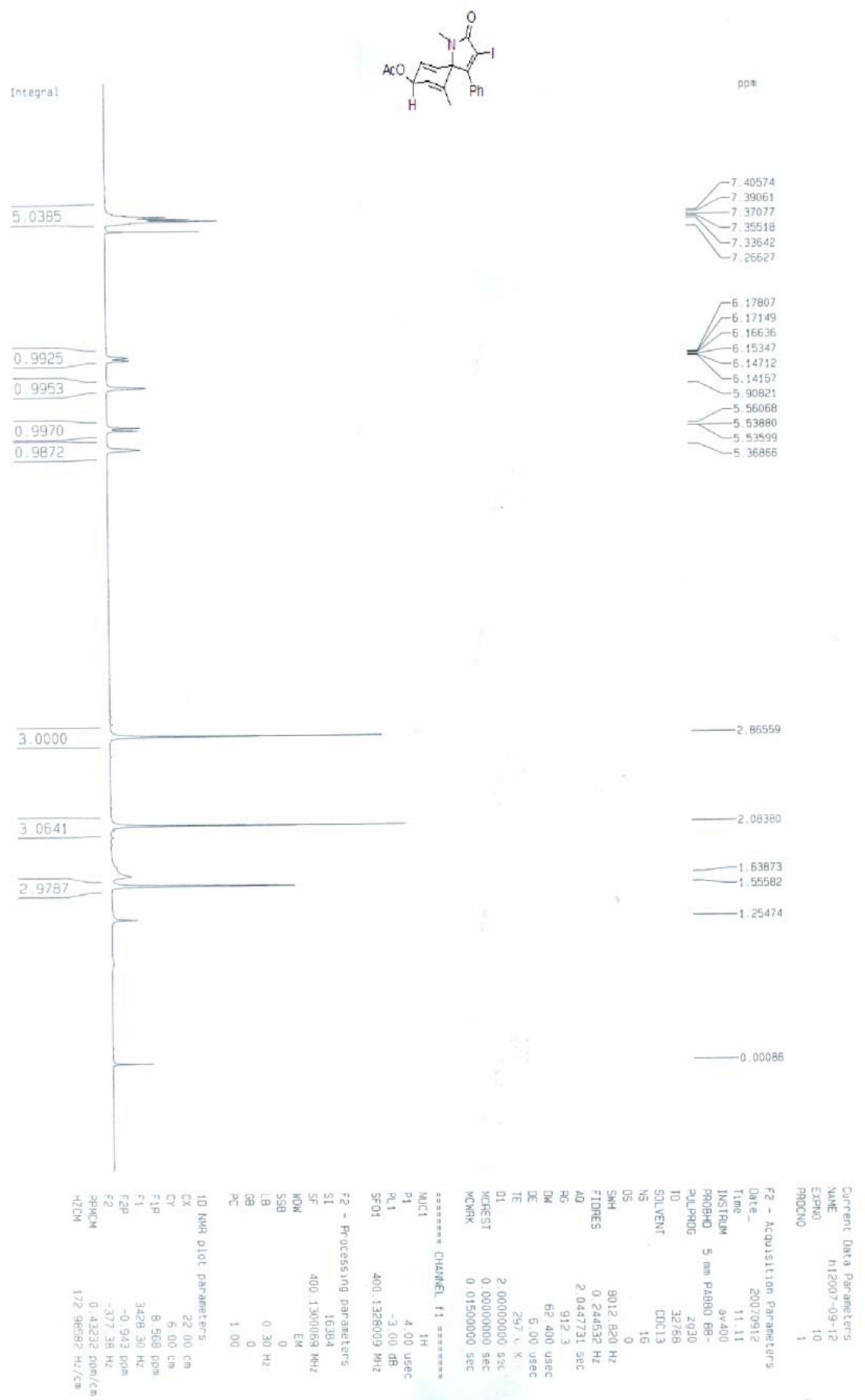


cis-N-Methyl-3-iodo-4-phenyl-6-methyl-1-azaspiro[4,5]-deca-3,6,9-trienyl 8-acetate (2e)
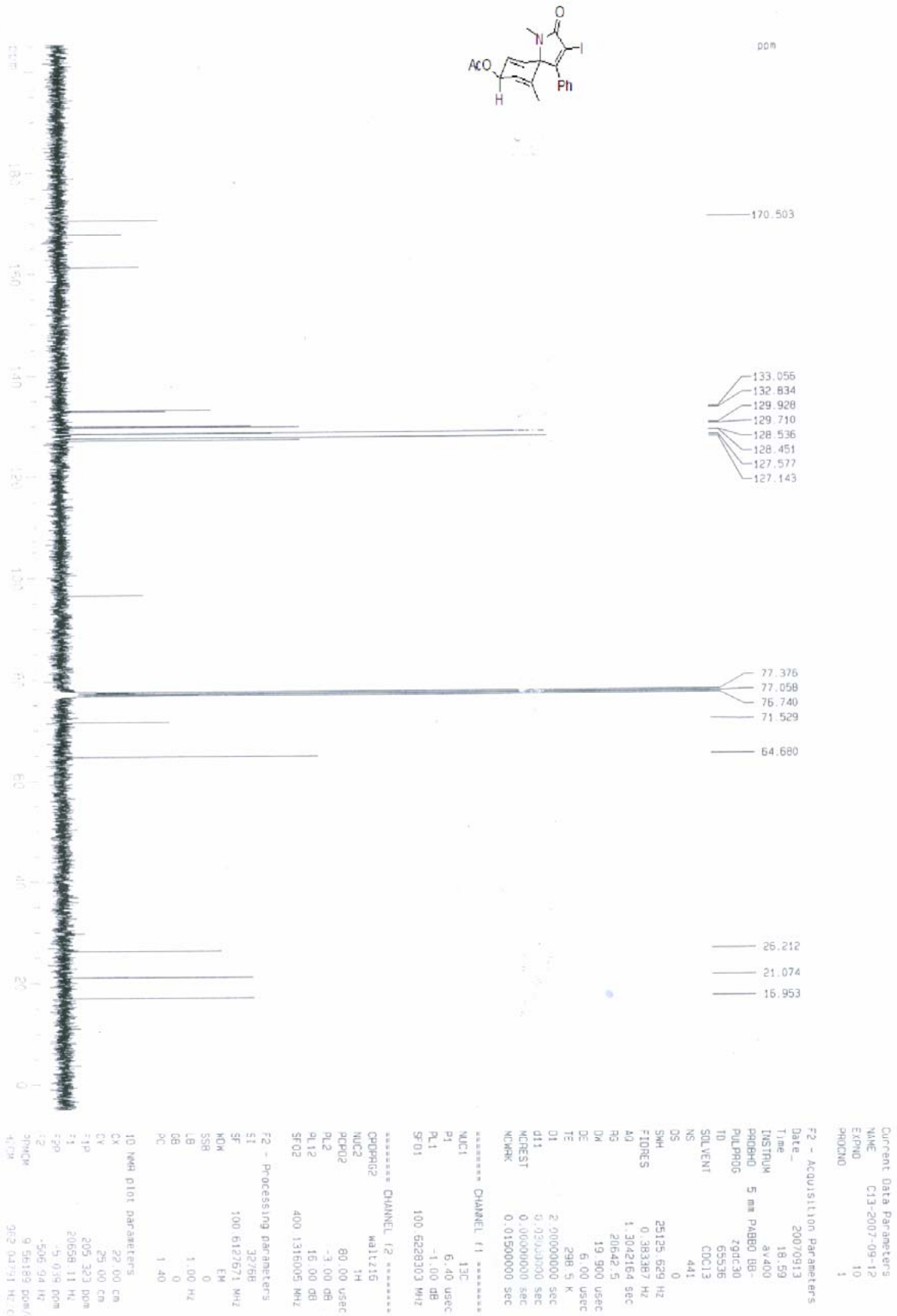
trans-N-Methyl-3-iodo-4-phenyl-6-methyl-1-azaspiro[4,5]-deca-3,6,9-trienyl 8-acetate (2e)

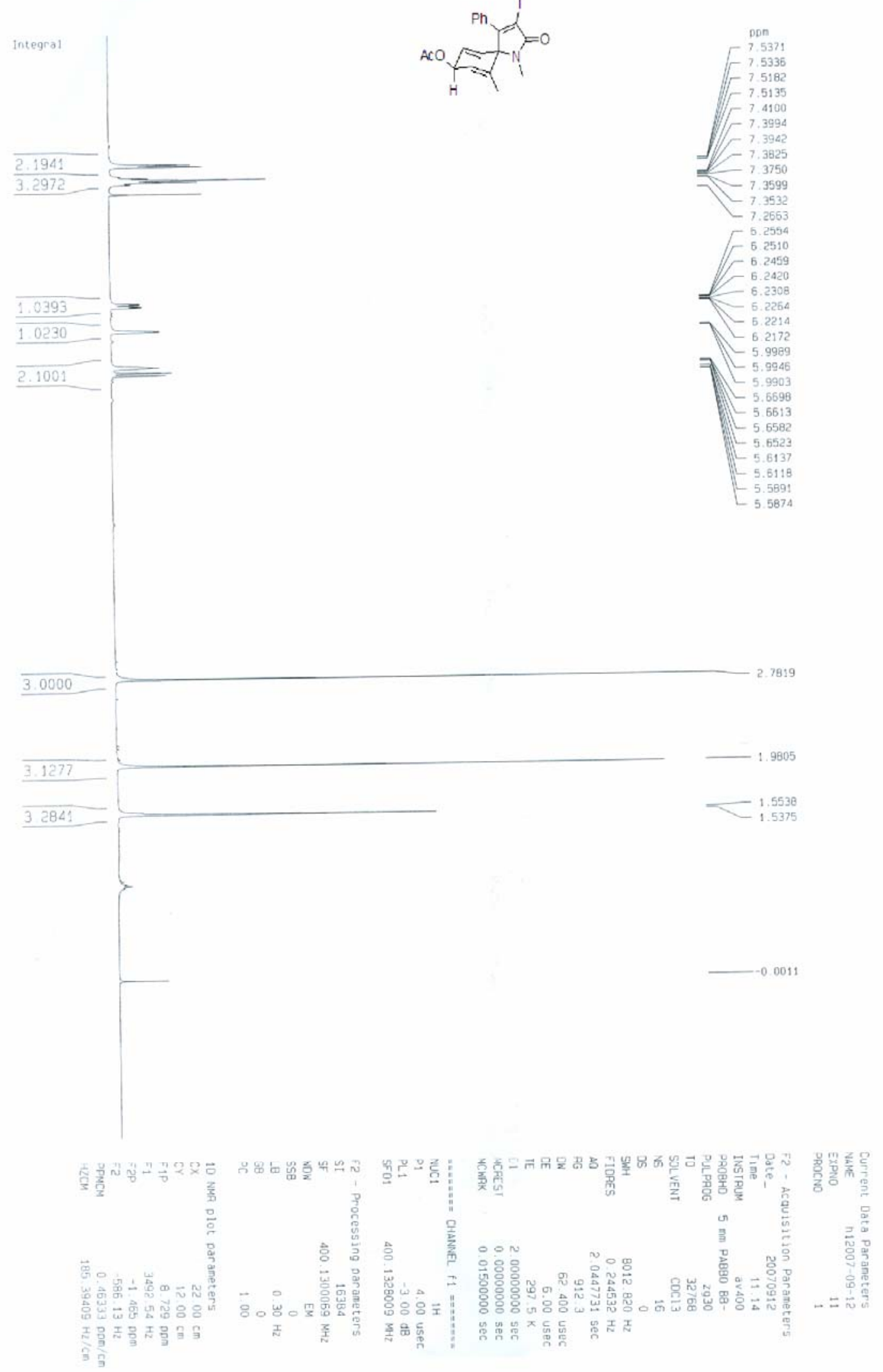


trans-N-Methyl-3-iodo-4-phenyl-6-methyl-1-azaspiro[4,5]-deca-3,6,9-trienyl 8-acetate (2e)

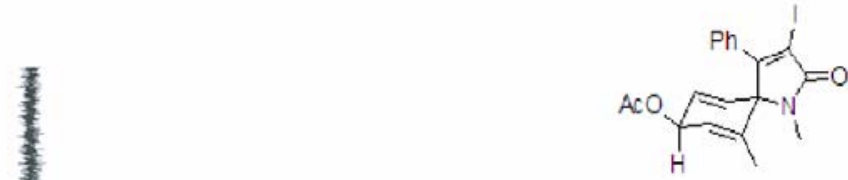

$-170.438$
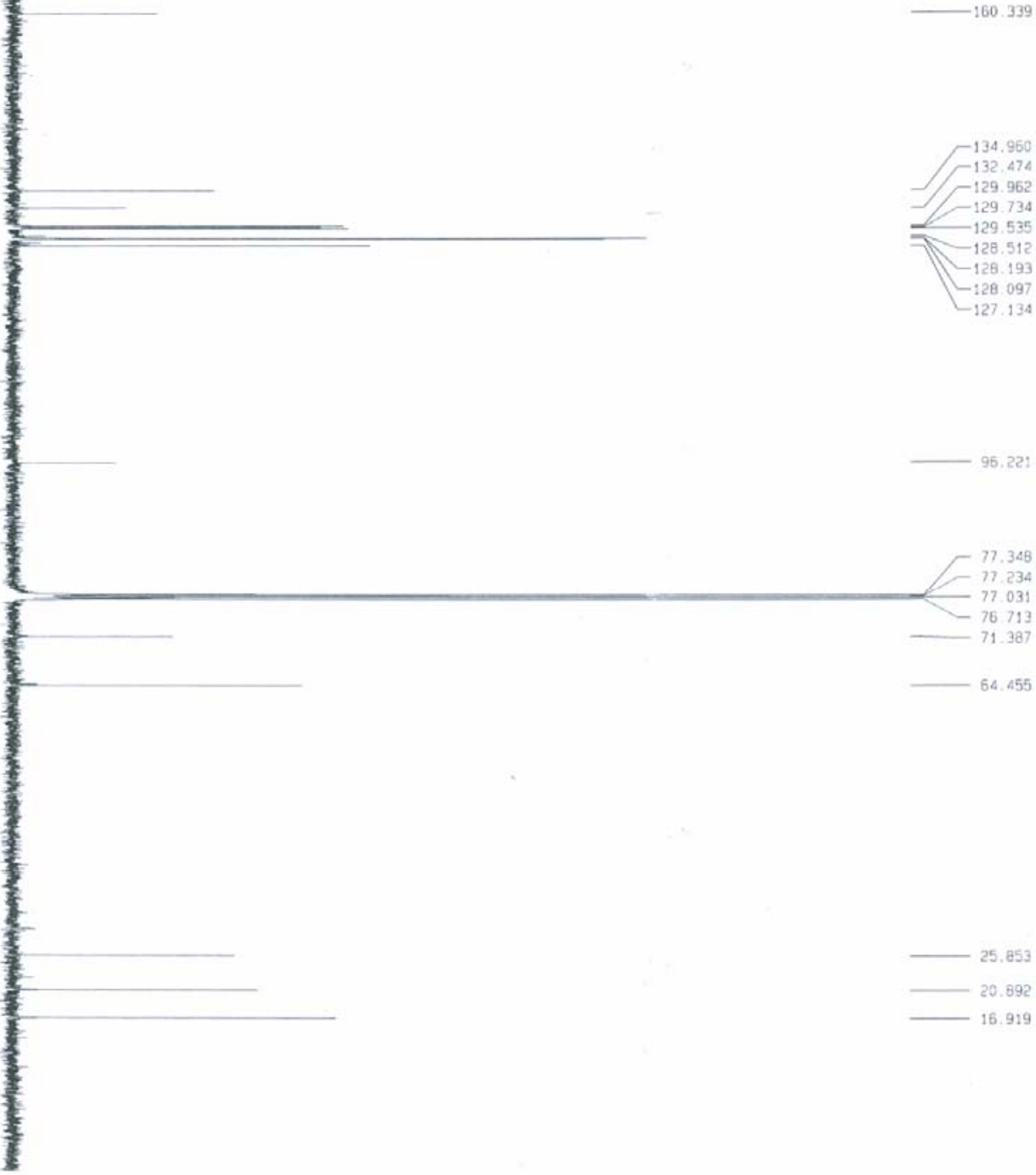

$-77.031$
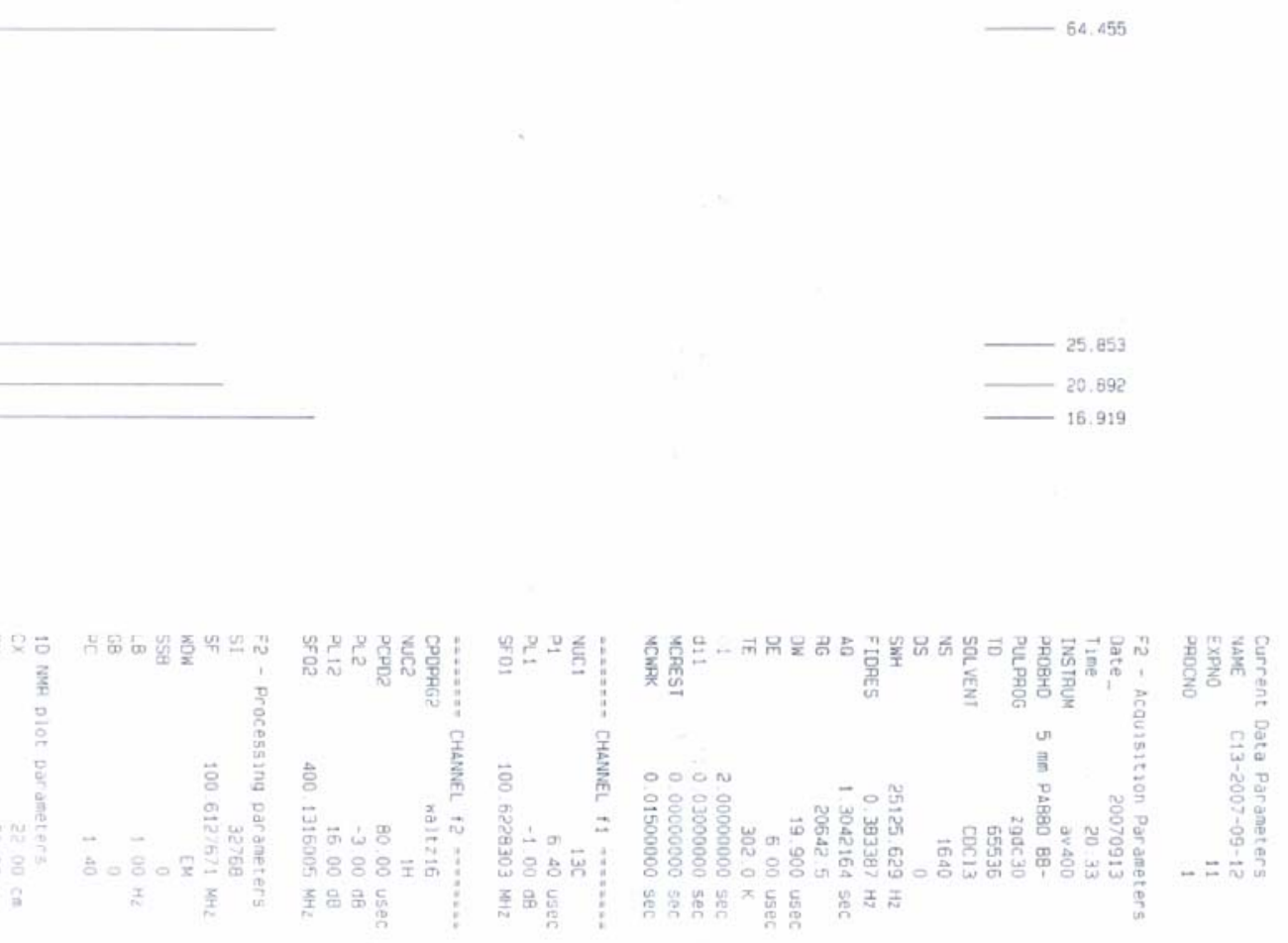
cis-N-Methyl-3-iodo-4-phenyl-7-methyl-1-azaspiro[4,5]-deca-3,6,9-trienyl 8-acetate (2f)

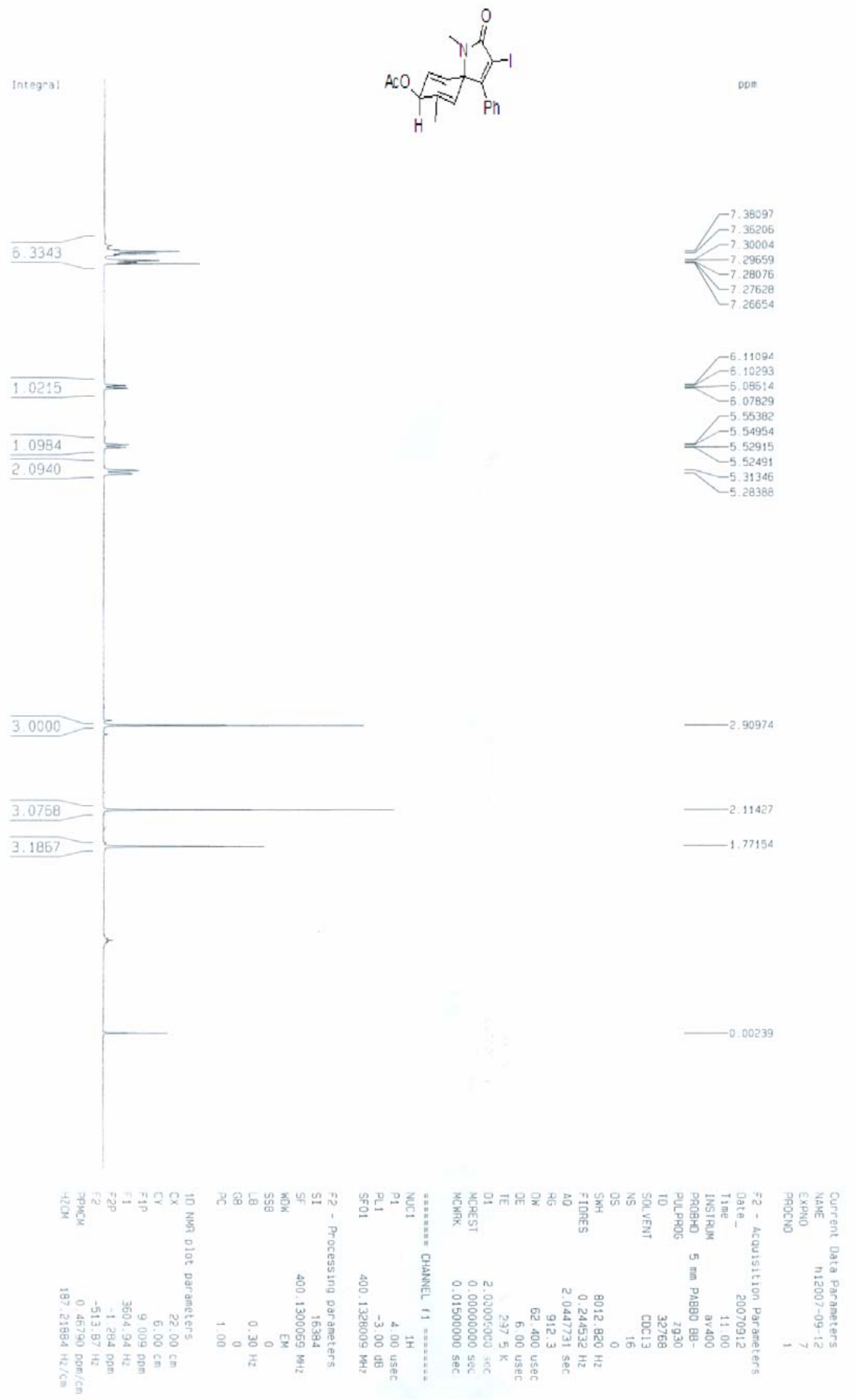


cis-N-Methyl-3-iodo-4-phenyl-6-methyl-1-azaspiro[4,5]-deca-3,6,9-trienyl 8-acetate (2f)
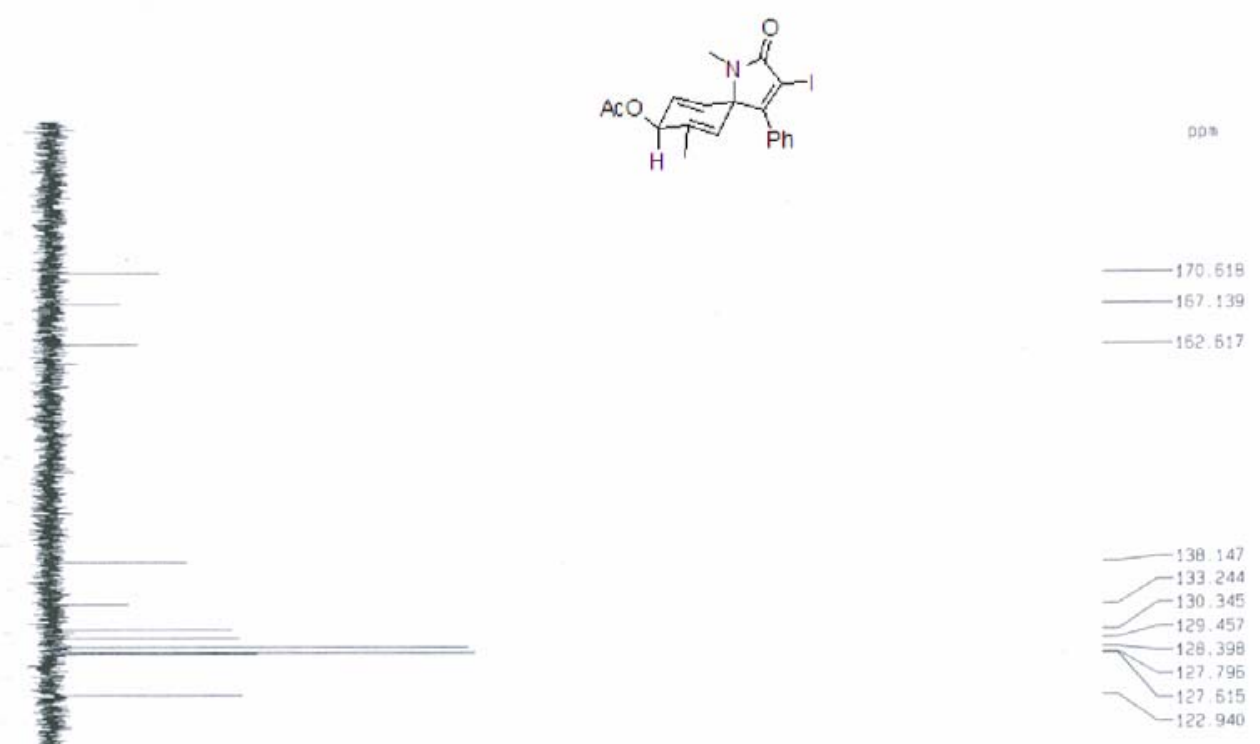

$-96.254$
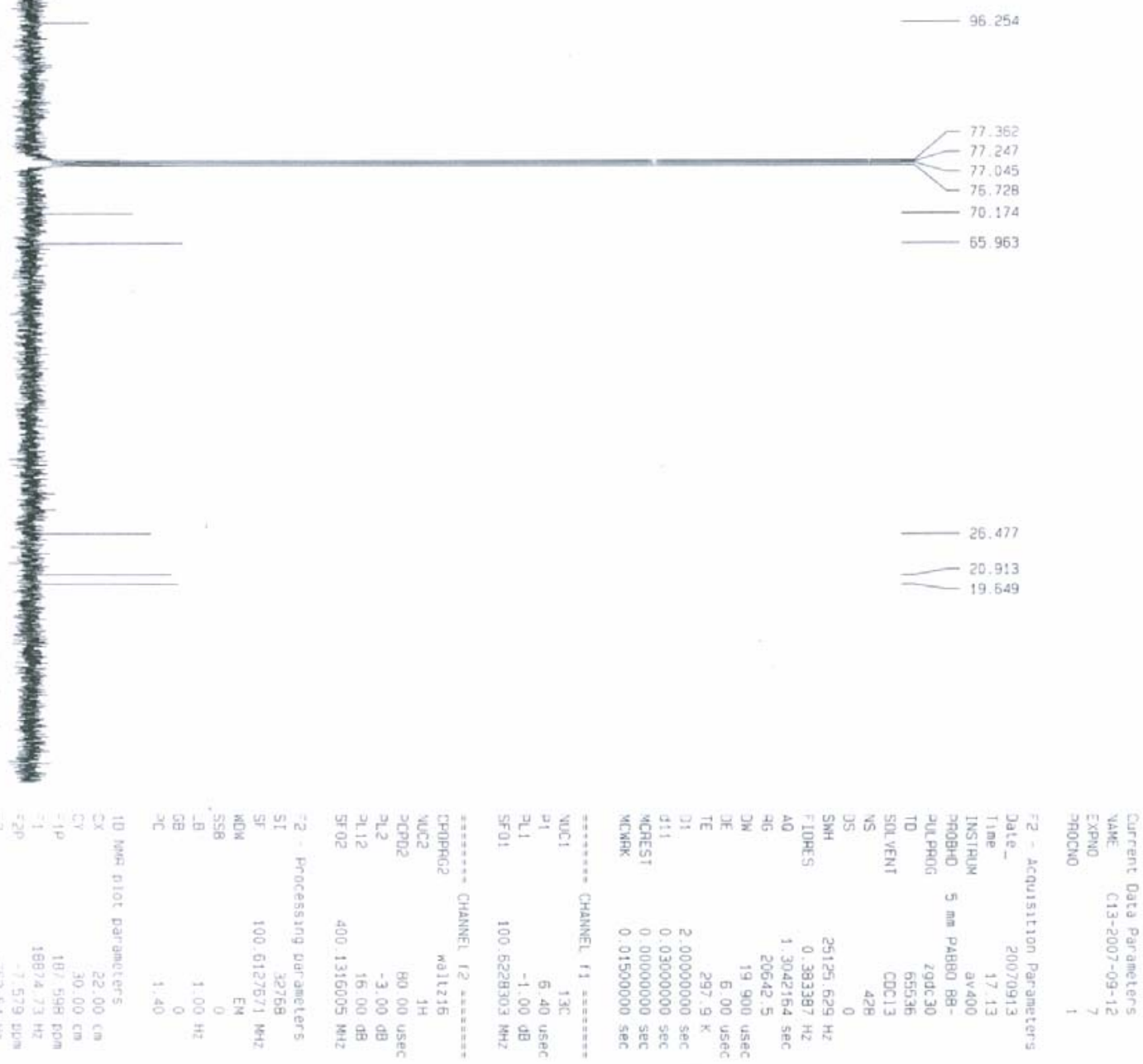
trans-N-Methyl-3-iodo-4-phenyl-6-methyl-1-azaspiro[4,5]-deca-3,6,9-trienyl 8-acetate (2f)

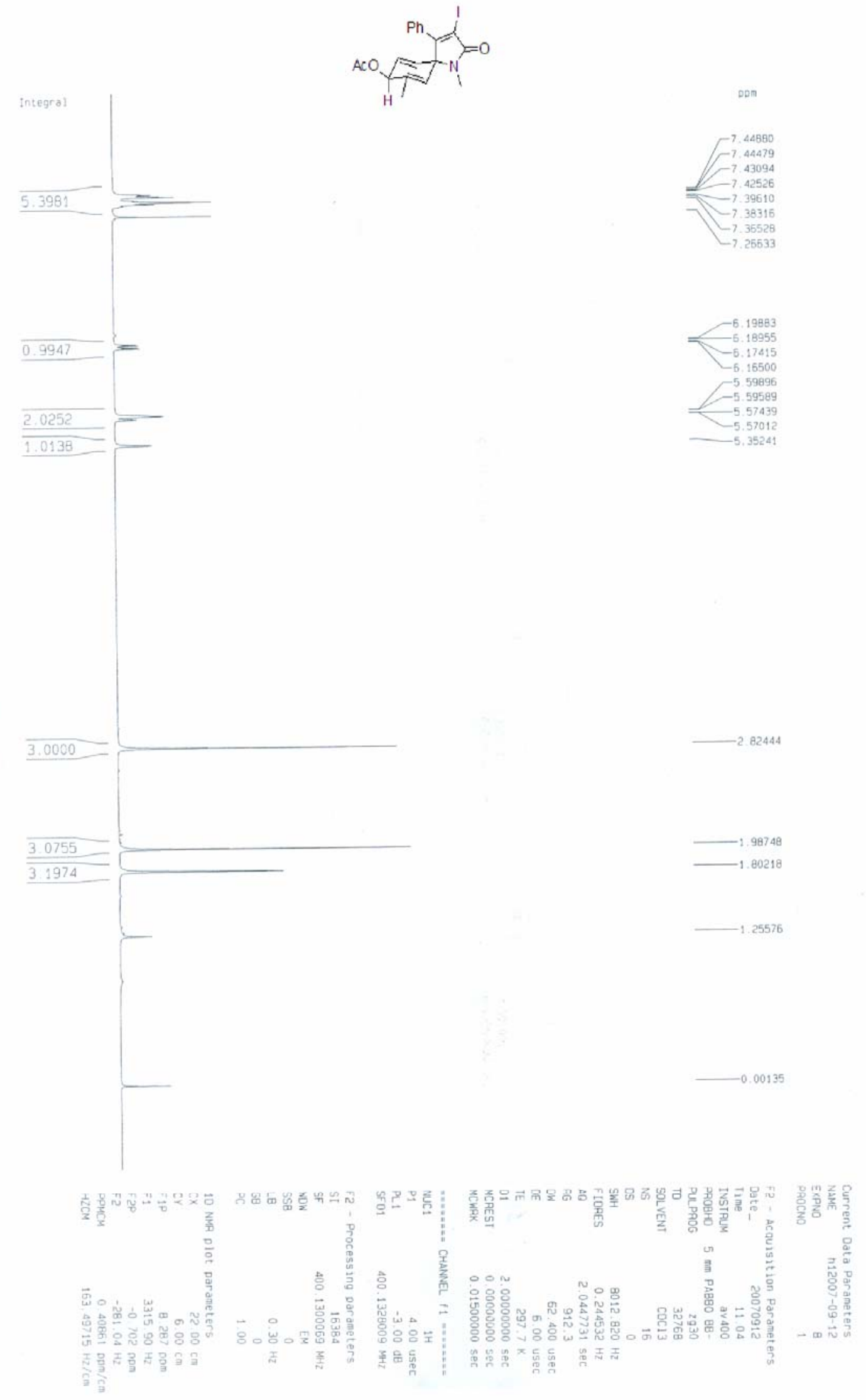


trans-N-Methyl-3-iodo-4-phenyl-6-methyl-1-azaspiro[4,5]-deca-3,6,9-trienyl 8-acetate (2f)
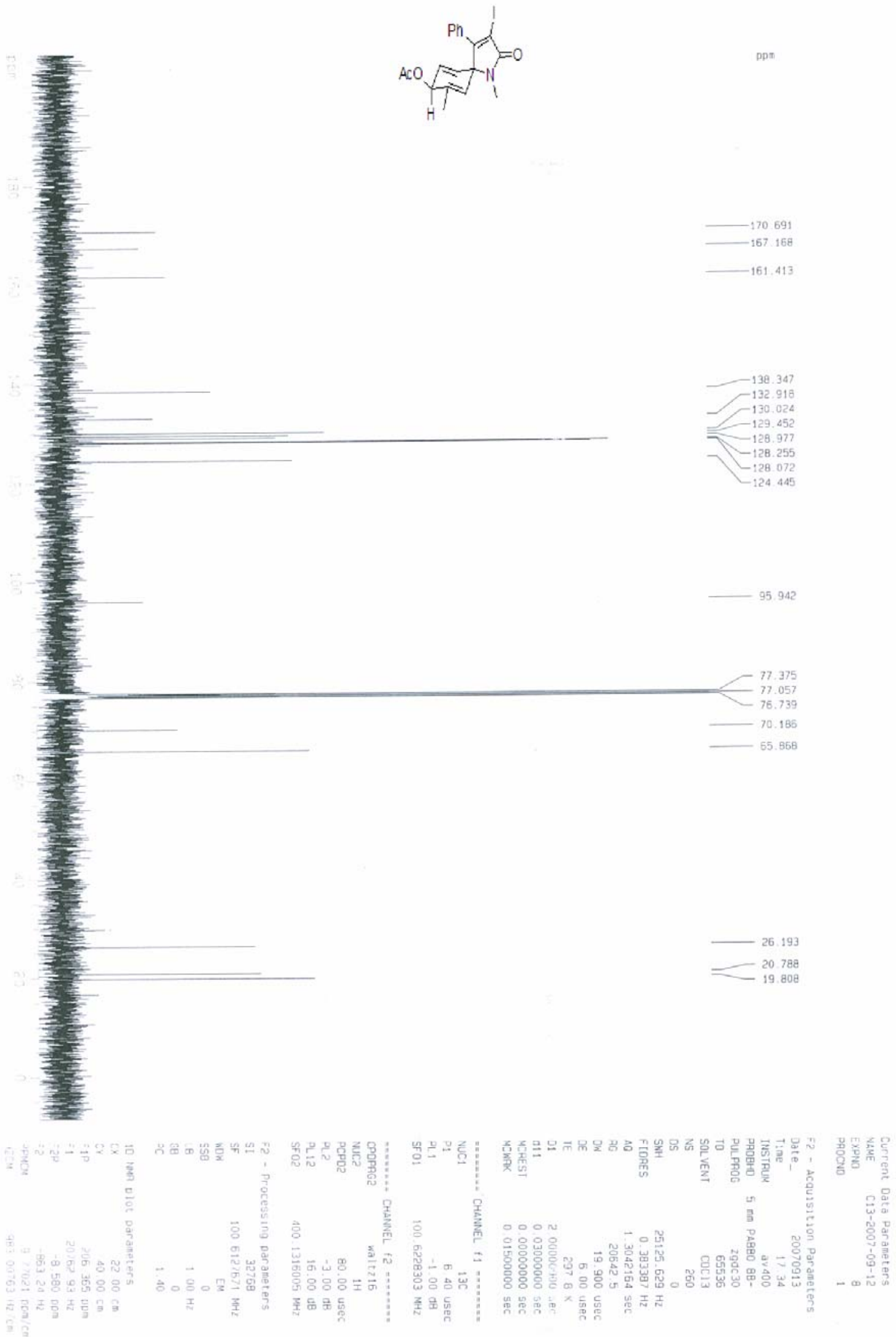
cis-N-Methyl-3-iodo-4-phenyl-6,9-dimethyl-1-azaspiro[4,5]-deca-3,6,9-trienyl 8-acetate (2g)

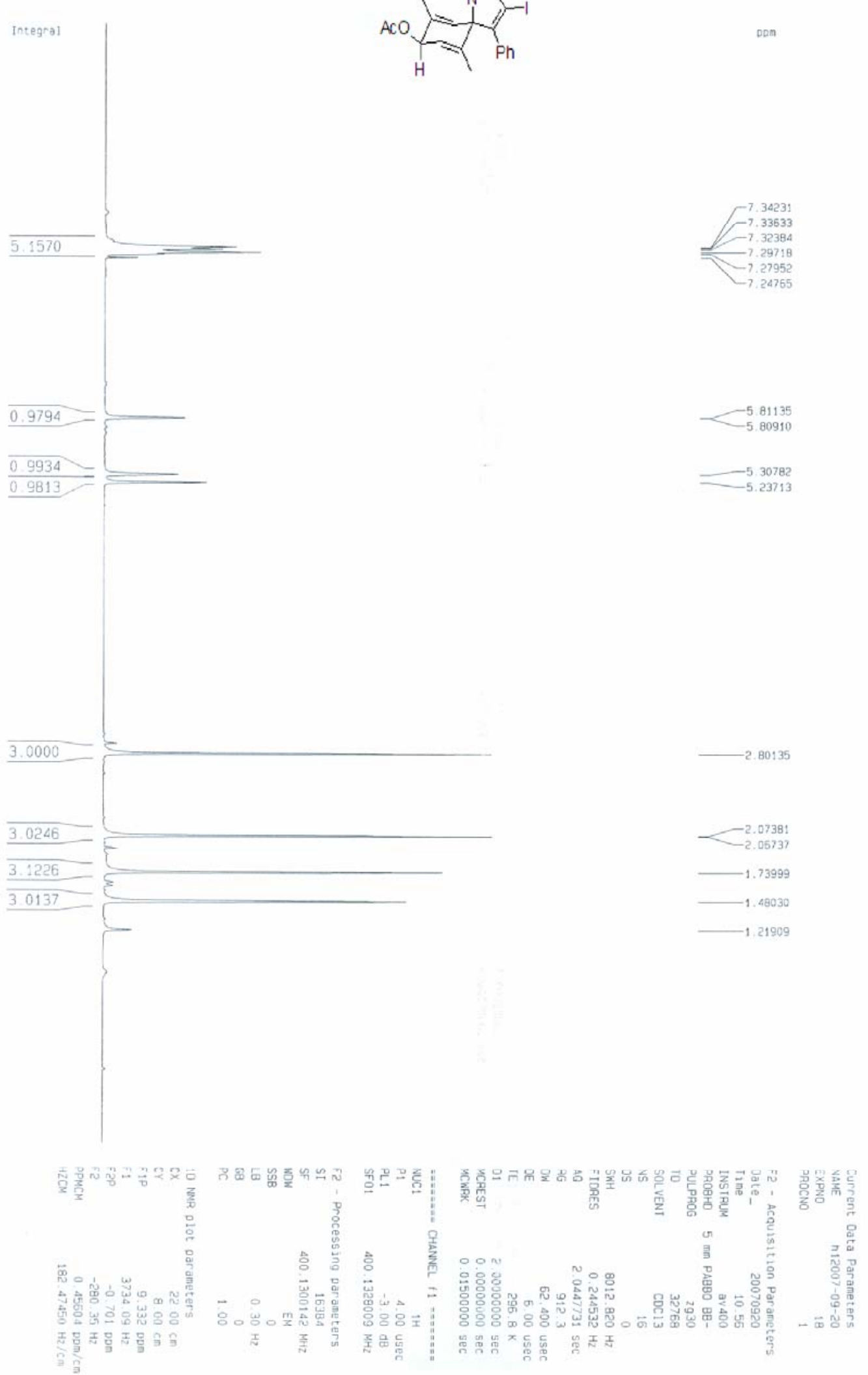


cis-N-Methyl-3-iodo-4-phenyl-6,9-dimethyl-1-azaspiro[4,5]-deca-3,6,9-trienyl 8-acetate (2g)
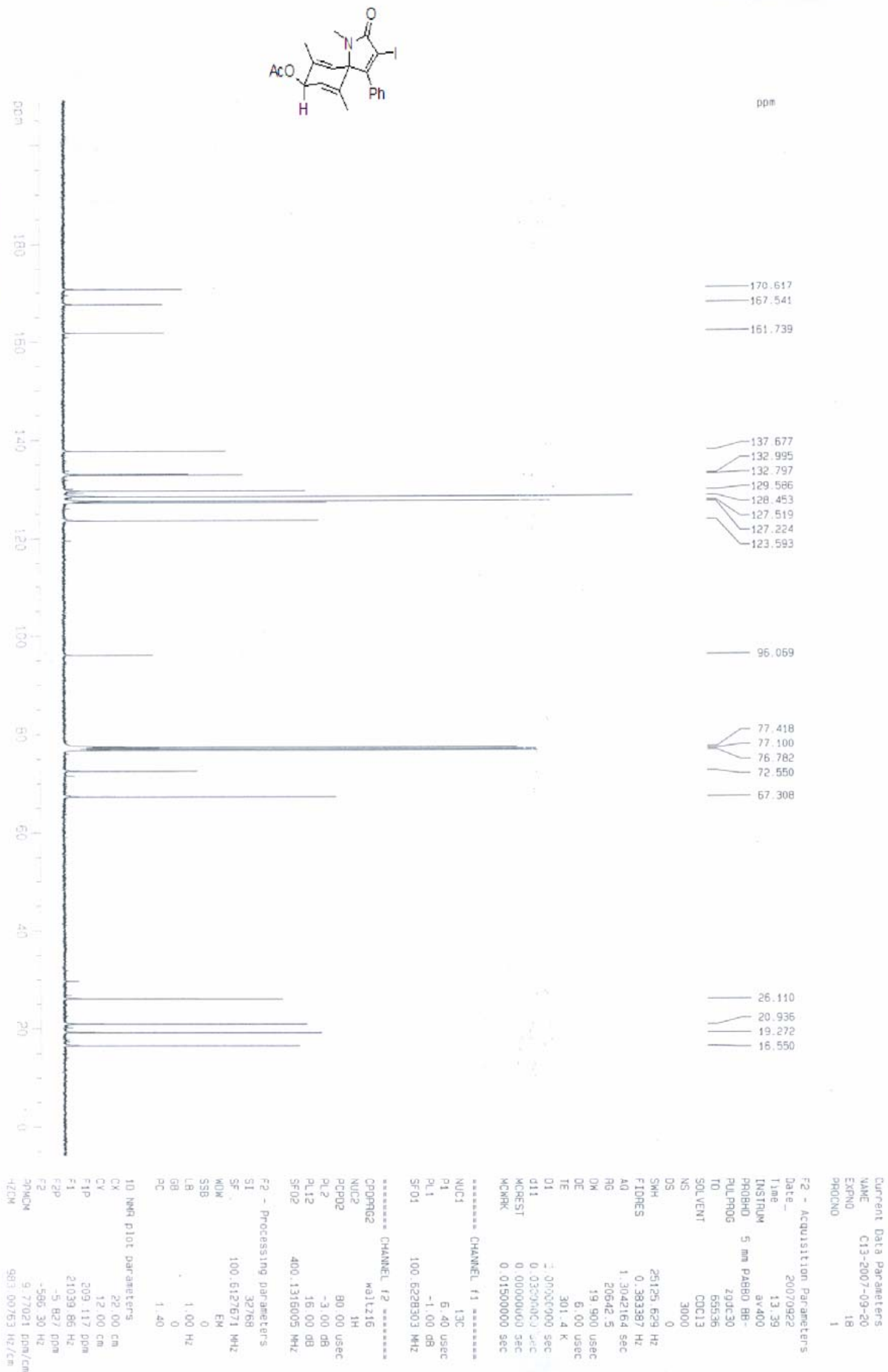
trans-N-Methyl-3-iodo-4-phenyl-6,9-dimethyl-1-azaspiro[4,5]-deca-3,6,9-trienyl 8-acetate (2g)
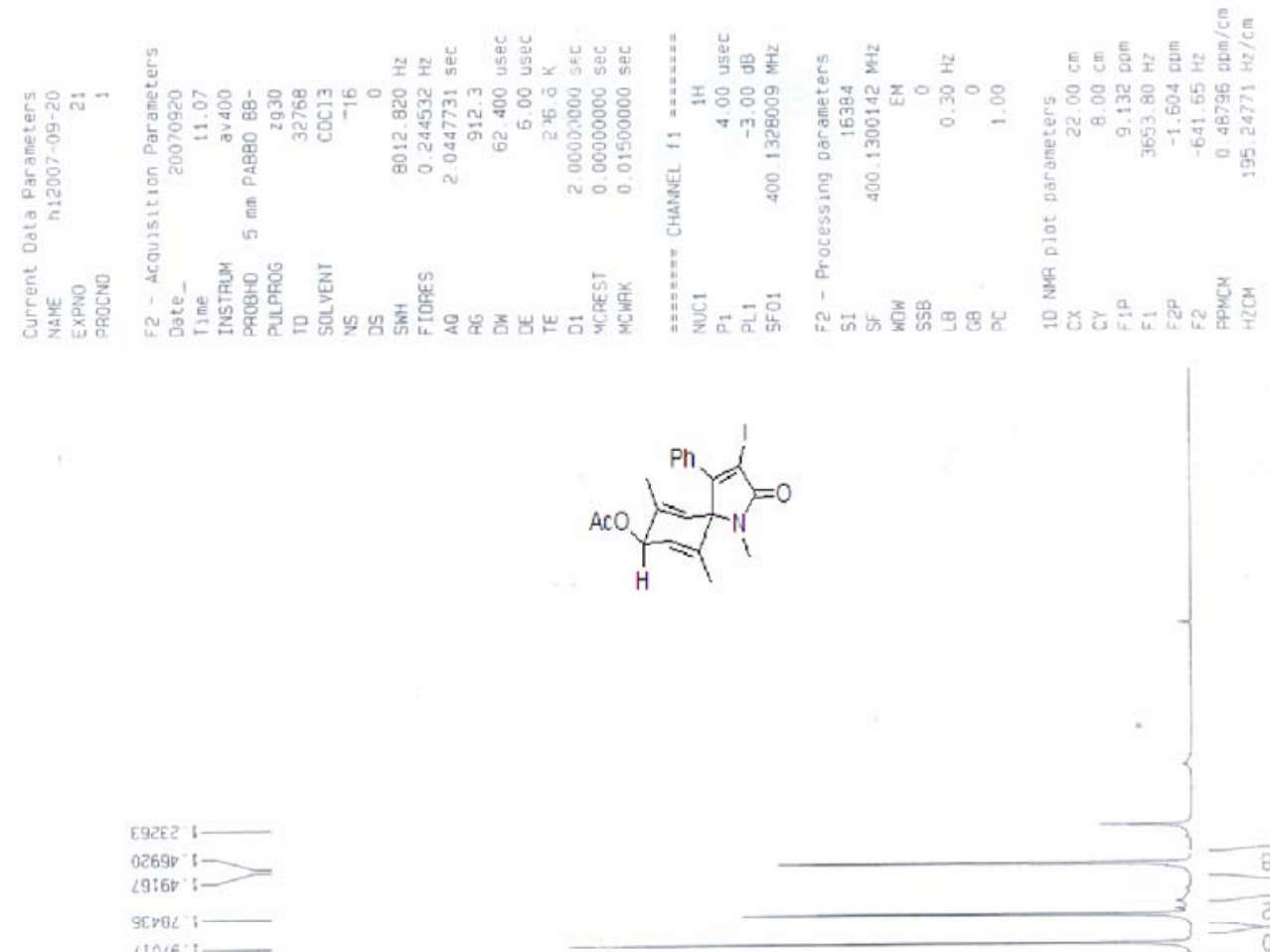

$410<6^{\circ}:$

$\varepsilon \angle O N L C$
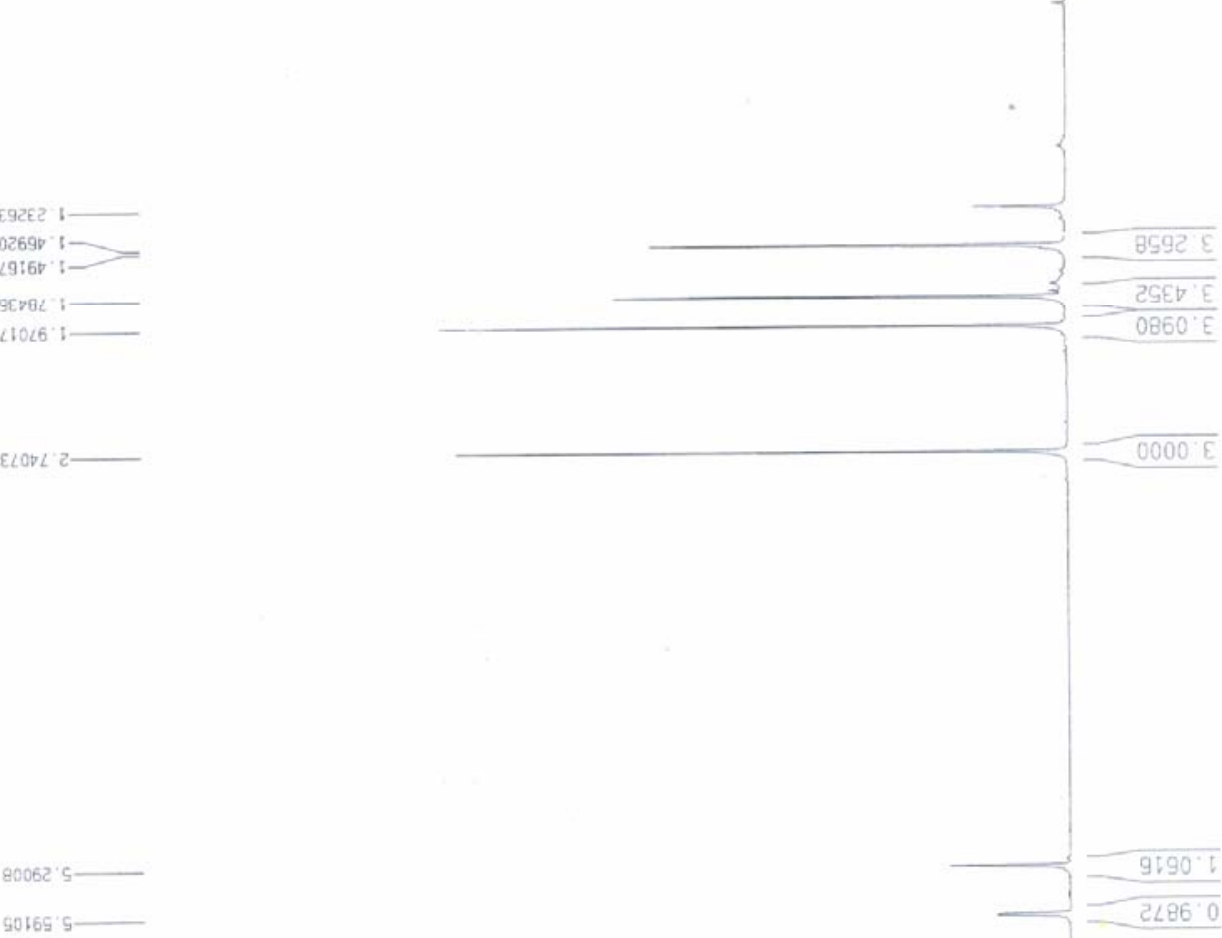

DBE $16: 5-2$

250265

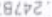

เsgeह $L-$

3909E: $2-1$

$99 \angle L E<$

Be65t $2-1$

(5) 4

दç $\angle 4<-$ 
trans-N-Methyl-3-iodo-4-phenyl-6,9-dimethyl-1-azaspiro[4,5]-deca-3,6,9-trienyl 8-acetate (2g)
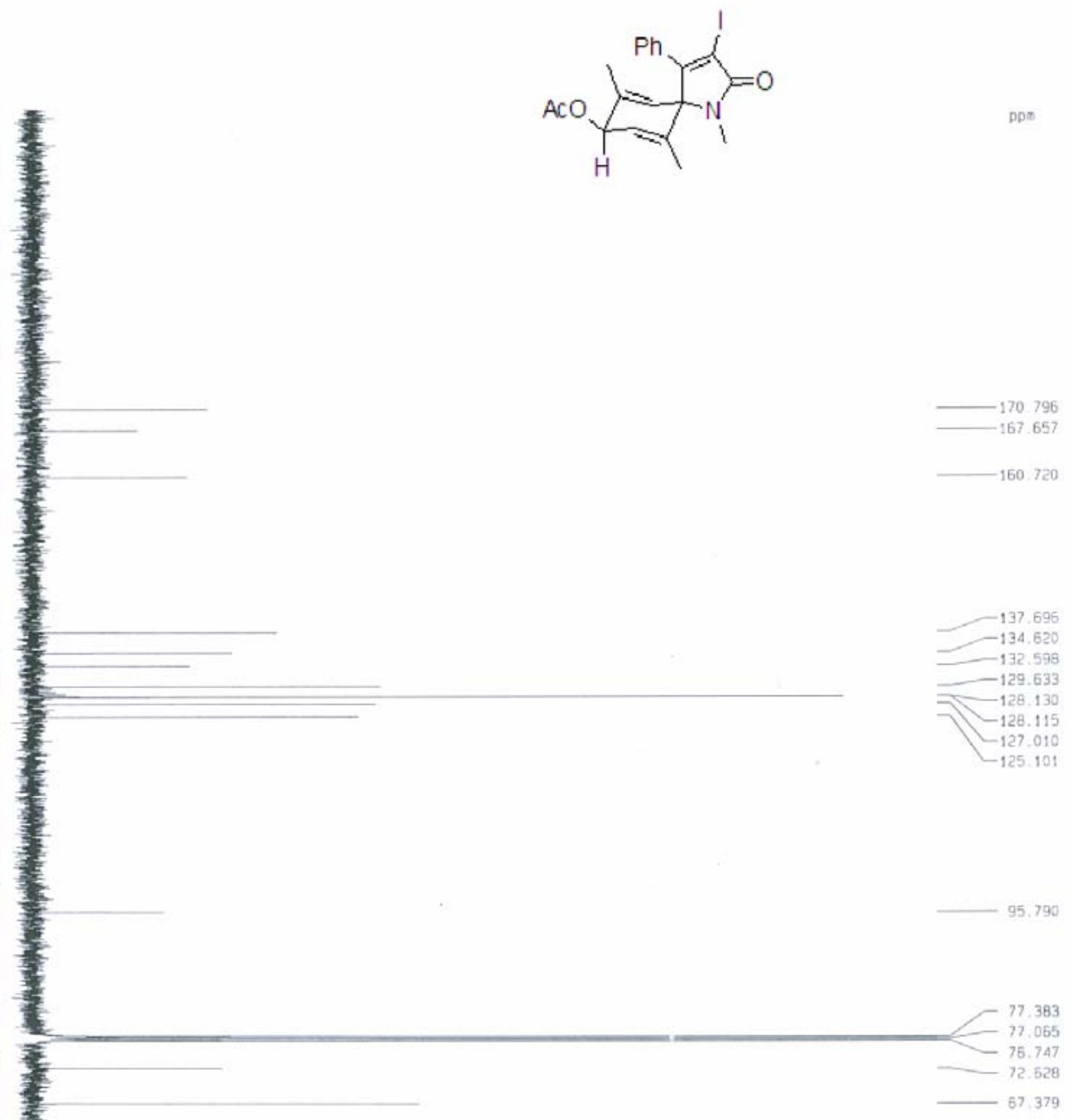

章新
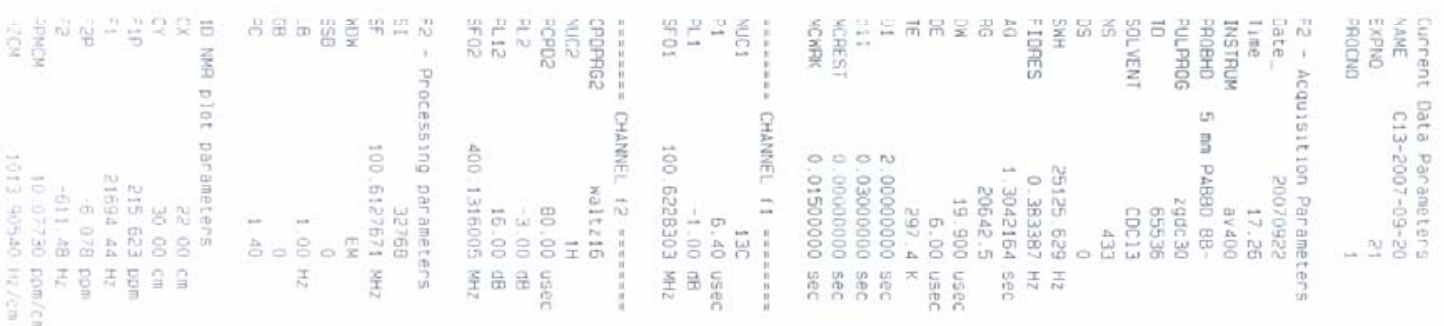
cis-N-Methyl-3-iodo-4-phenyl-6,7-dimethyl-1-azaspiro[4,5]-deca-3,6,9-trienyl 8-acetate (2h)

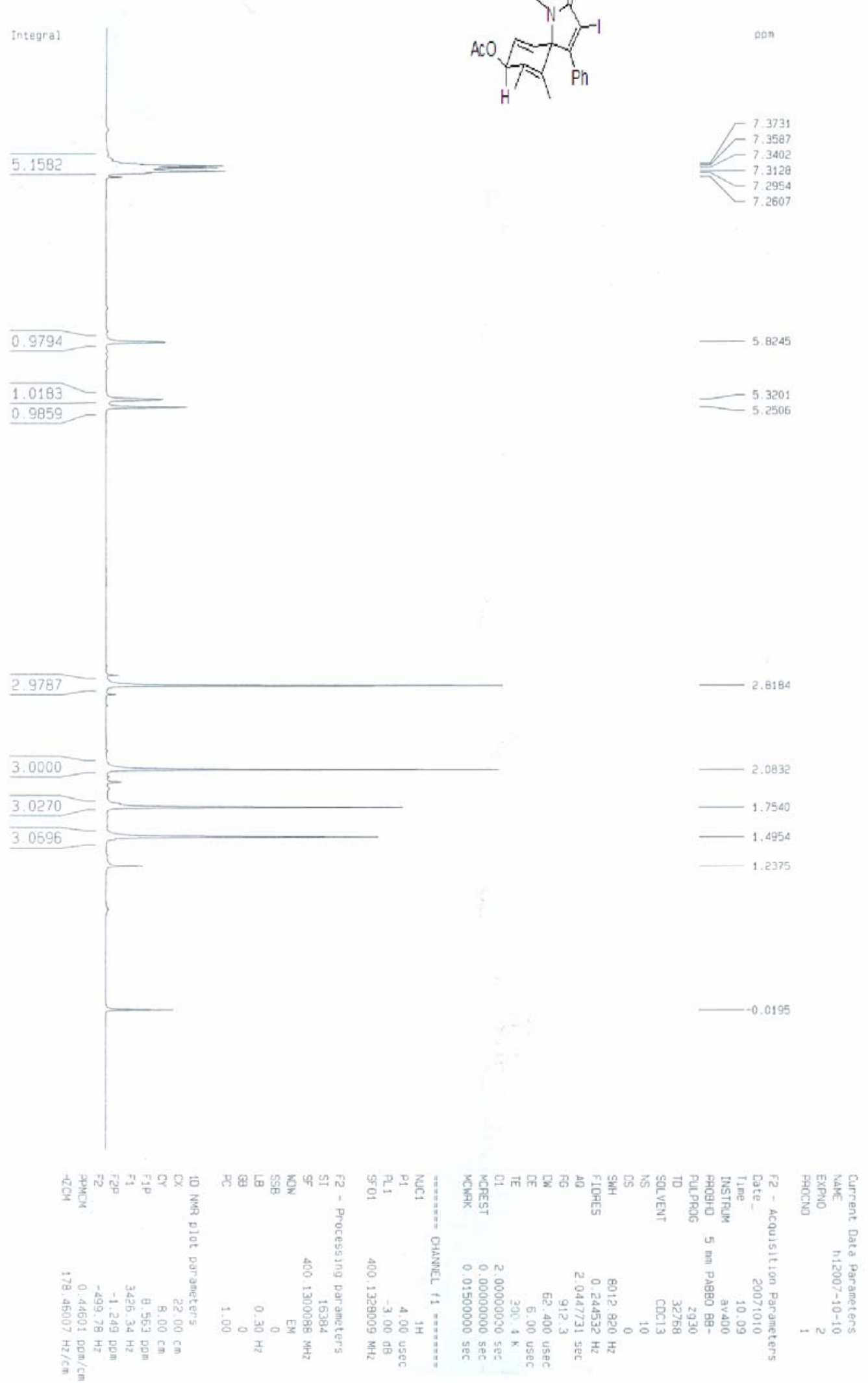



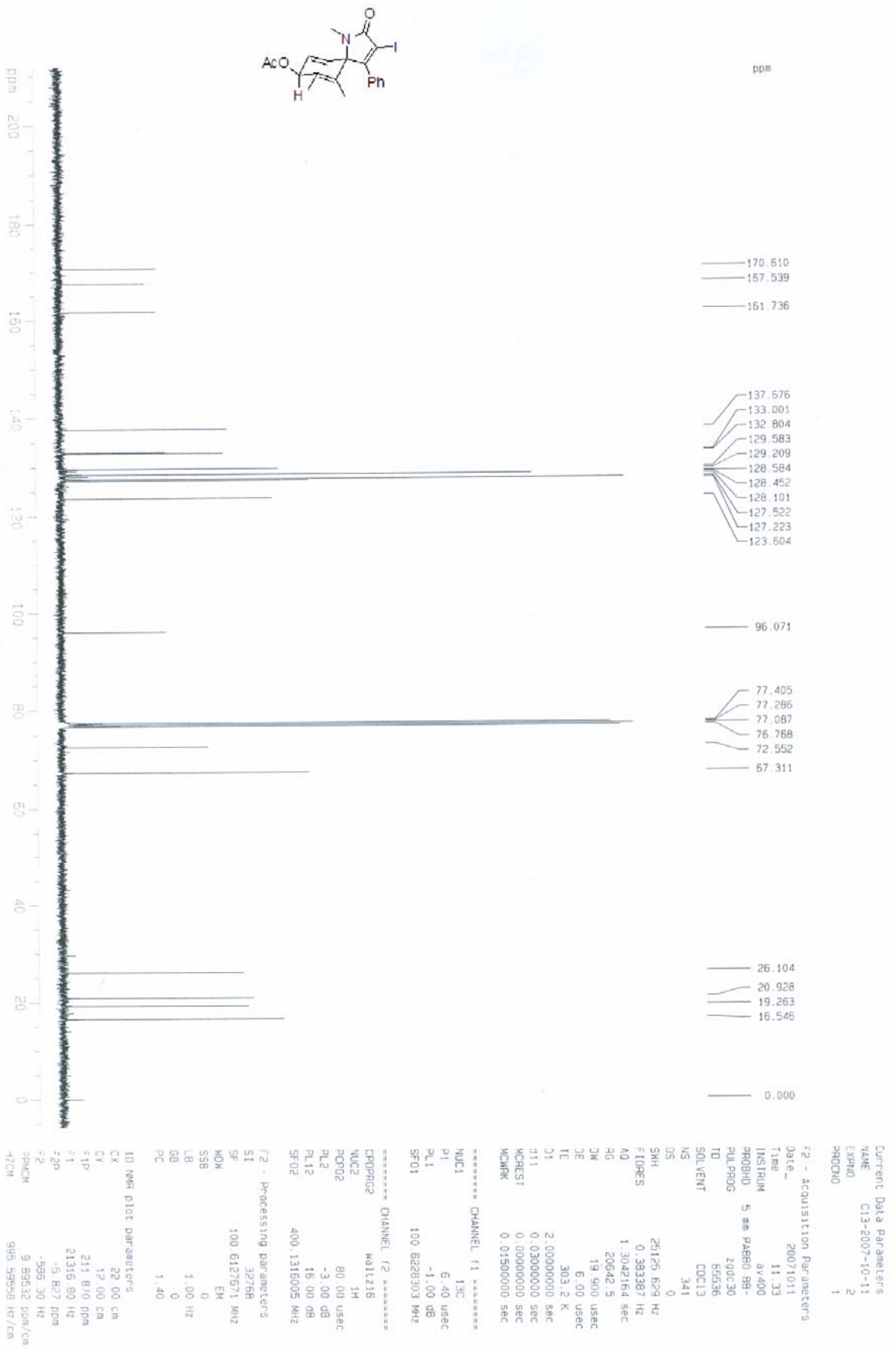
trans-N-Methyl-3-iodo-4-phenyl-6,7-dimethyl-1-azaspiro[4,5]-deca-3,6,9-trienyl 8-acetate (2h)

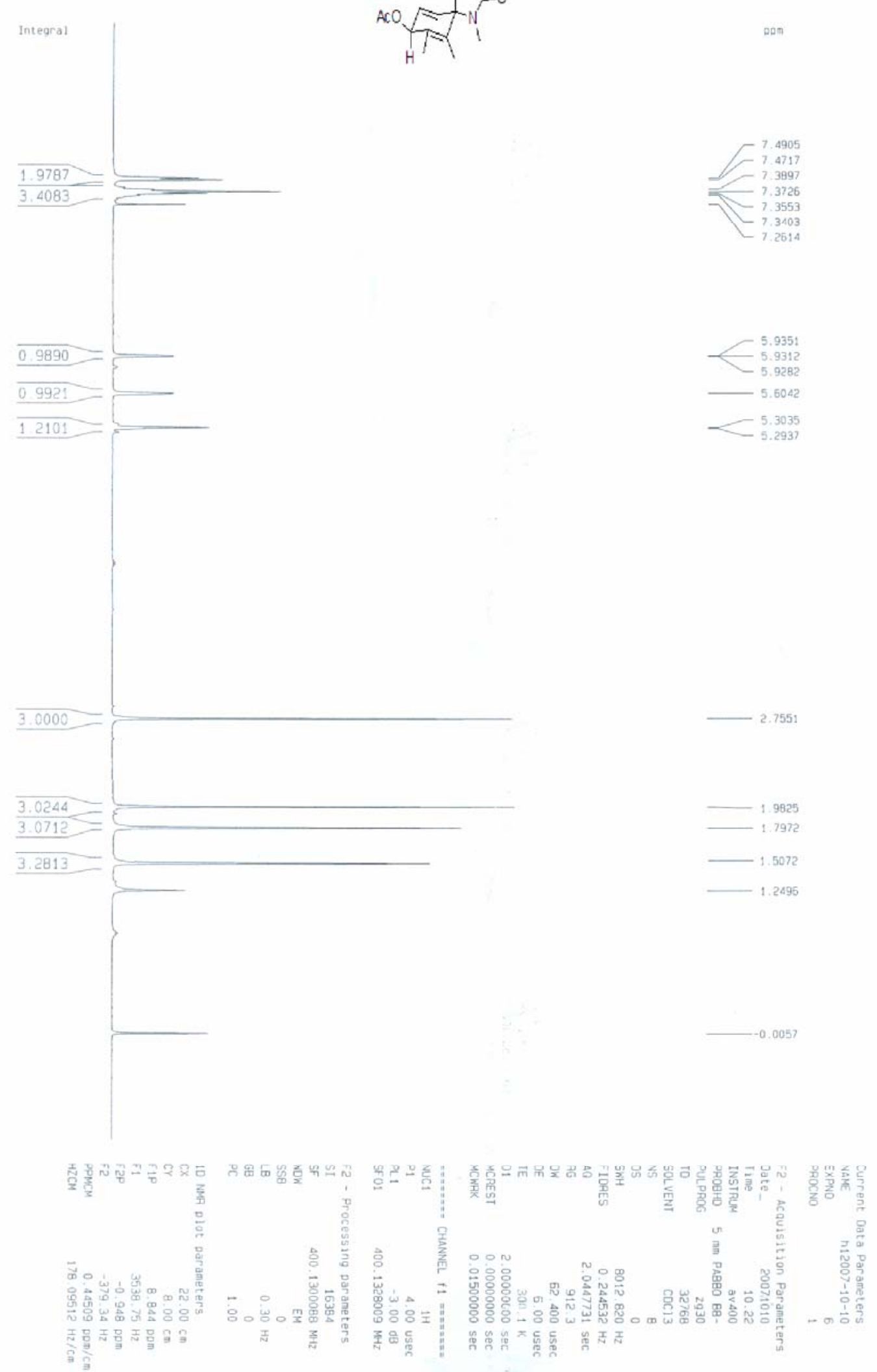


trans-N-Methyl-3-iodo-4-phenyl-6,7-dimethyl-1-azaspiro[4,5]-deca-3,6,9-trienyl 8-acetate (2h)

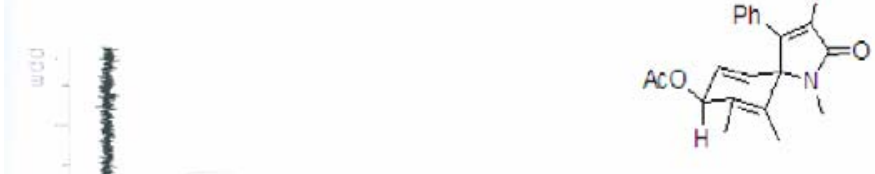

ppm

-170.722
-167.617

$-150,718$

$5-\frac{\frac{1}{4}}{\frac{1}{3}}$

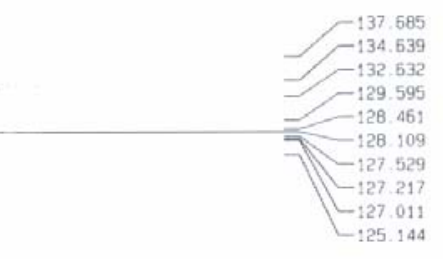

95.804

8

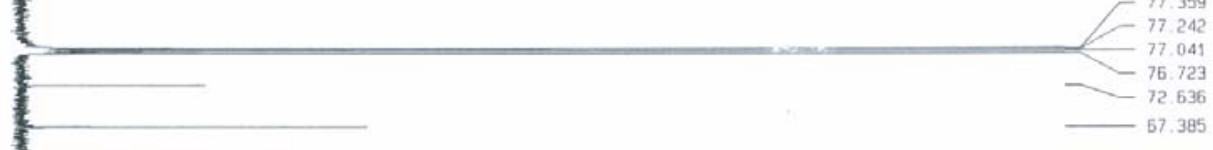

8
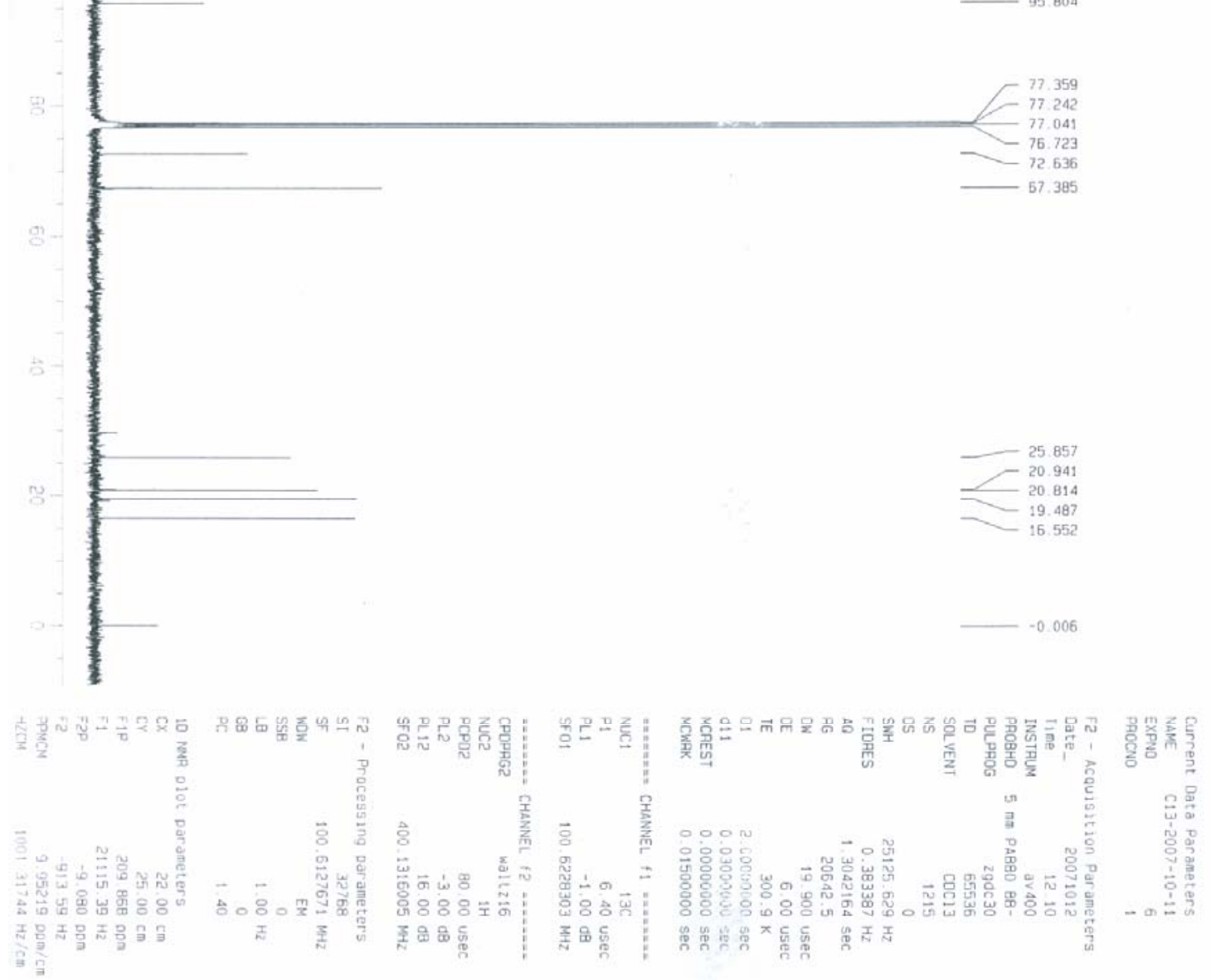

S29 
cis-N-Methyl-3-iodo-4-phenyl-6-chloro-1-azaspiro[4,5]-deca-3,6,9-trienyl 8-acetate (2i)

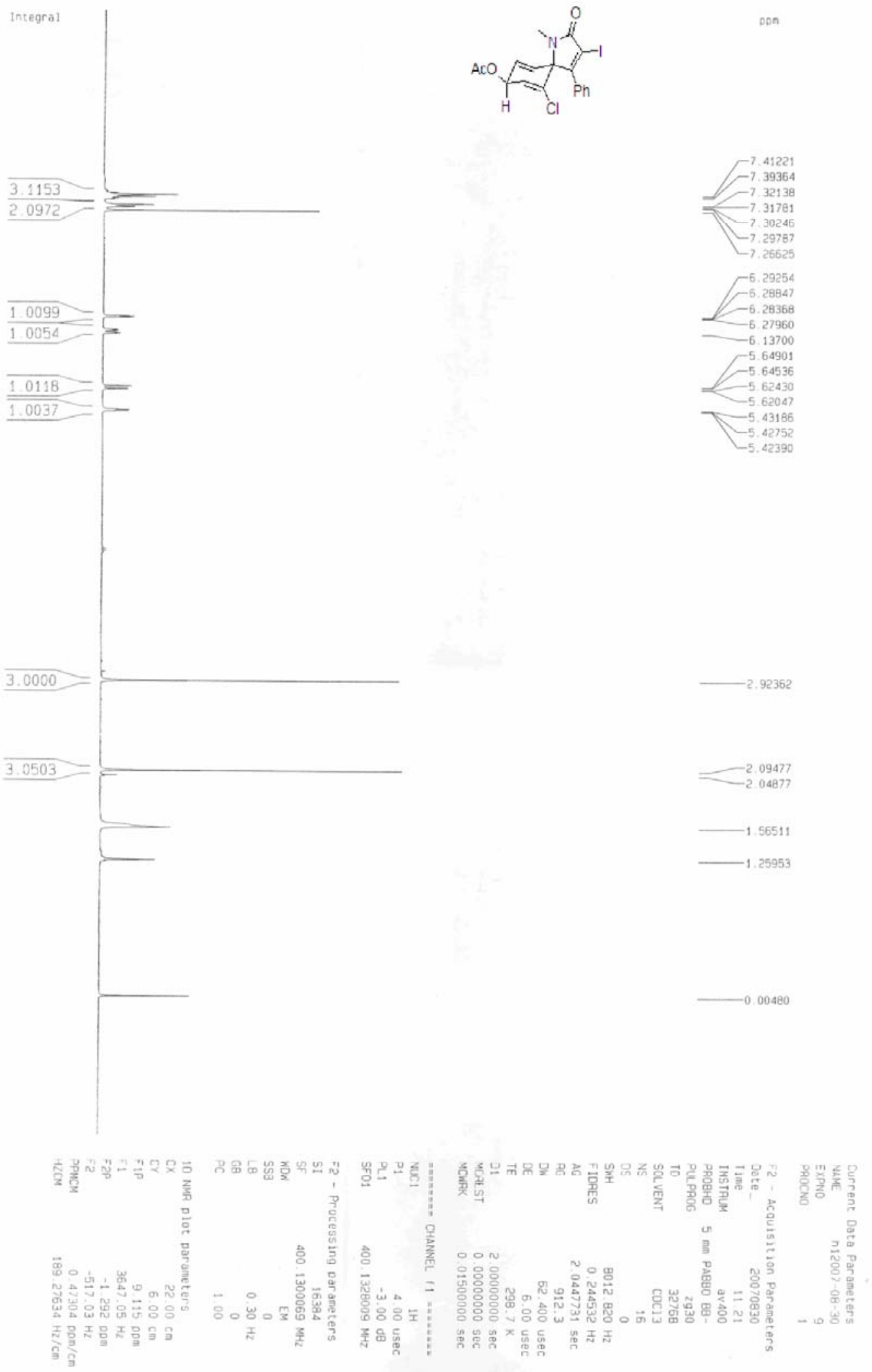


cis-N-Methyl-3-iodo-4-phenyl-6-chloro-1-azaspiro[4,5]-deca-3,6,9-trienyl 8-acetate (2i)

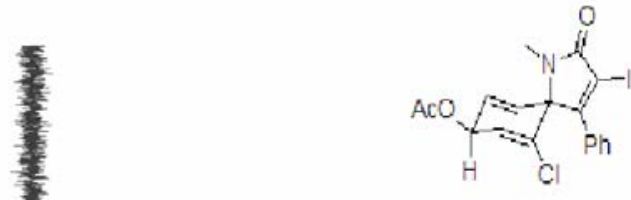

170.169
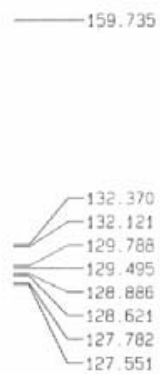

98.167
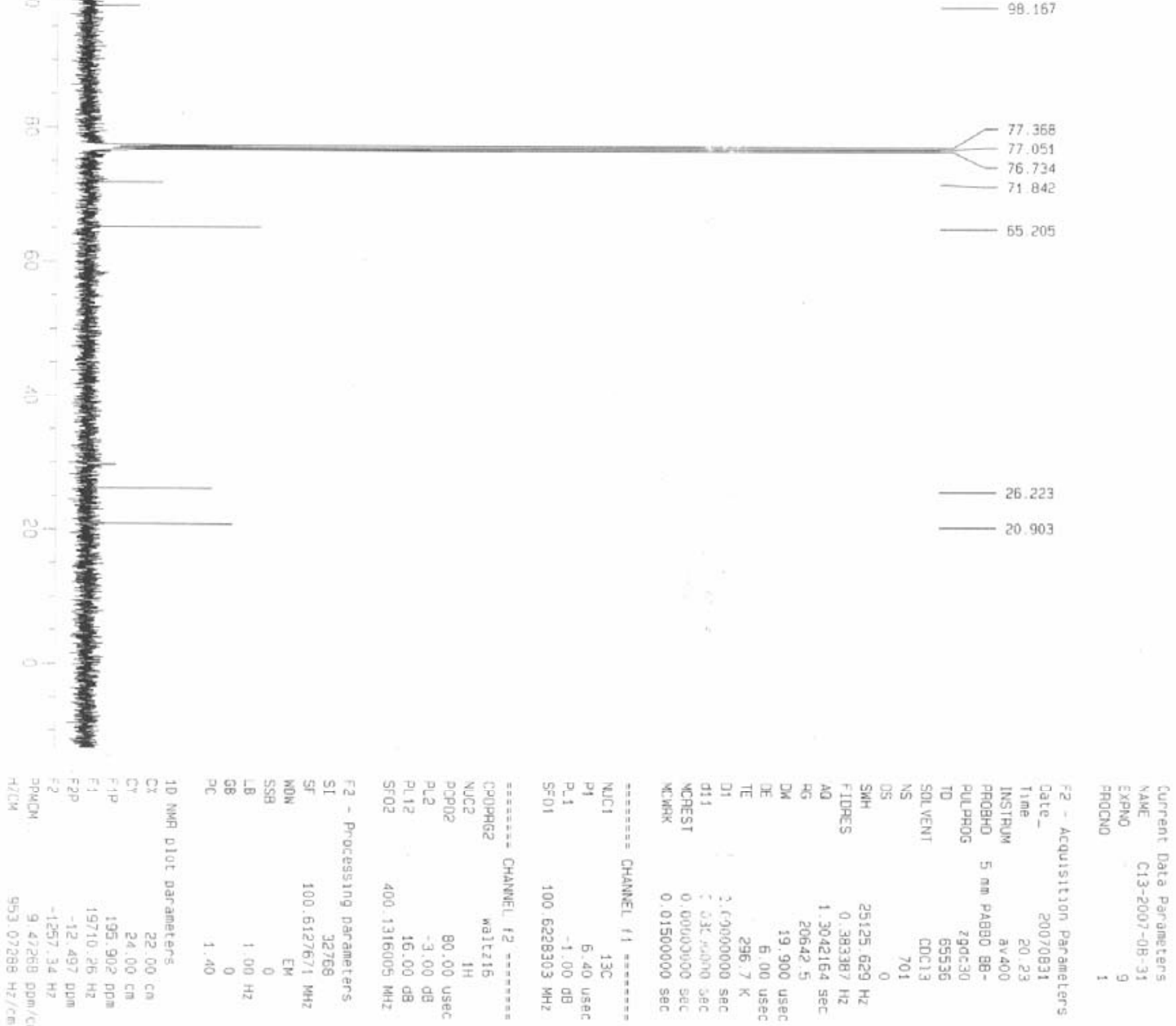
trans-N-Methyl-3-iodo-4-phenyl-6-chloro-1-azaspiro[4,5]-deca-3,6,9-trienyl 8-acetate (2i)

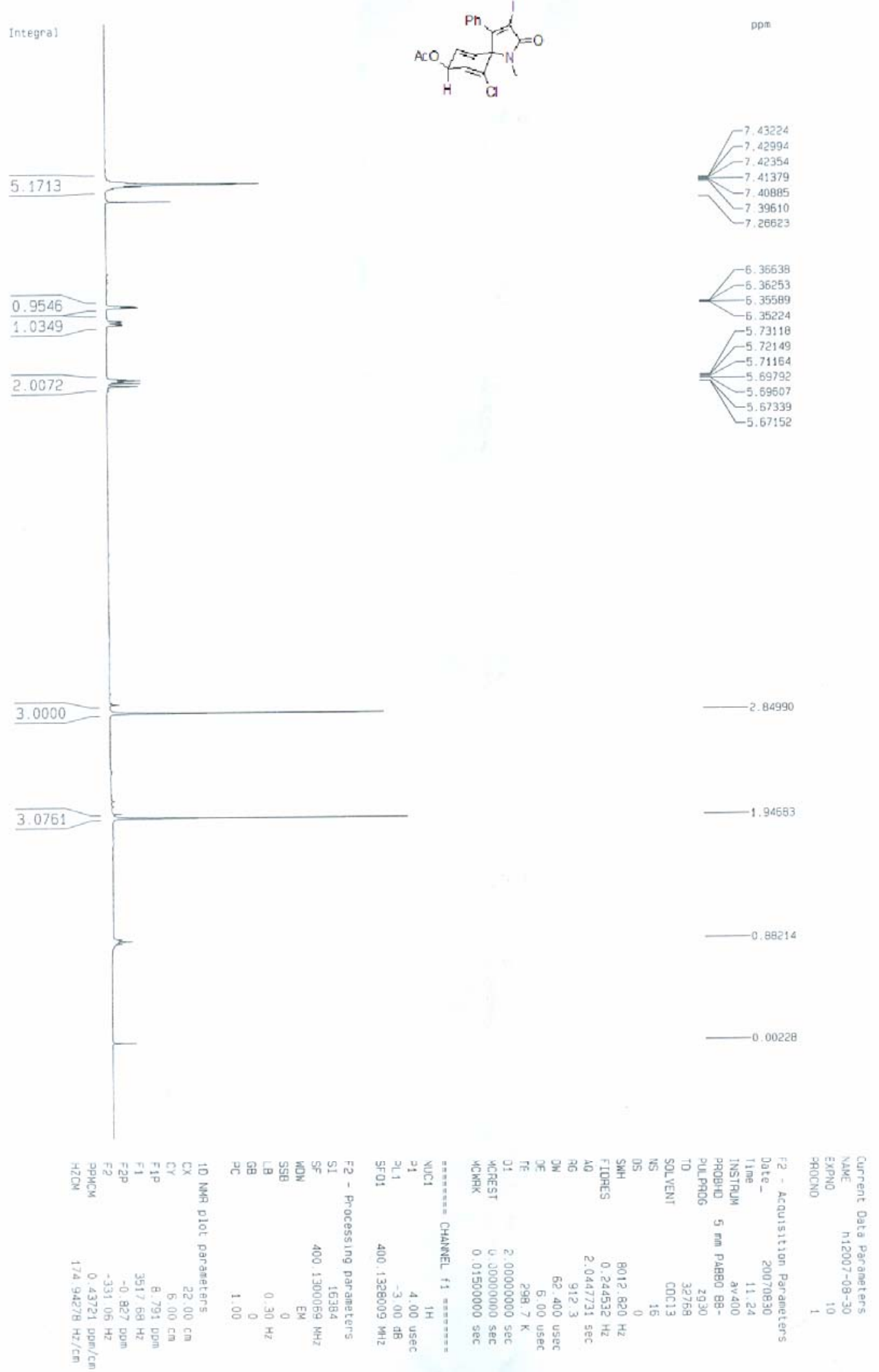


trans-N-Methyl-3-iodo-4-phenyl-6-chloro-1-azaspiro[4,5]-deca-3,6,9-trienyl 8-acetate (2i)
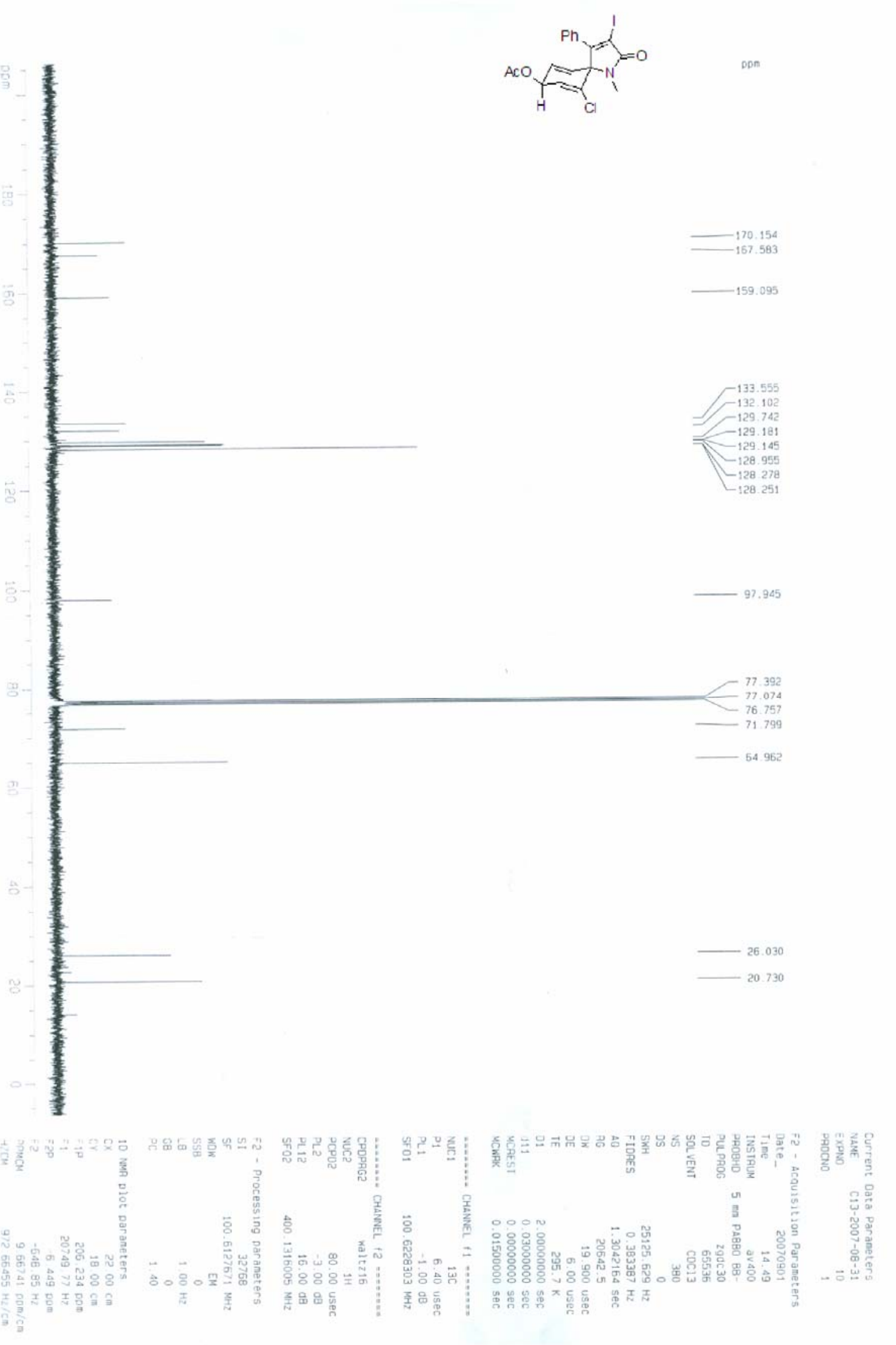
cis-N-Methyl-3-iodo-4-phenyl-6-bromo-1-azaspiro[4,5]-deca-3,6,9-trienyl 8-acetate (2j)

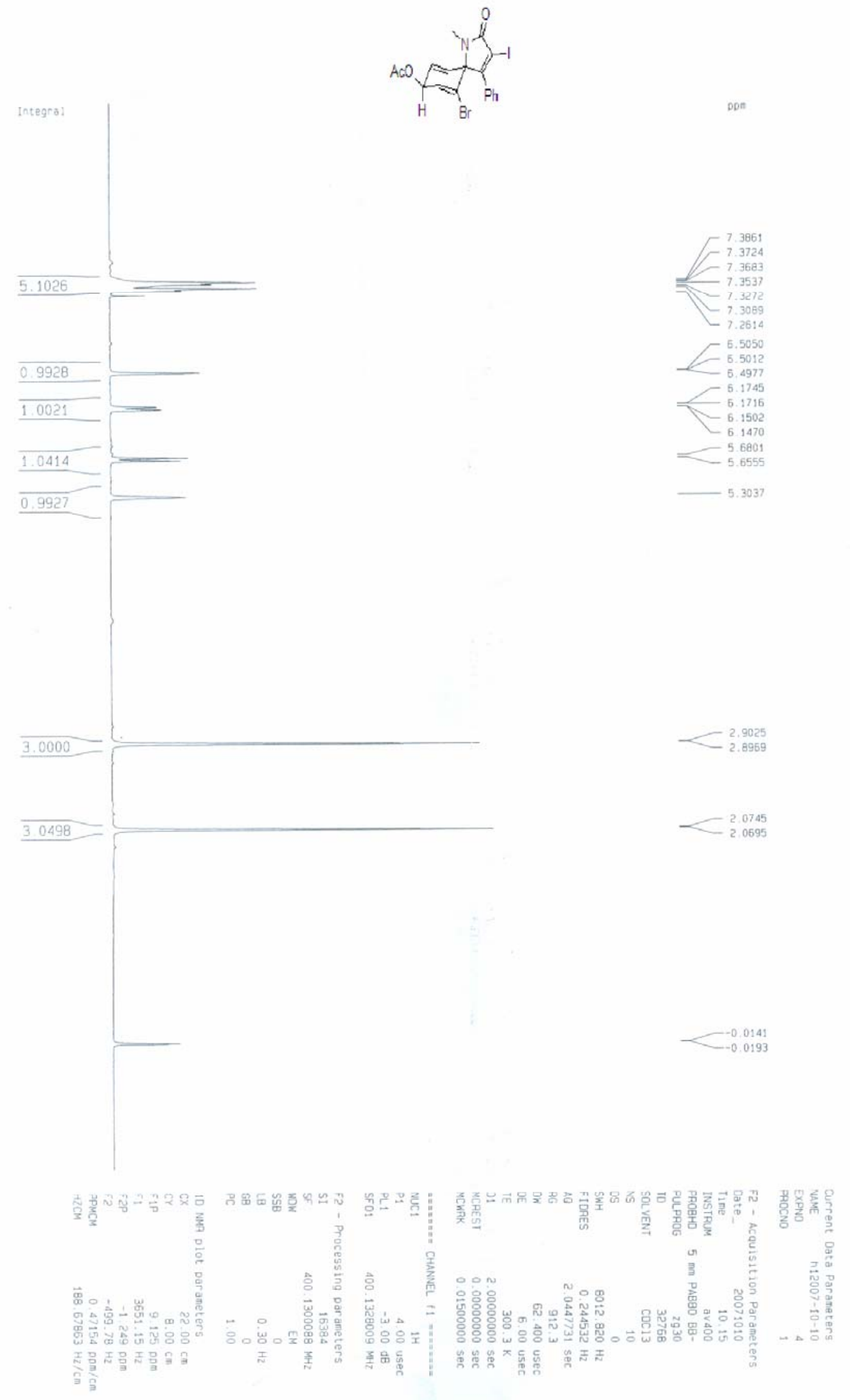


cis-N-Methyl-3-iodo-4-phenyl-6-bromo-1-azaspiro[4,5]-deca-3,6,9-trienyl 8-acetate (2j)
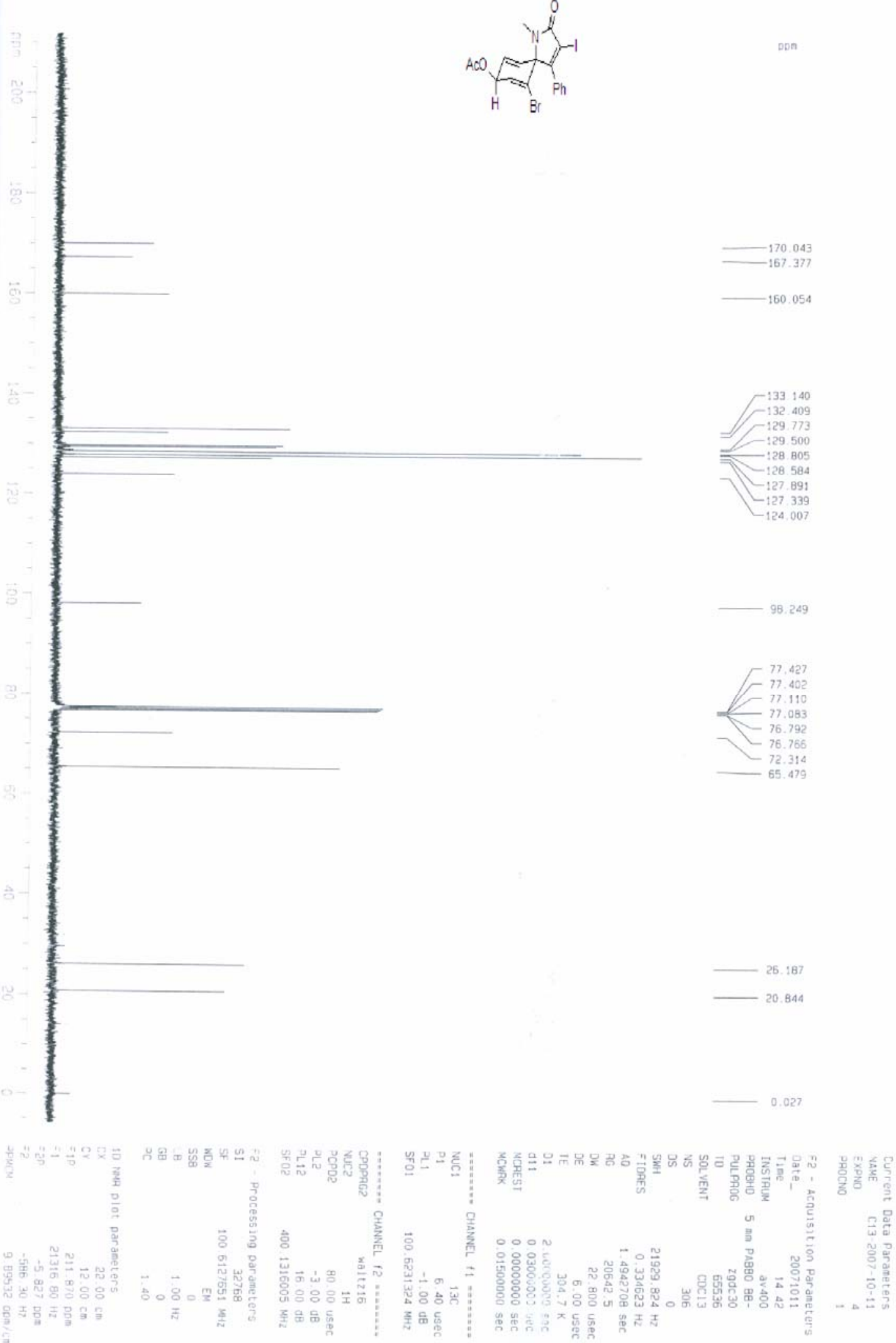
trans-N-Methyl-3-iodo-4-phenyl-6-bromo-1-azaspiro[4,5]-deca-3,6,9-trienyl 8-acetate (2j)

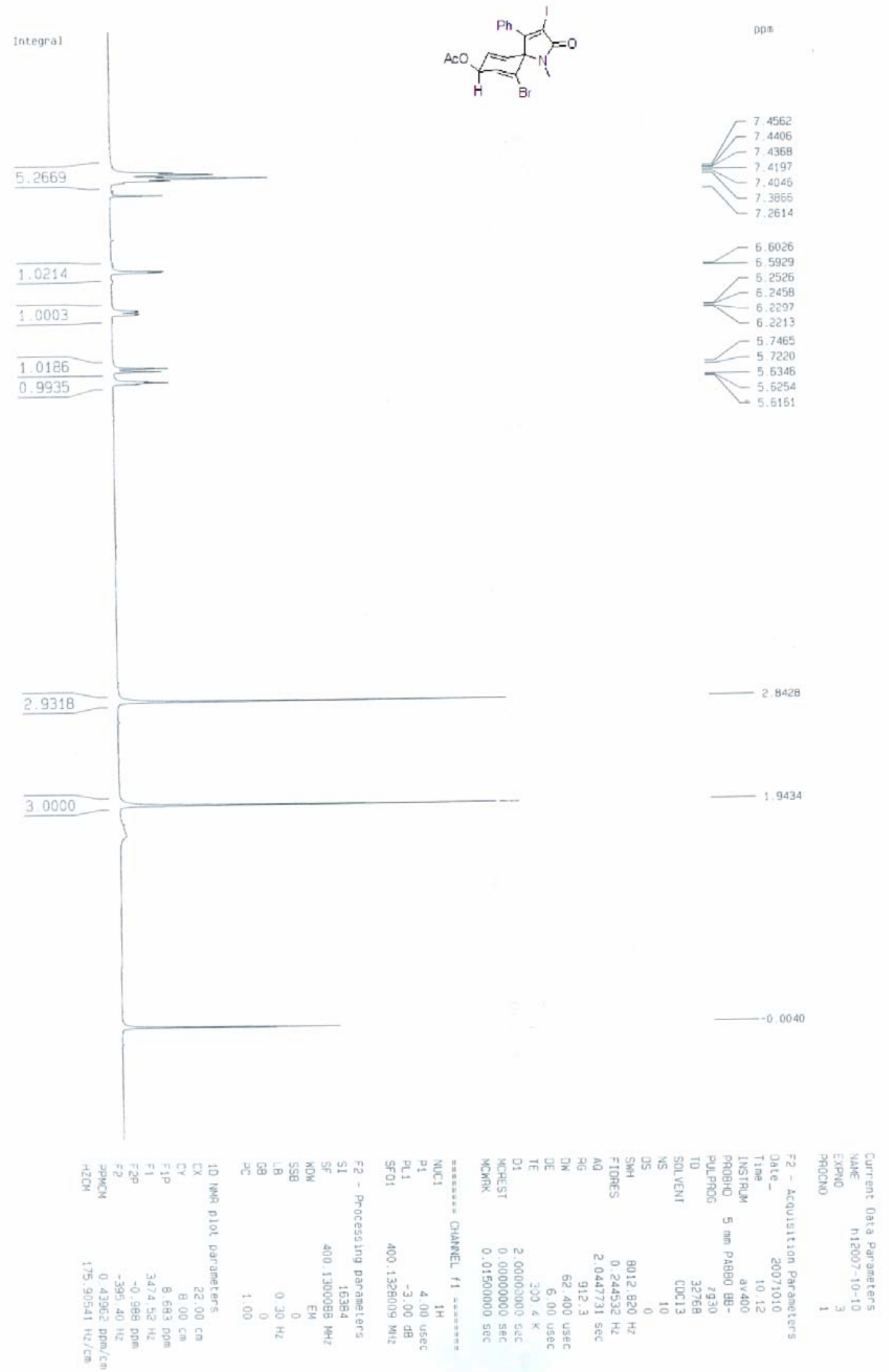


trans-N-Methyl-3-iodo-4-phenyl-6-bromo-1-azaspiro[4,5]-deca-3,6,9-trienyl 8-acetate (2j)
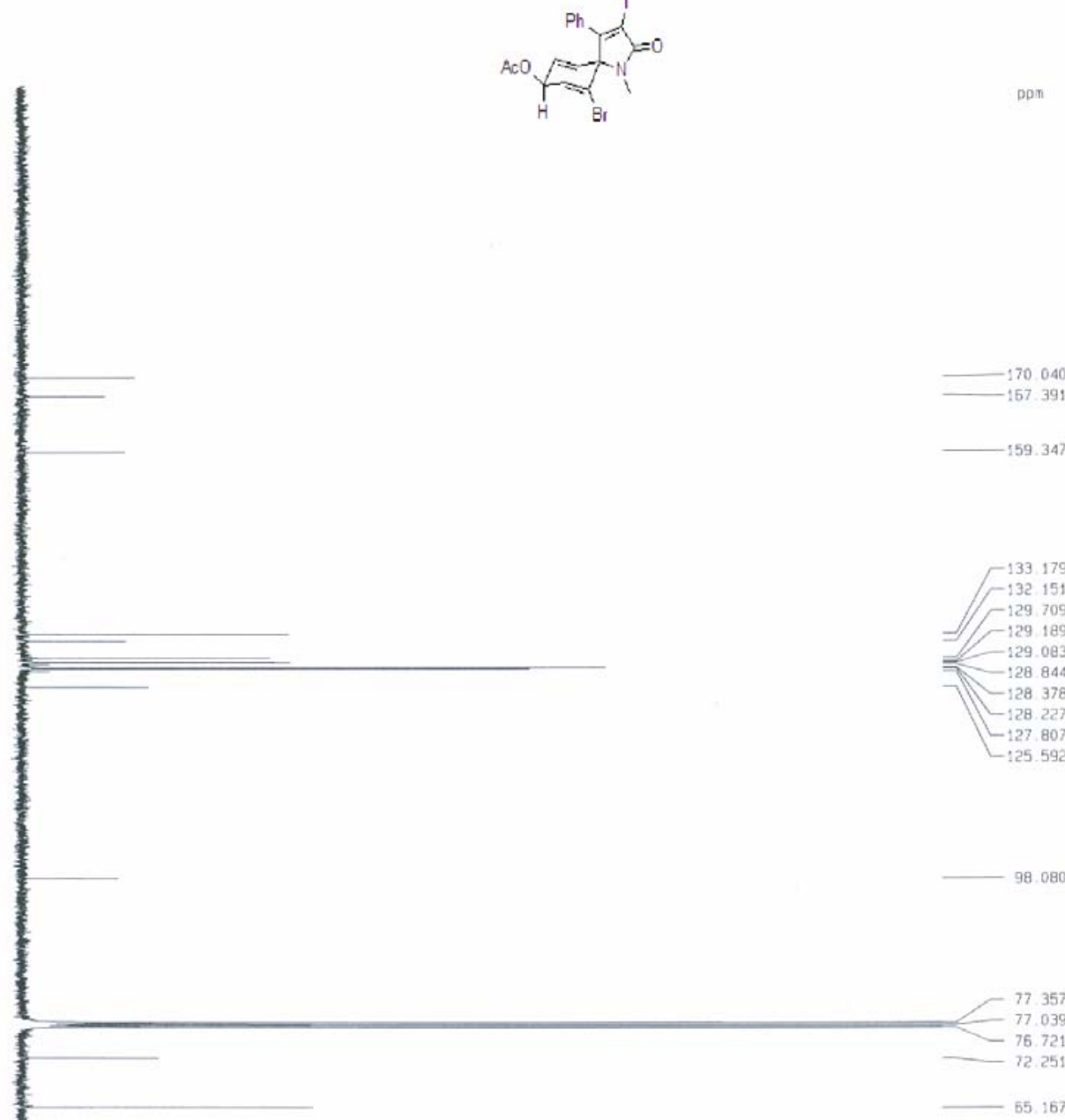
cis-N-Methyl-3,6-diiodo-4-phenyl-1-azaspiro[4,5]-deca-3,6,9-trienyl 8-acetate (2k)

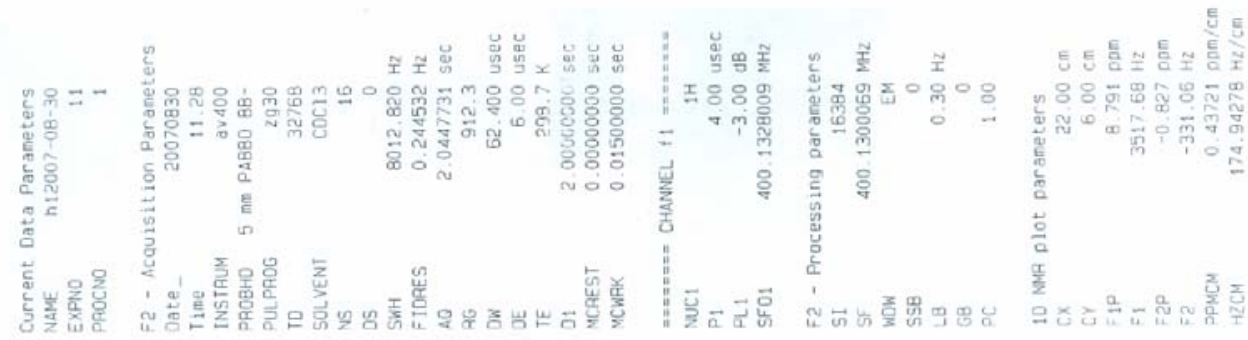

$8 E 100 \cdot 0-$
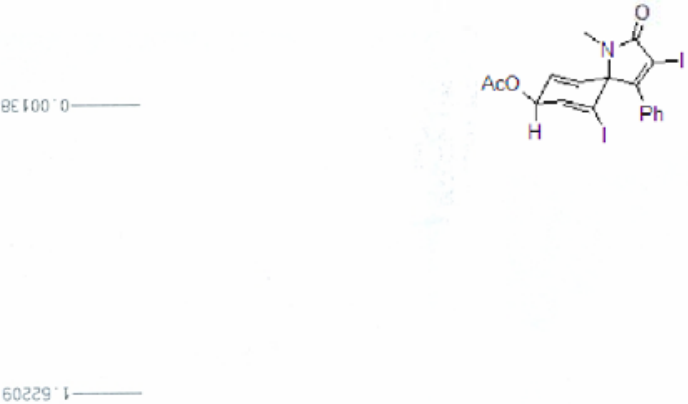

$10160^{\circ} 2$

$\angle 9216: 2-$

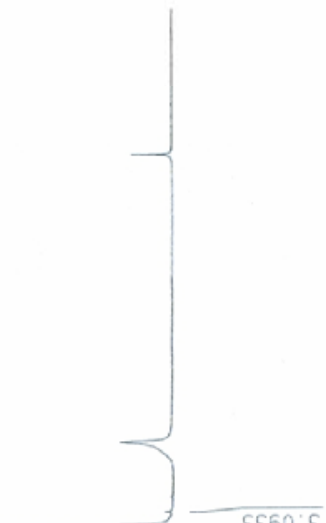

.

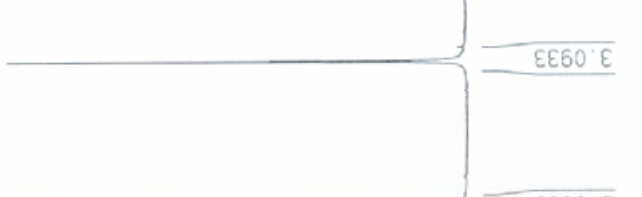

$029615-$

$\angle 10025$

$96025-9$

EGGL' S-

$\triangle D O O L C$

ट६ร9L' $\mathrm{S}$

$\varepsilon 0696$ '

$61<02.9-7$

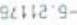

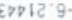

26015.9-

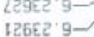

LDEV 9

$0096<$ ' 9

टह56 9

62E08 9

$29 \angle 00-9$

$9029 E^{\prime}:-$

द्वVE: :-

89608

256002

8LDto 2

udd

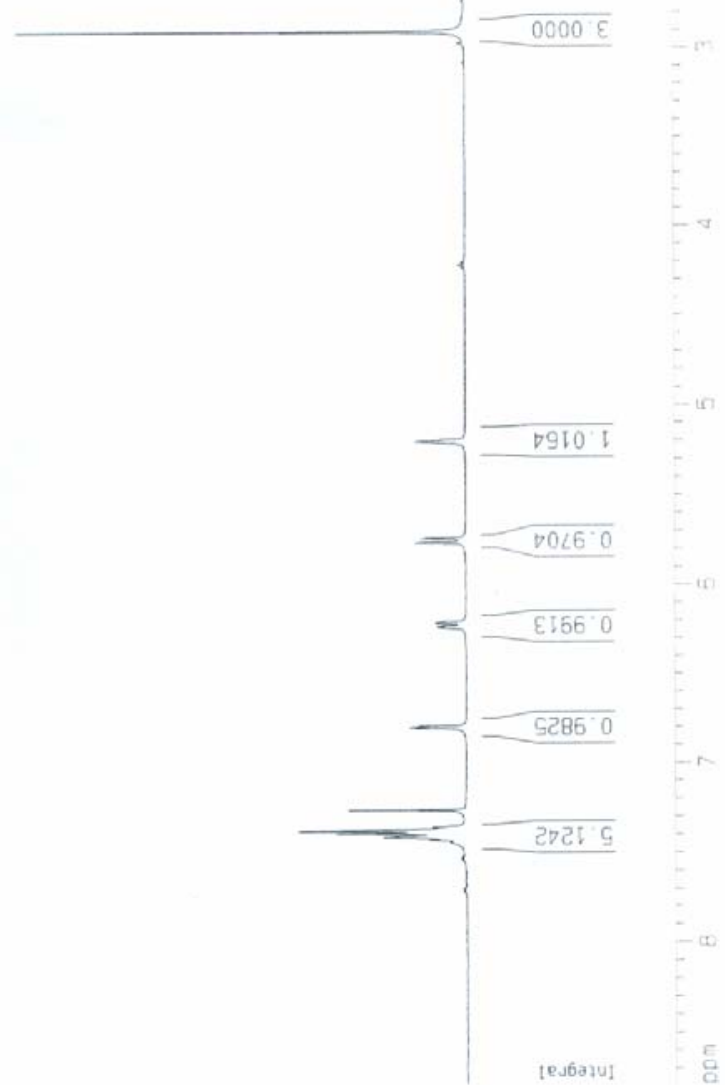


cis-N-methyl-3,6-diiodo-4-phenyl-1-azaspiro[4,5]-deca-3,6,9-trienyl 8-acetate (2k)

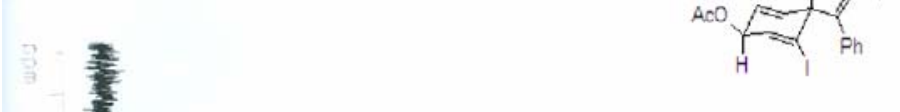

$$
\begin{array}{r}
-170.112 \\
-167.205 \\
-160.561
\end{array}
$$
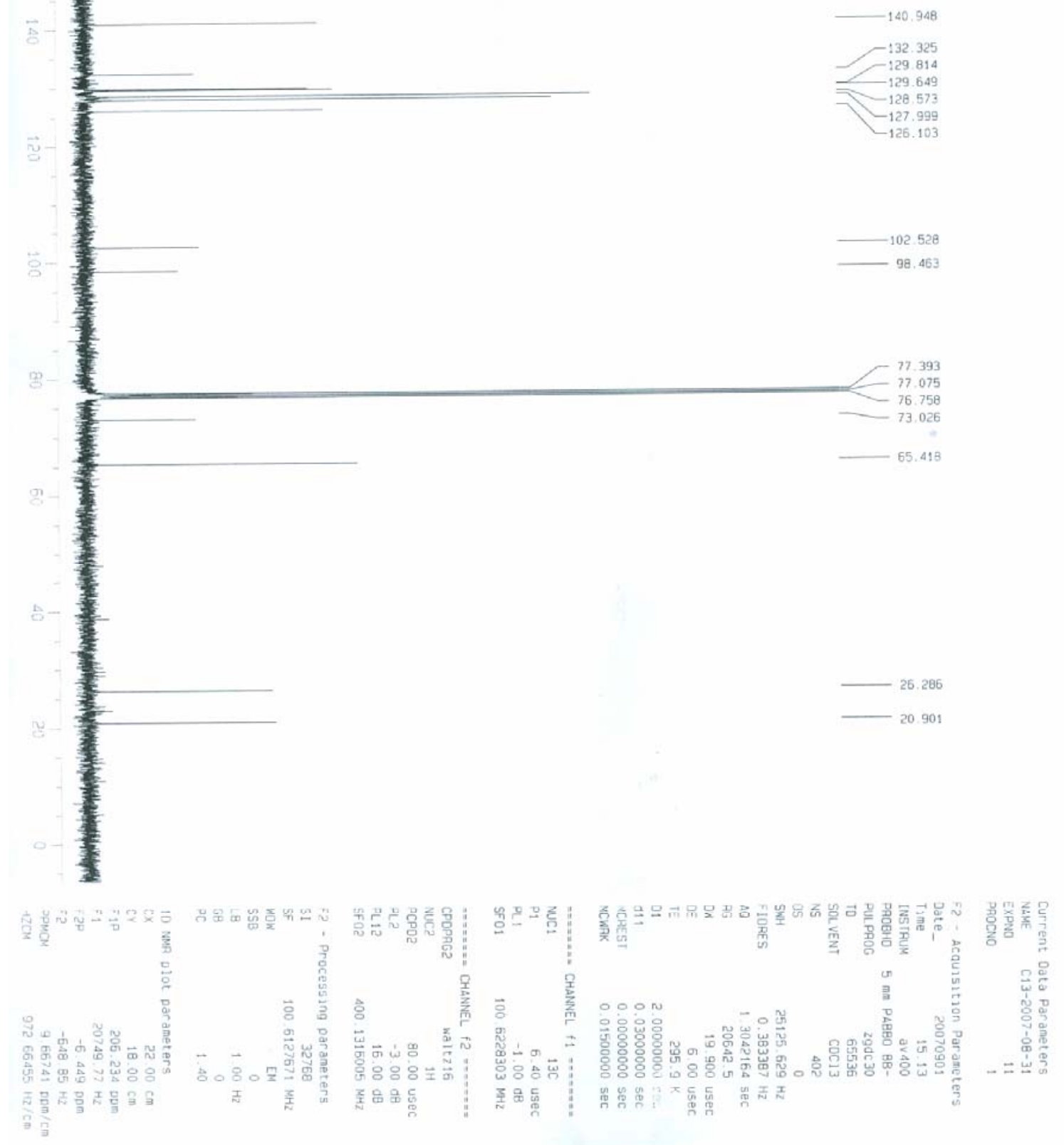

S39 
trans-N-methyl-3,6-diiodo-4-phenyl-1-azaspiro[4,5]-deca-3,6,9-trienyl 8-acetate (2k)

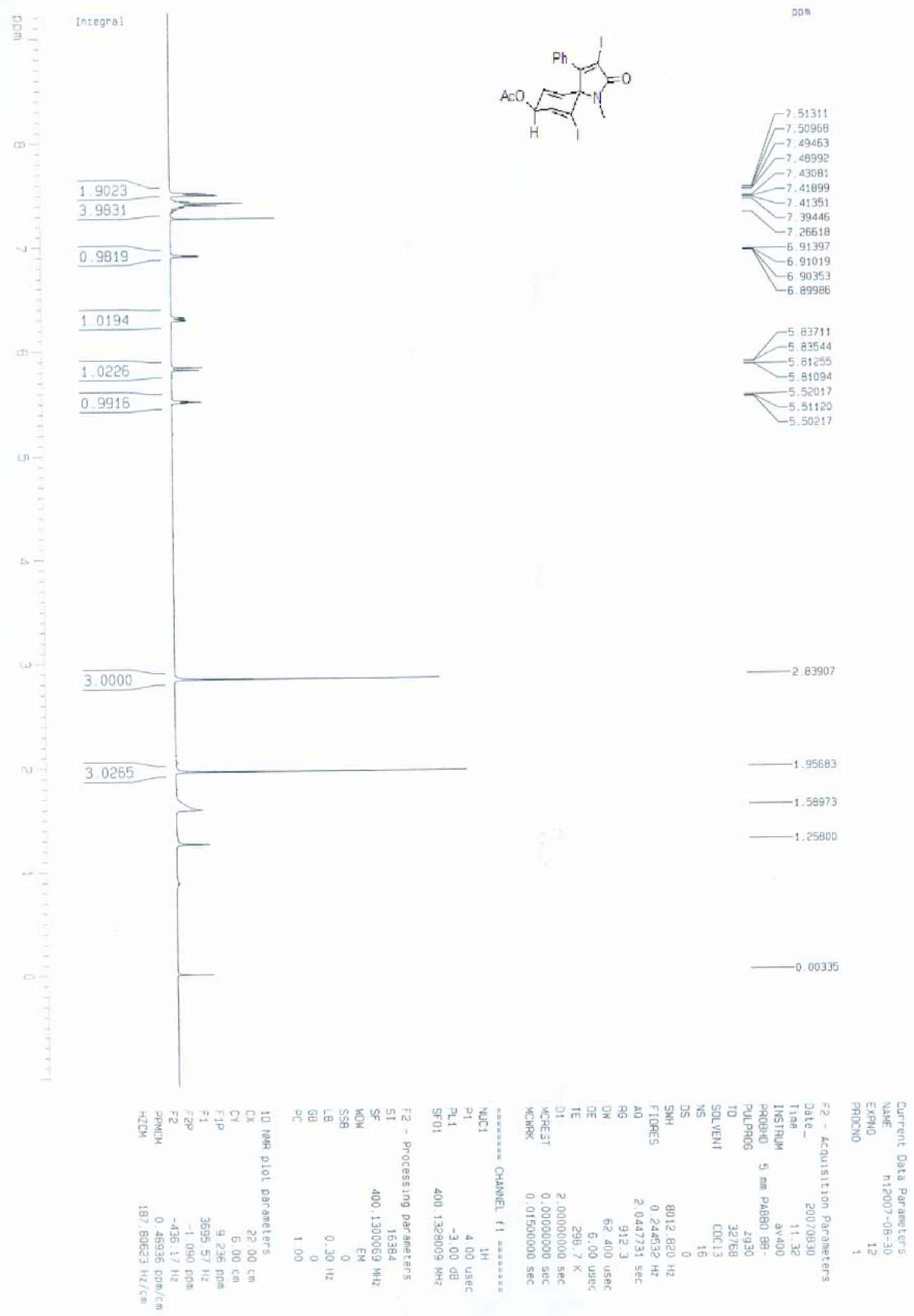


cis-N-methyl-3-iodo-4-(p-methylphenyl)-1-azaspiro[4,5]-deca-3,6,9-trienyl 8-acetate (2m)
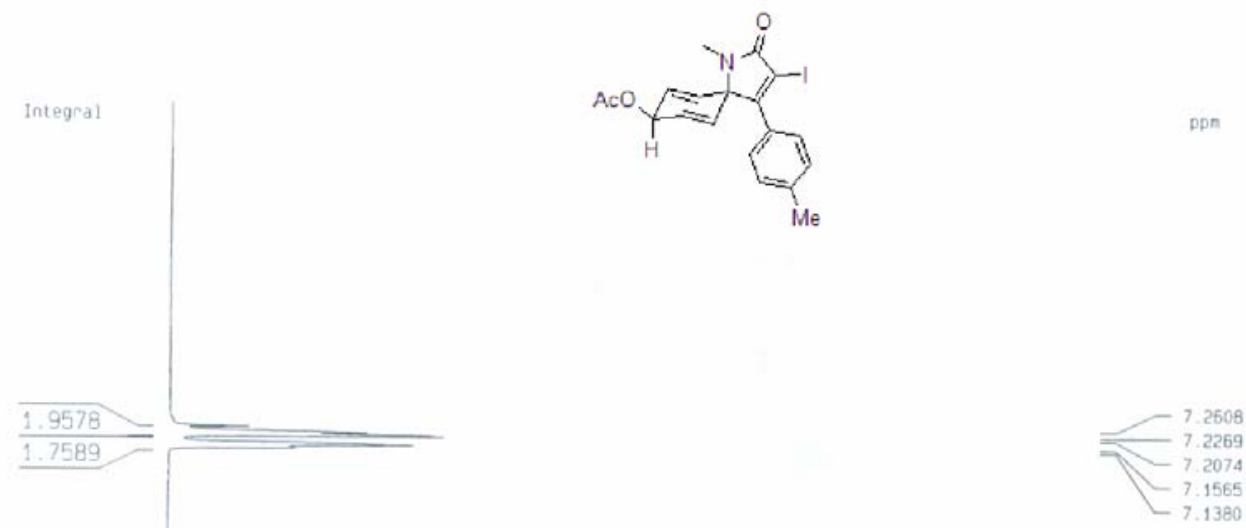

$=-6.154 c$

$=-5.5789$
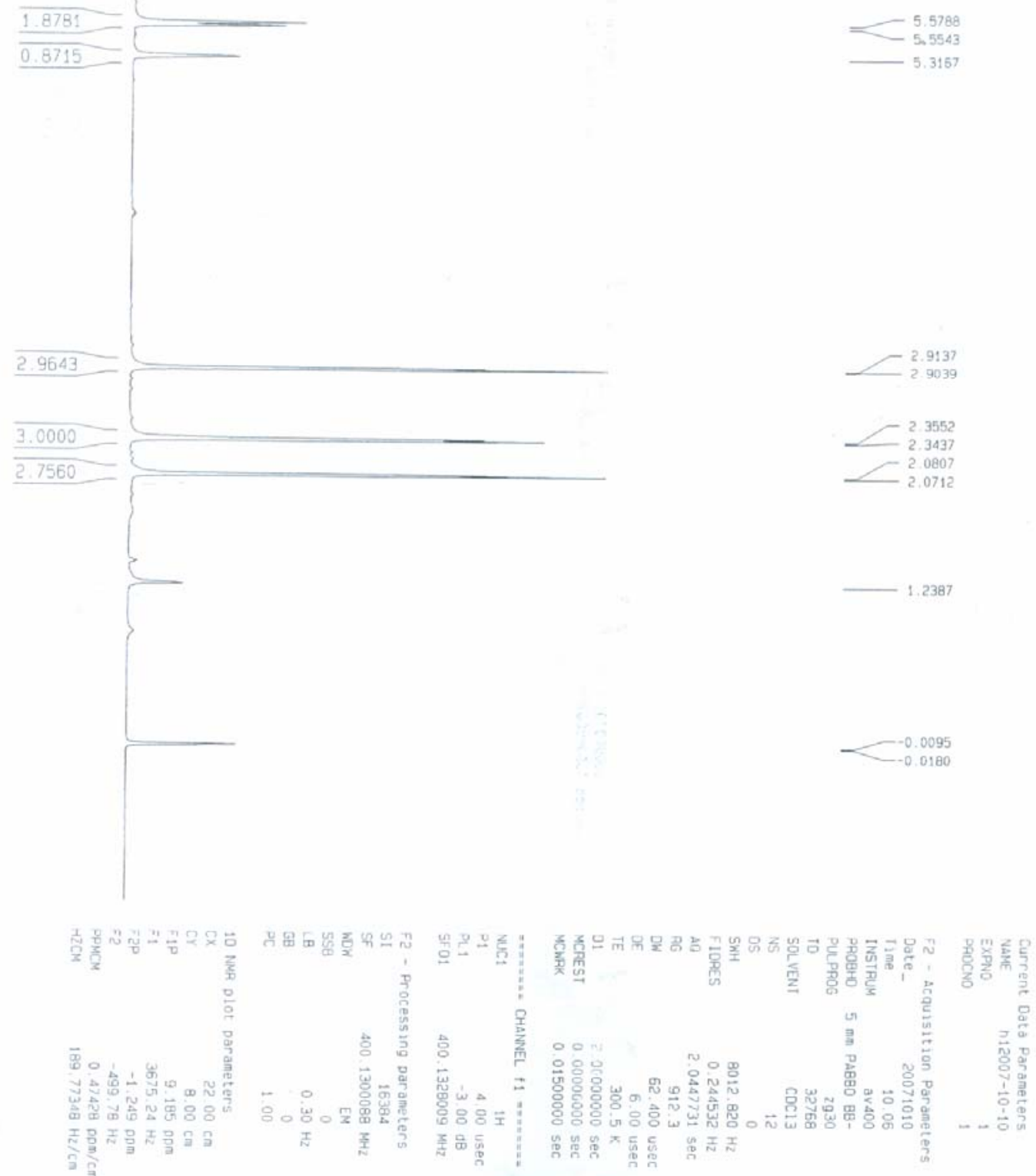
cis-N-methyl-3-iodo-4-(p-methylphenyl)-1-azaspiro[4,5]-deca-3,6,9-trienyl 8-acetate (2m)

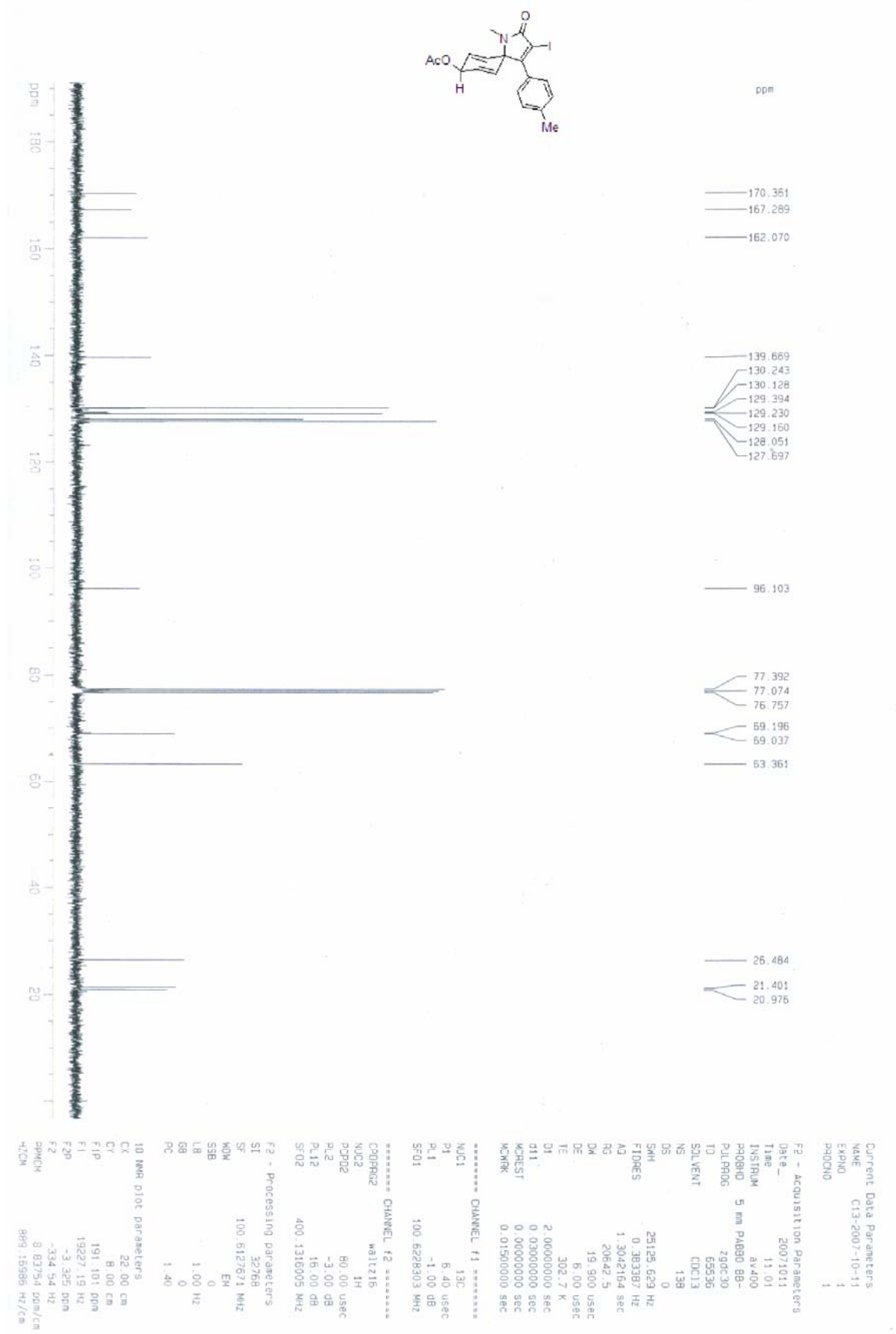


trans-N-methyl-3-iodo-4-(p-methylphenyl)-1-azaspiro[4,5]-deca-3,6,9-trienyl 8-acetate (2m)

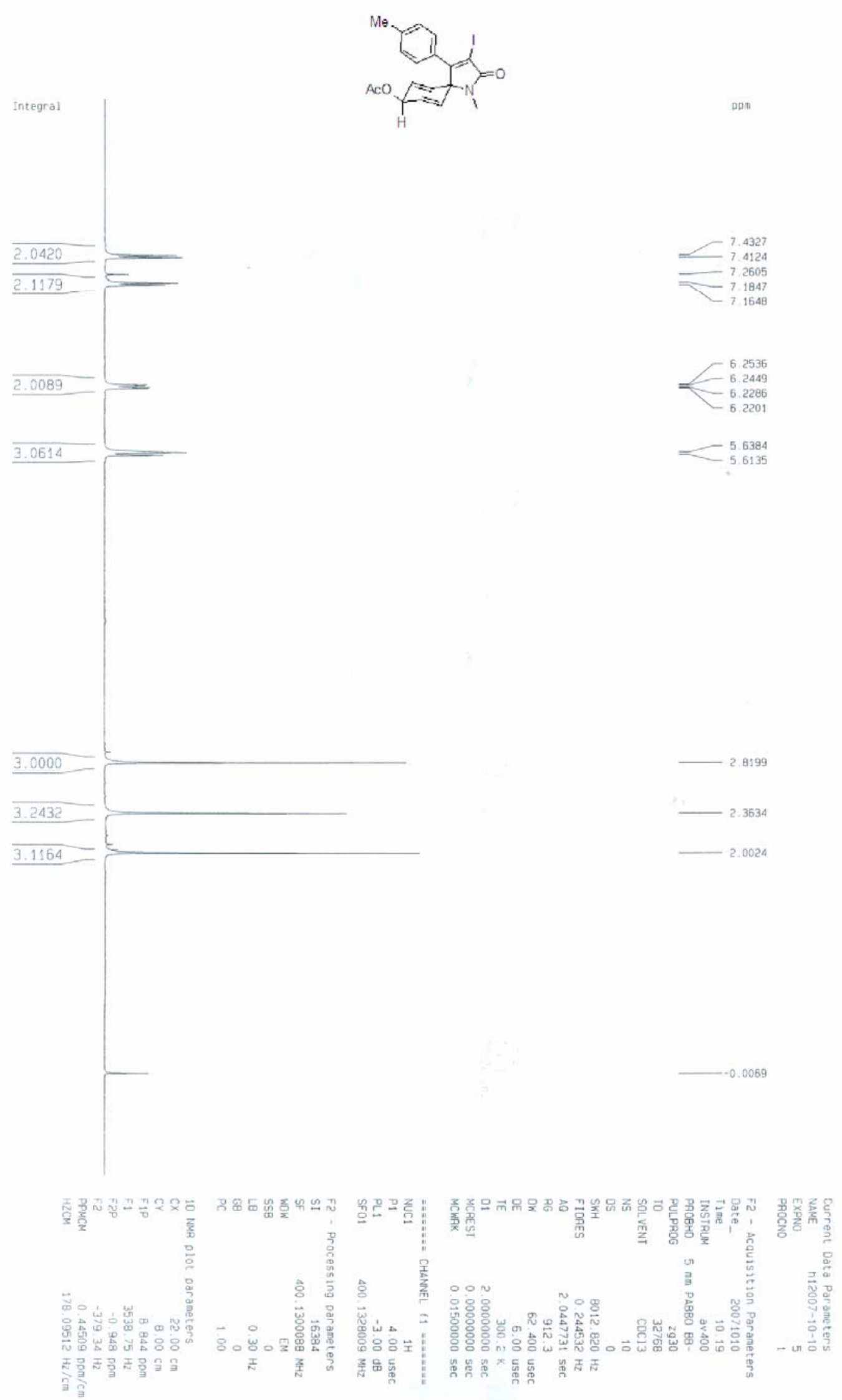


trans-N-methyl-3-iodo-4-(p-methylphenyl)-1-azaspiro[4,5]-deca-3,6,9-trienyl 8-acetate (2m)

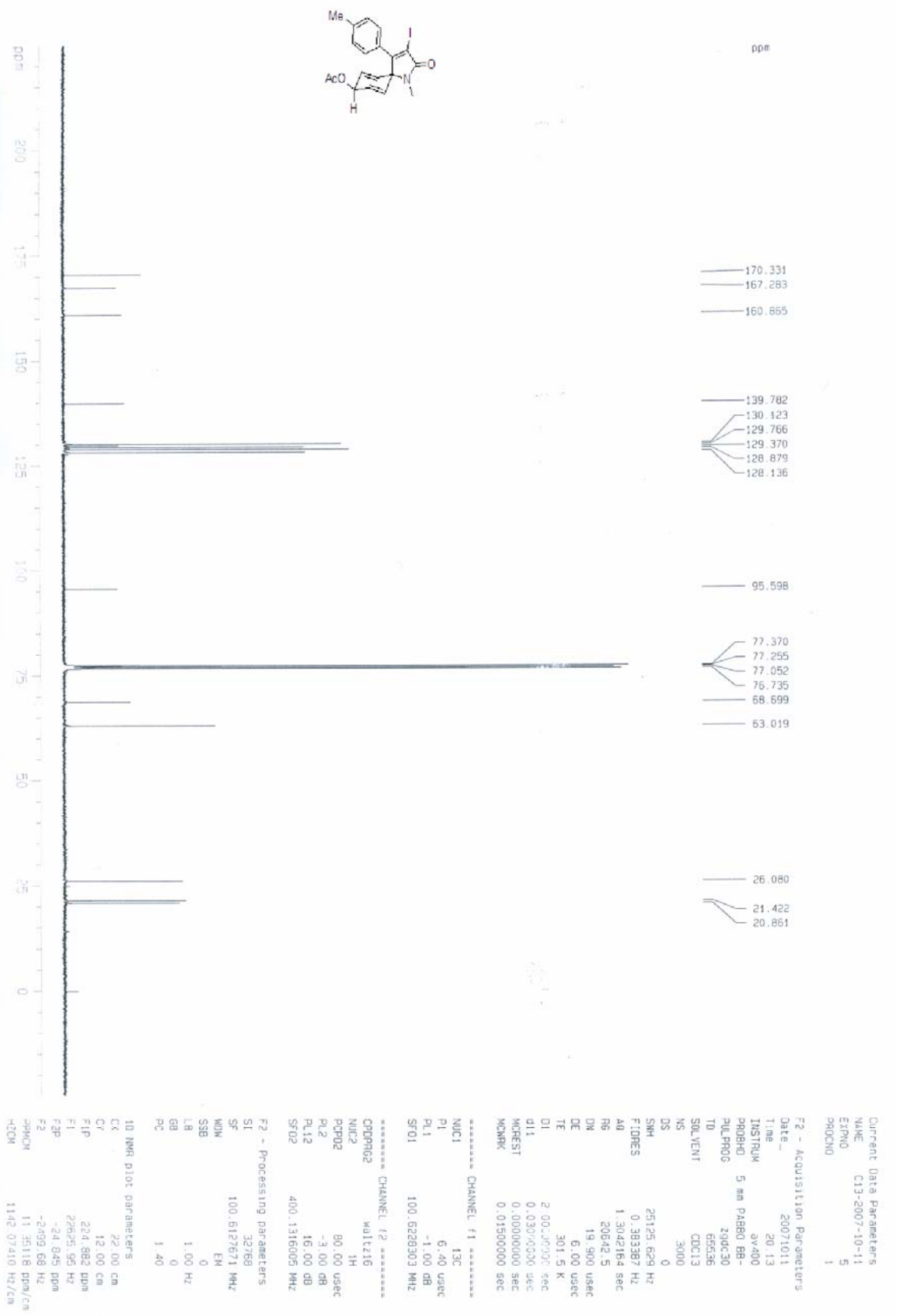


cis-N-methyl-3-iodo-4-(p-methoxyphenyl)-1-azaspiro[4,5]-deca-3,6,9-trienyl 8-acetate (2n)
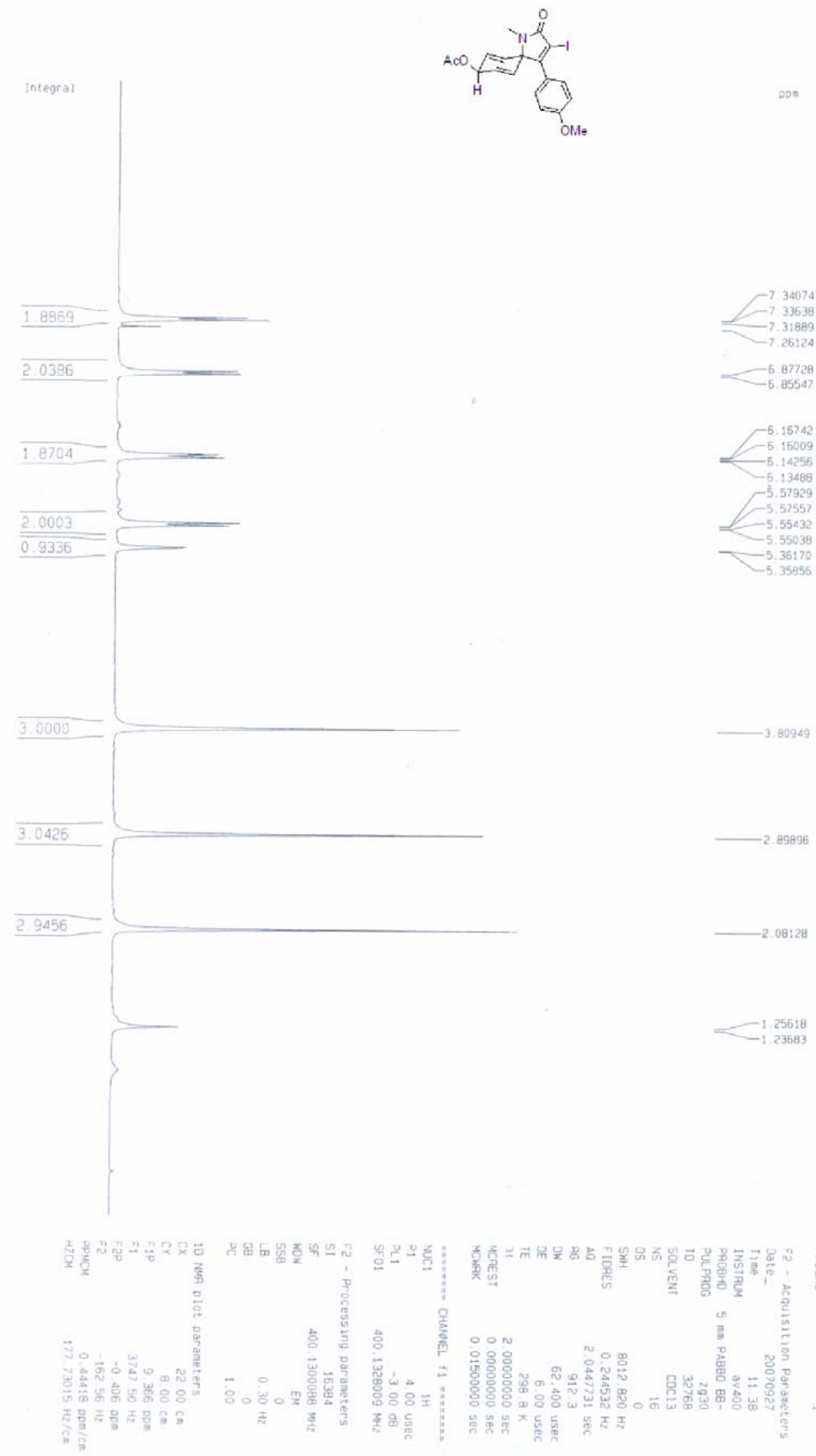
cis-N-methyl-3-iodo-4-(p-methoxyphenyl)-1-azaspiro[4,5]-deca-3,6,9-trienyl 8-acetate (2n)

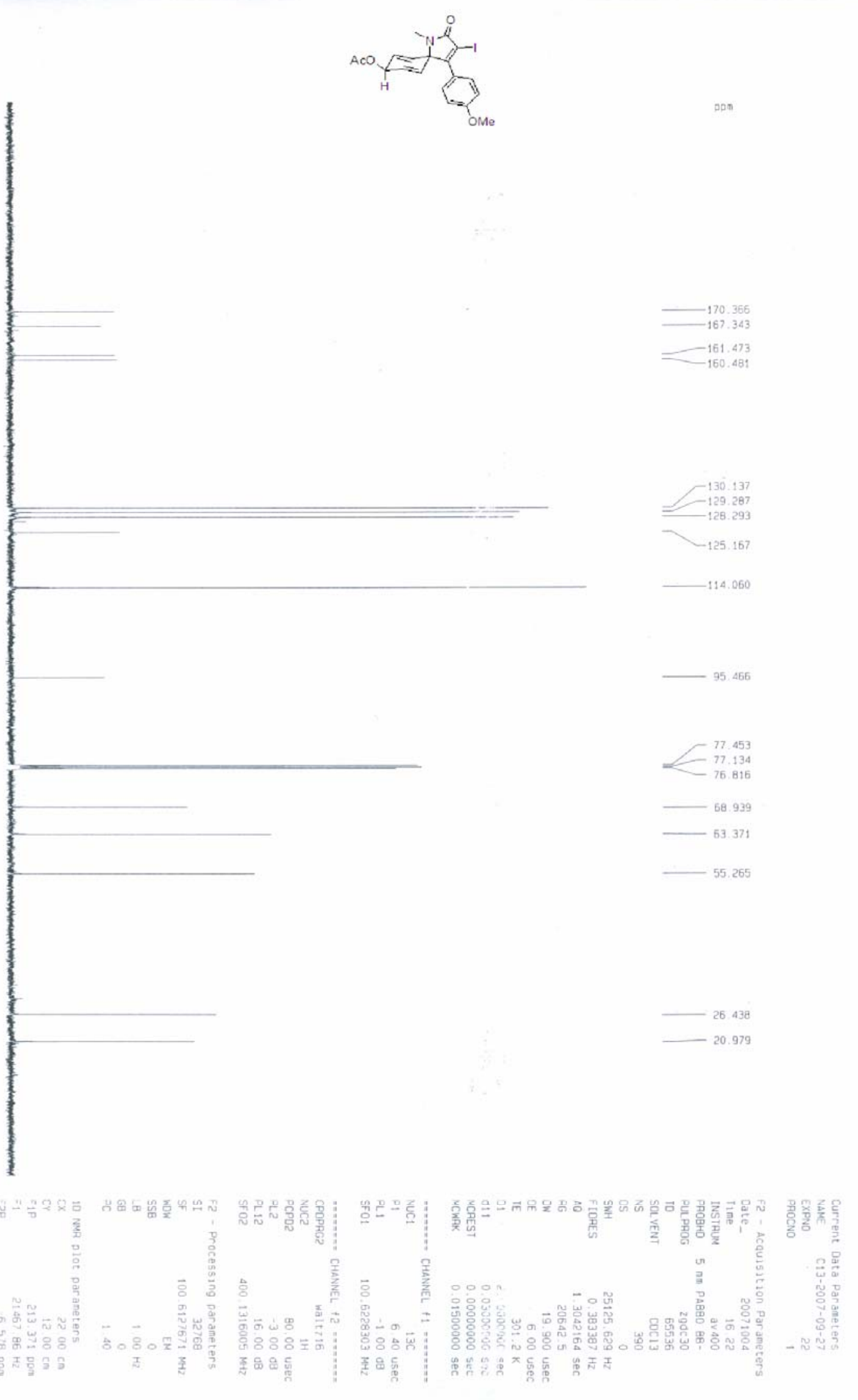


trans-N-methyl-3-iodo-4-(p-methoxyphenyl)-1-azaspiro[4,5]-deca-3,6,9-trienyl 8-acetate (2n)
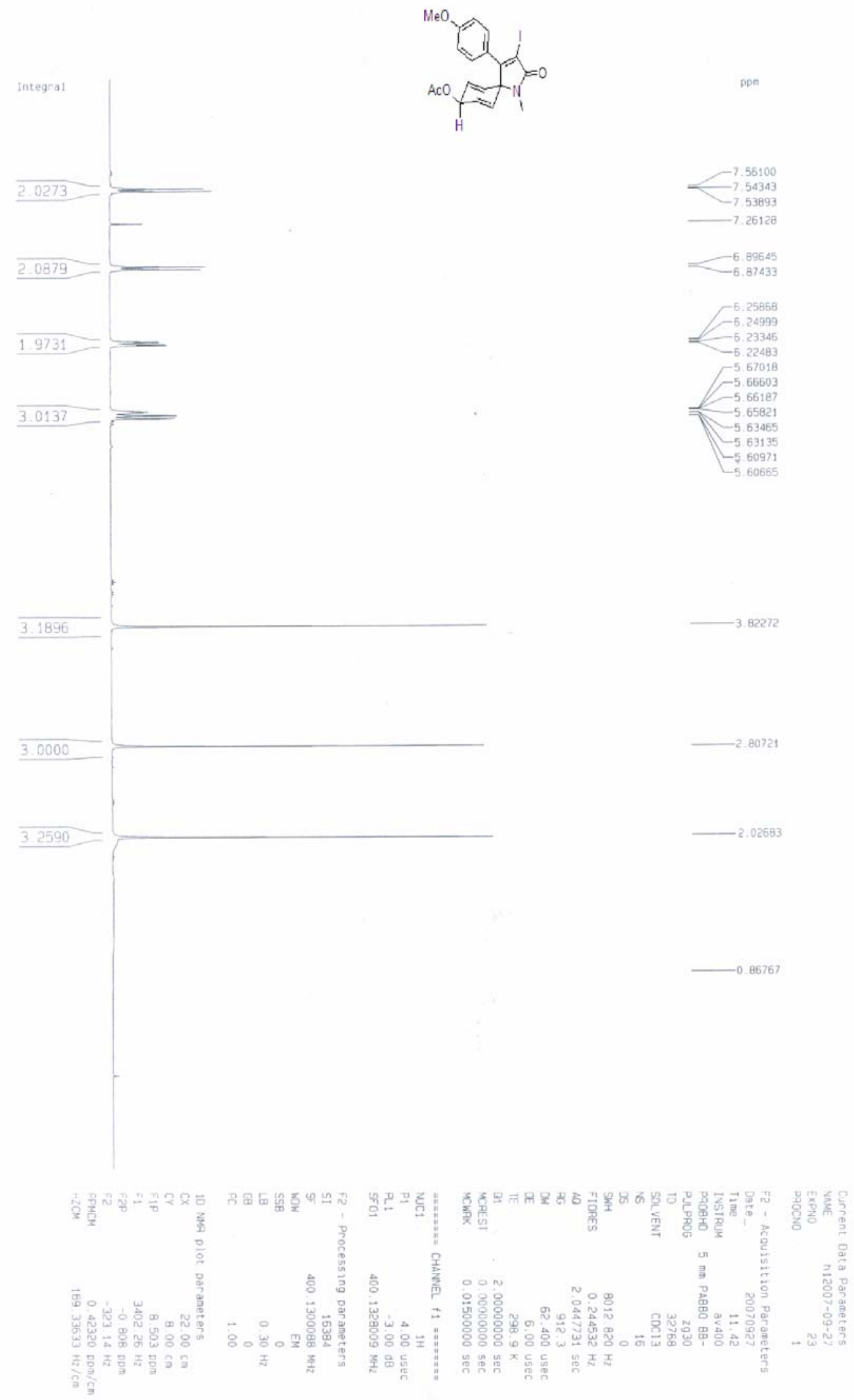
trans-N-methyl-3-iodo-4-(p-methoxyphenyl)-1-azaspiro[4,5]-deca-3,6,9-trienyl 8-acetate (2n)

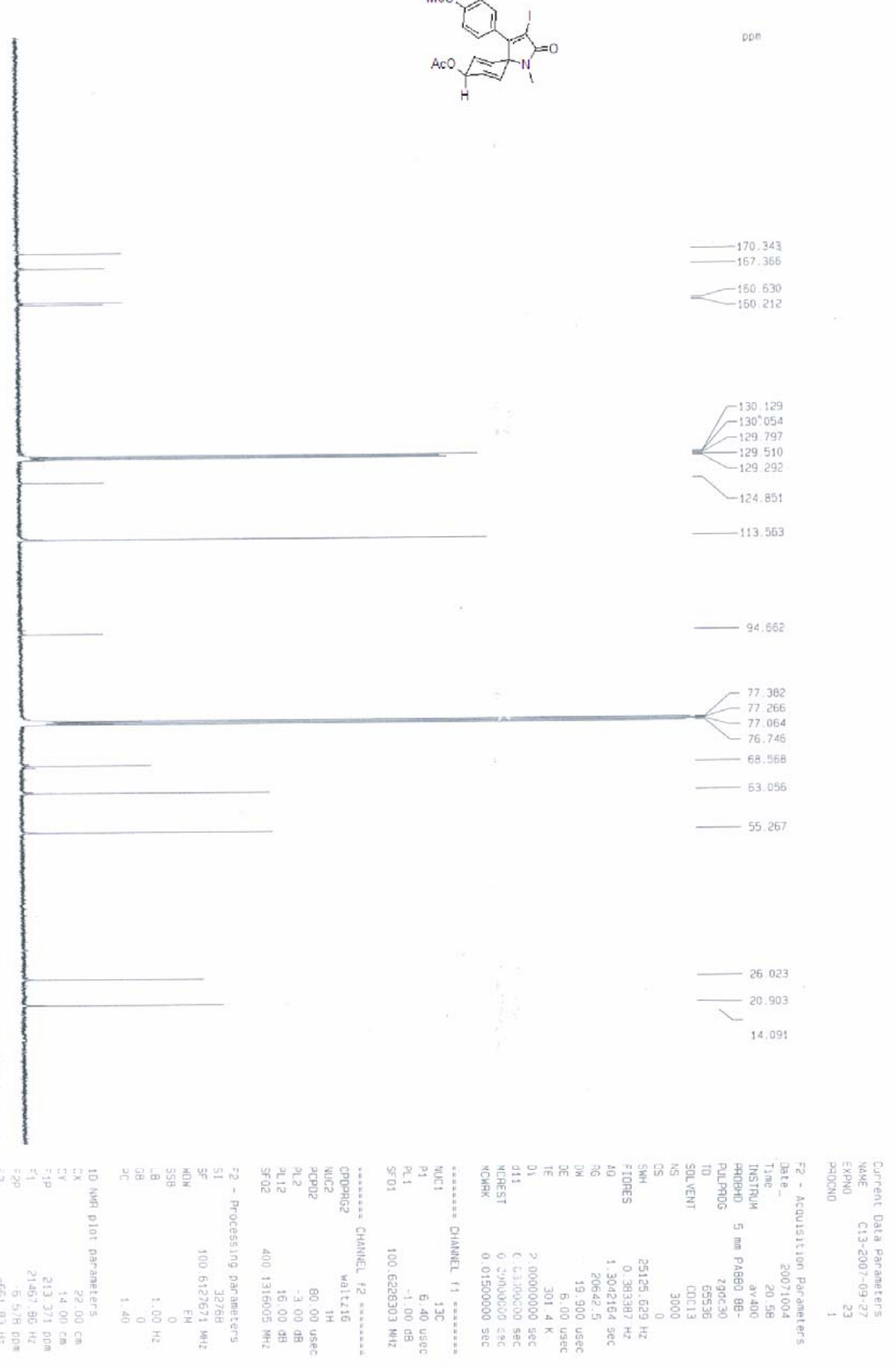


cis-N-methyl-3-iodo-4-methyl-1-azaspiro[4,5]-deca-3,6,9-trienyl 8-acetate (2p)

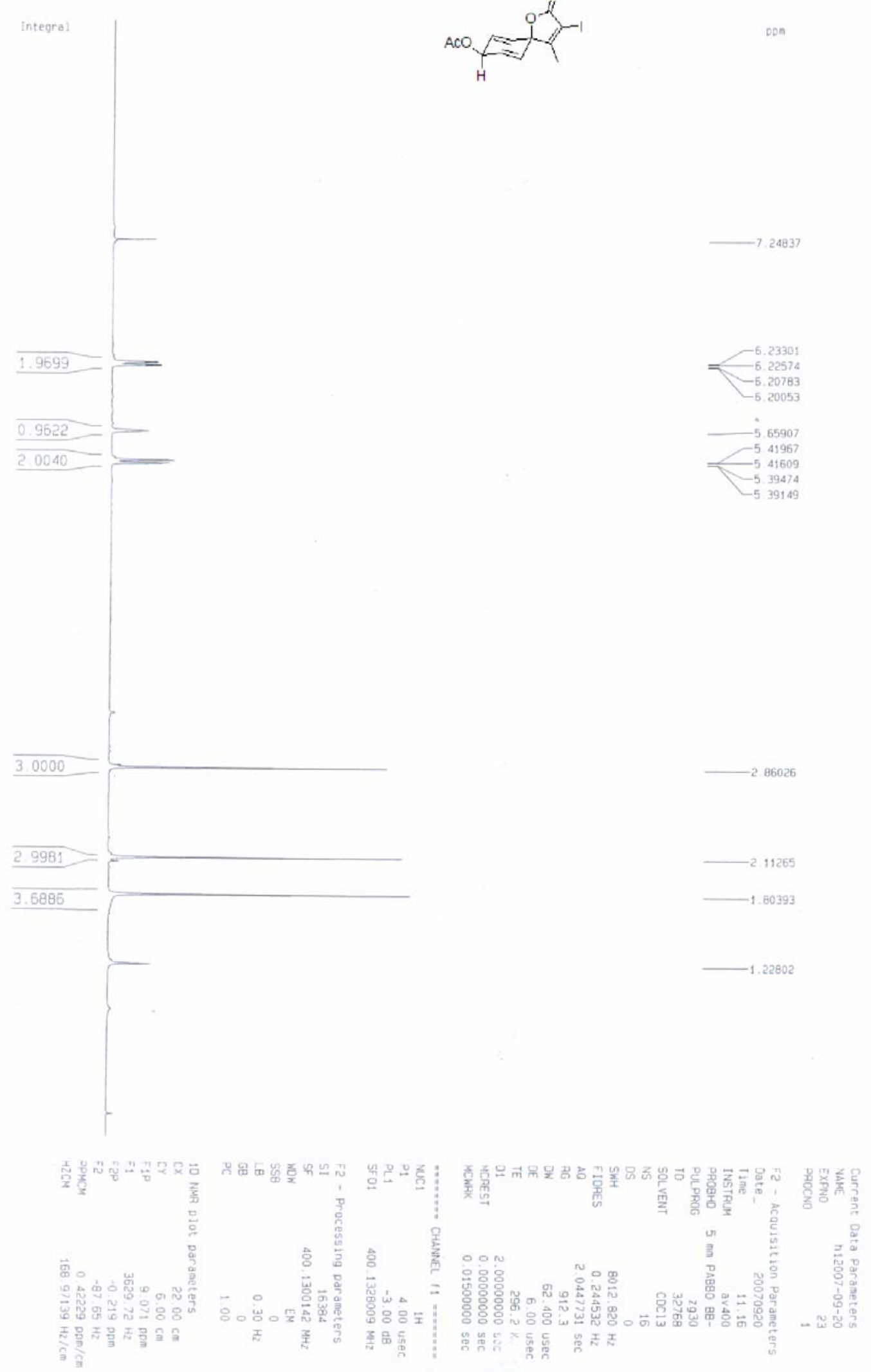


cis-N-methyl-3-iodo-4-methyl-1-azaspiro[4,5]-deca-3,6,9-trienyl 8-acetate (2p)

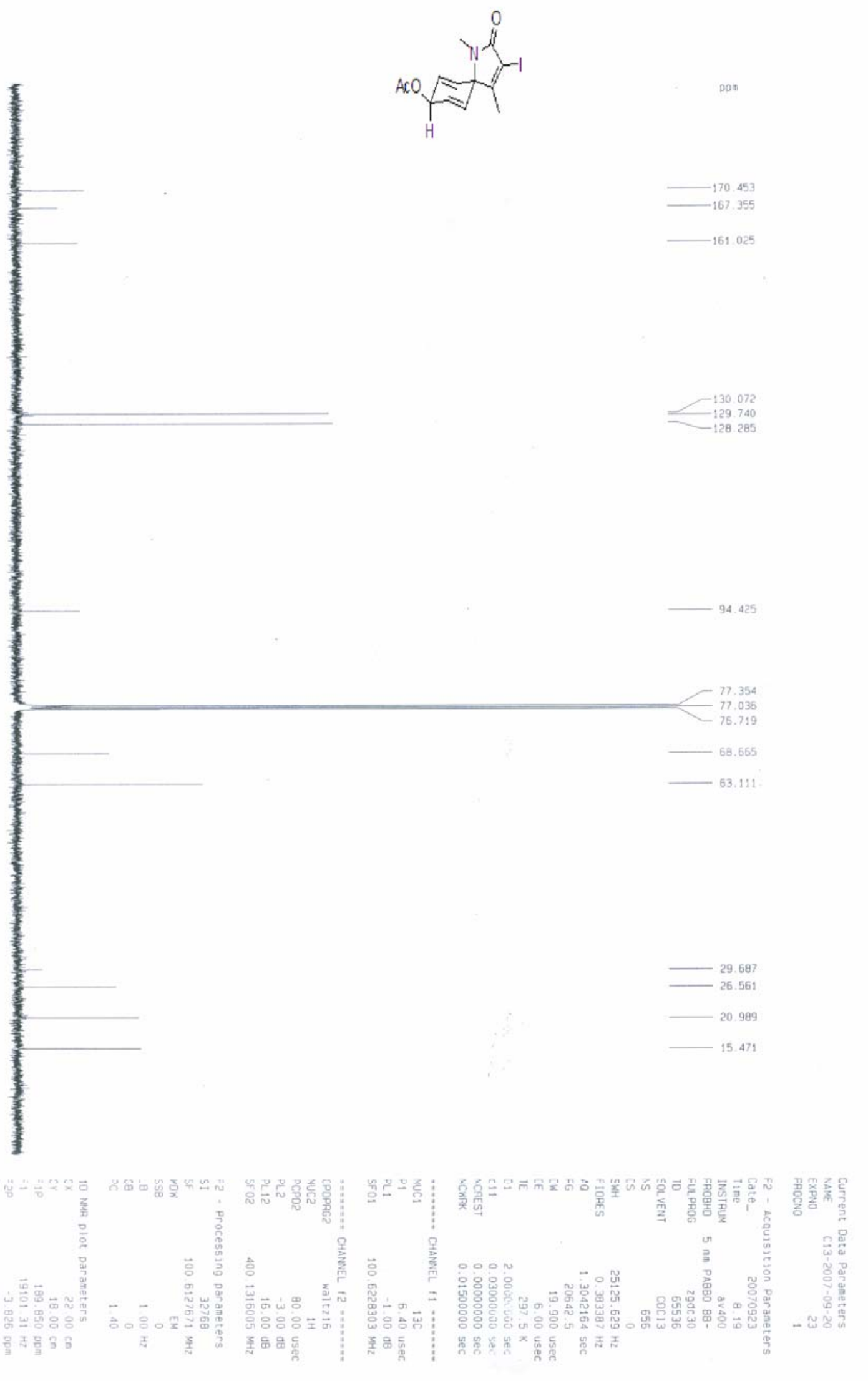


trans-N-methyl-3-iodo-4-methyl-1-azaspiro[4,5]-deca-3,6,9-trienyl 8-acetate (2p)

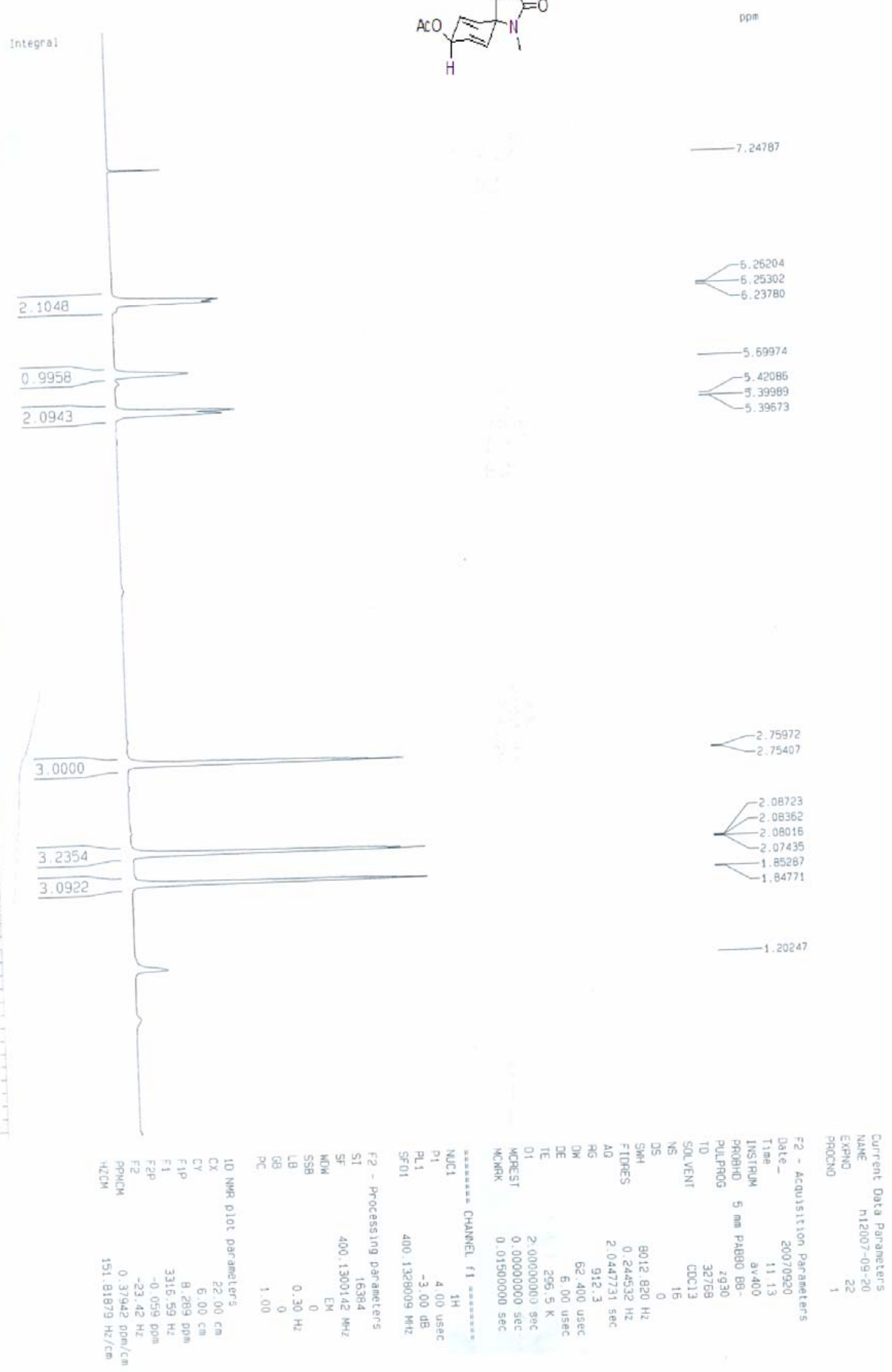


trans-N-methyl-3-iodo-4-methyl-1-azaspiro[4,5]-deca-3,6,9-trienyl 8-acetate (2p)
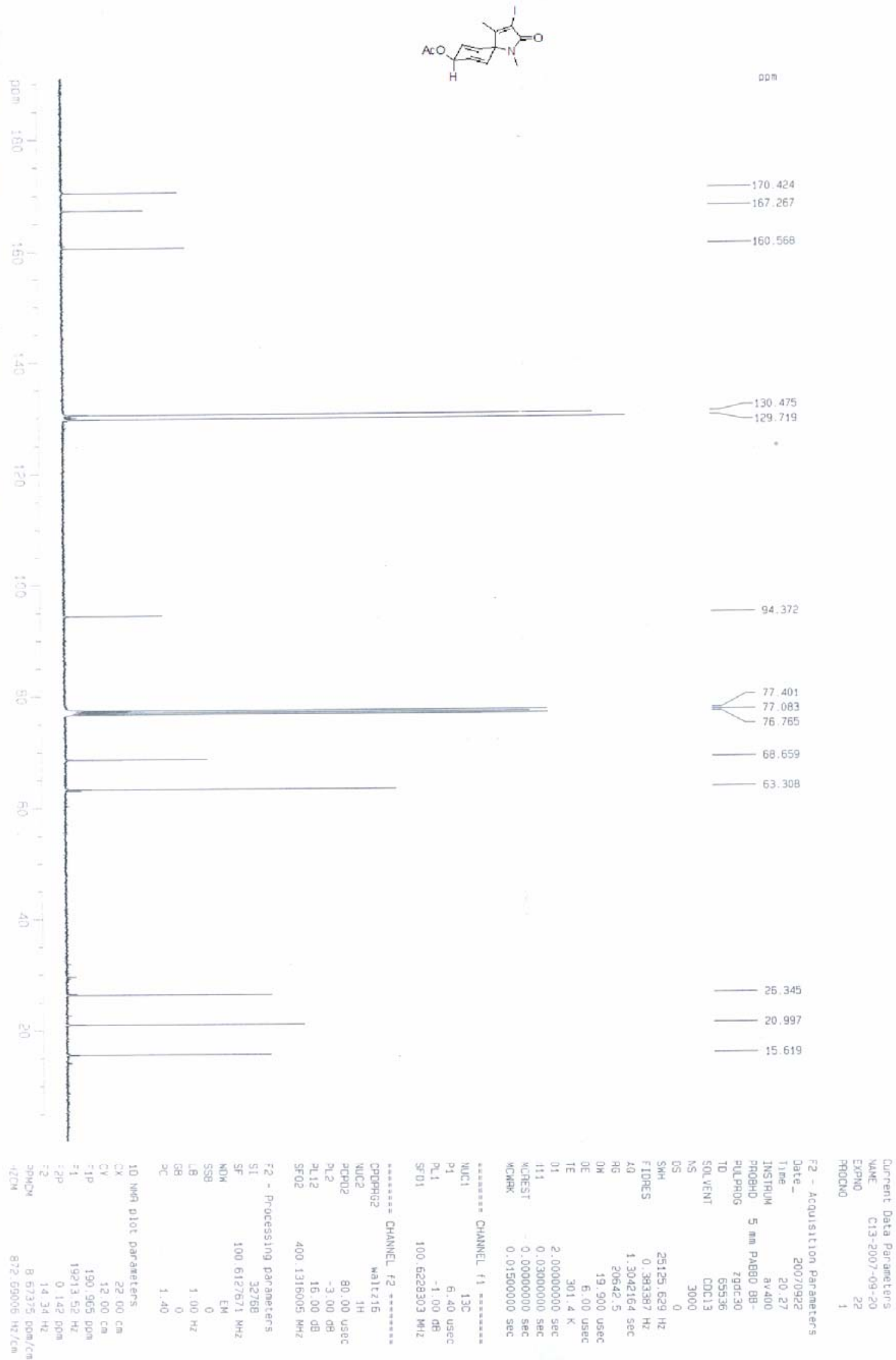
cis-3-Iodo-4-phenyl-1-oxaspiro[4,5]-deca-3,6,9-trienyl 8-acetate (2t)

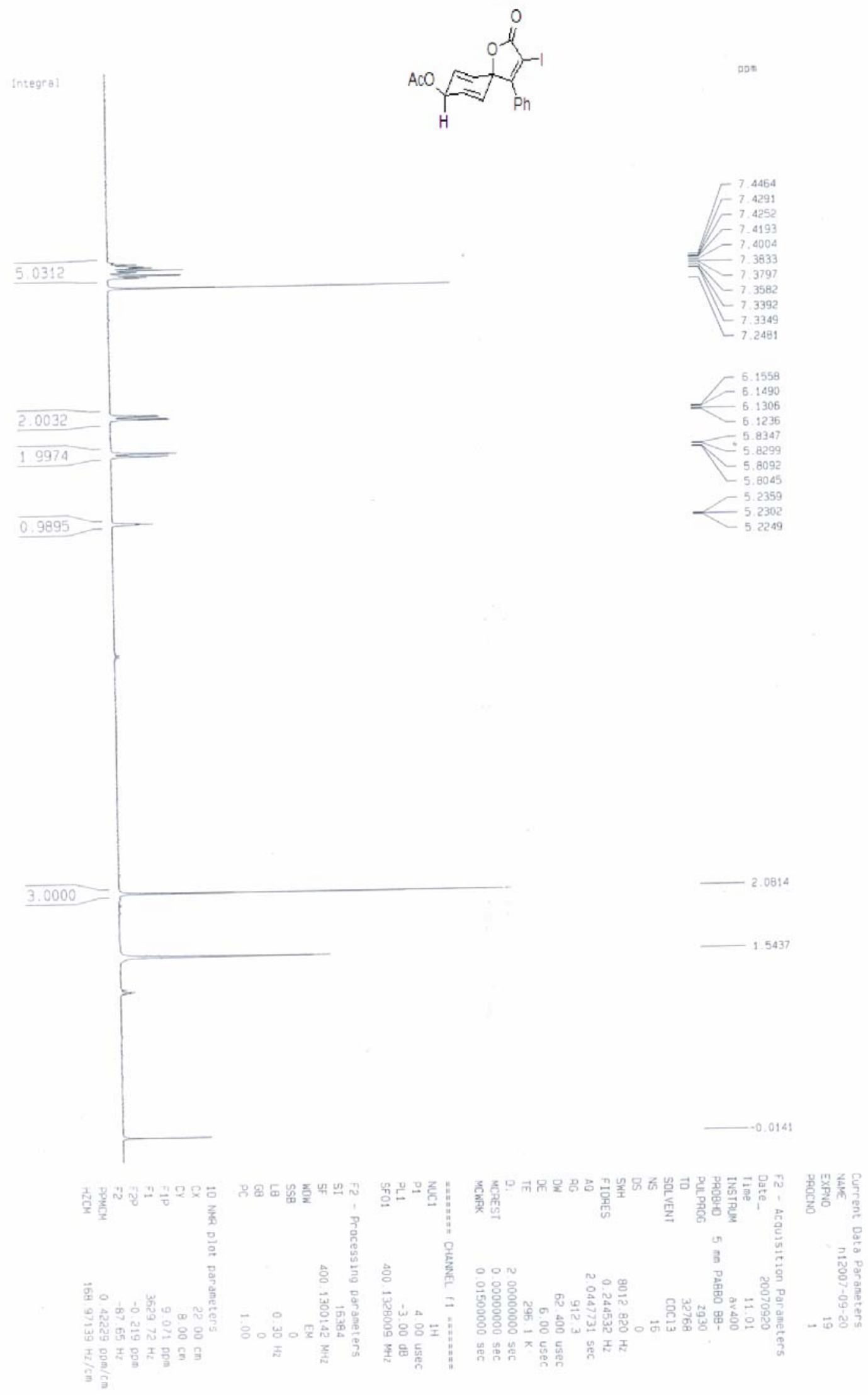


cis-3-Iodo-4-phenyl-1-oxaspiro[4,5]-deca-3,6,9-trienyl 8-acetate (2t)
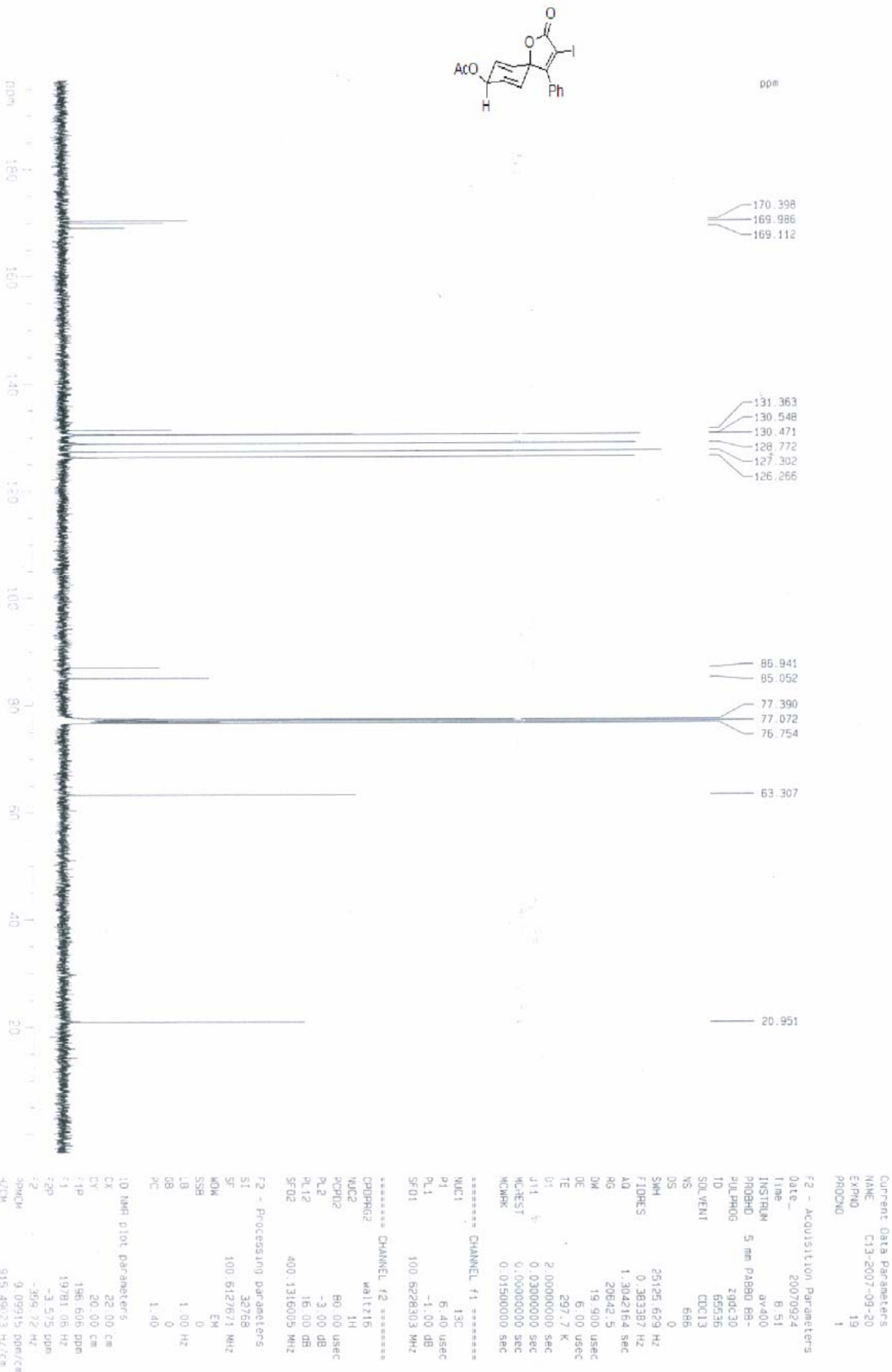
trans-3-Iodo-4-phenyl-1-oxaspiro[4,5]-deca-3,6,9-trienyl 8-acetate (2t)

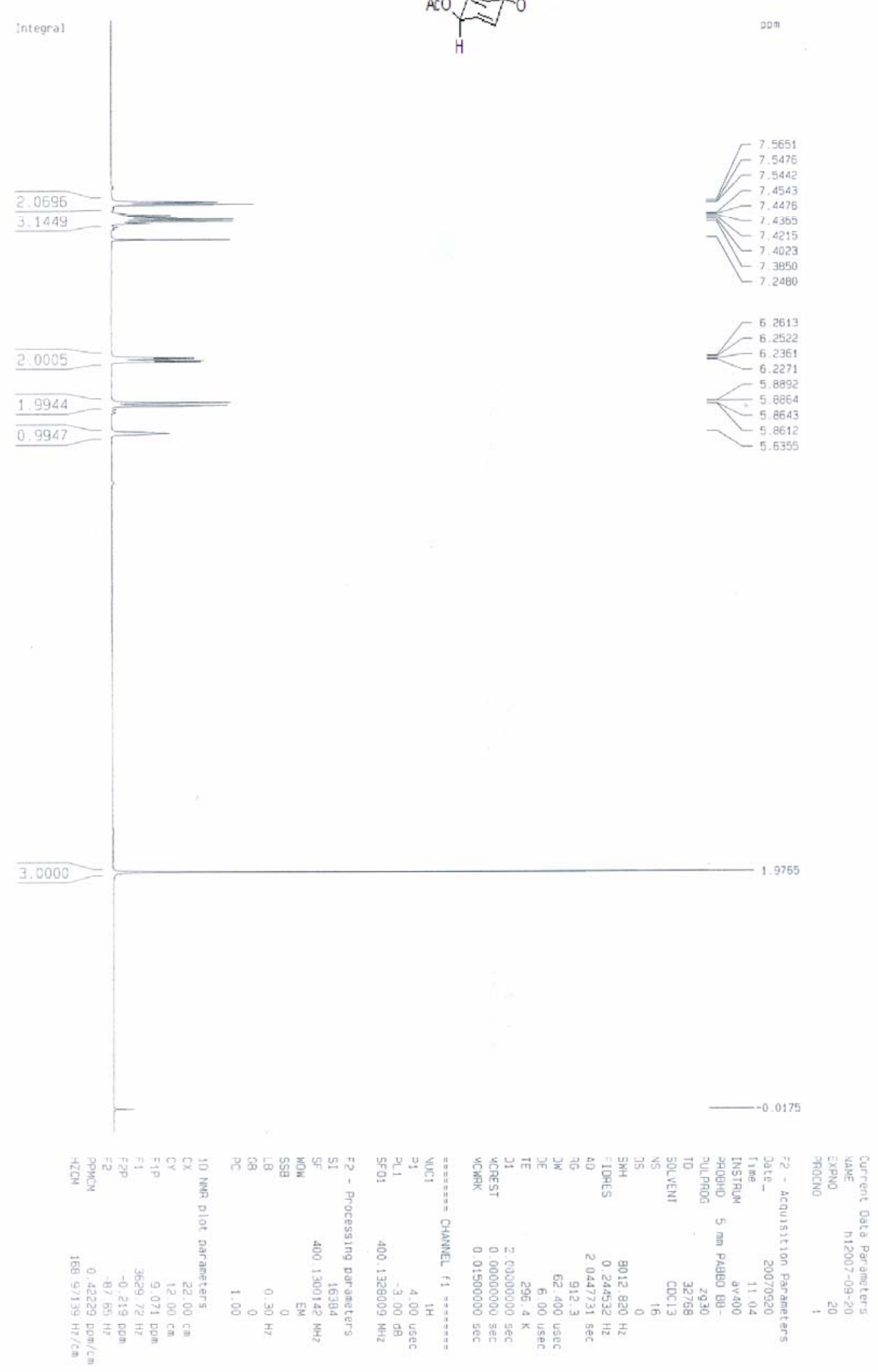


trans-3-Iodo-4-phenyl-1-oxaspiro[4,5]-deca-3,6,9-trienyl 8-acetate (2t)
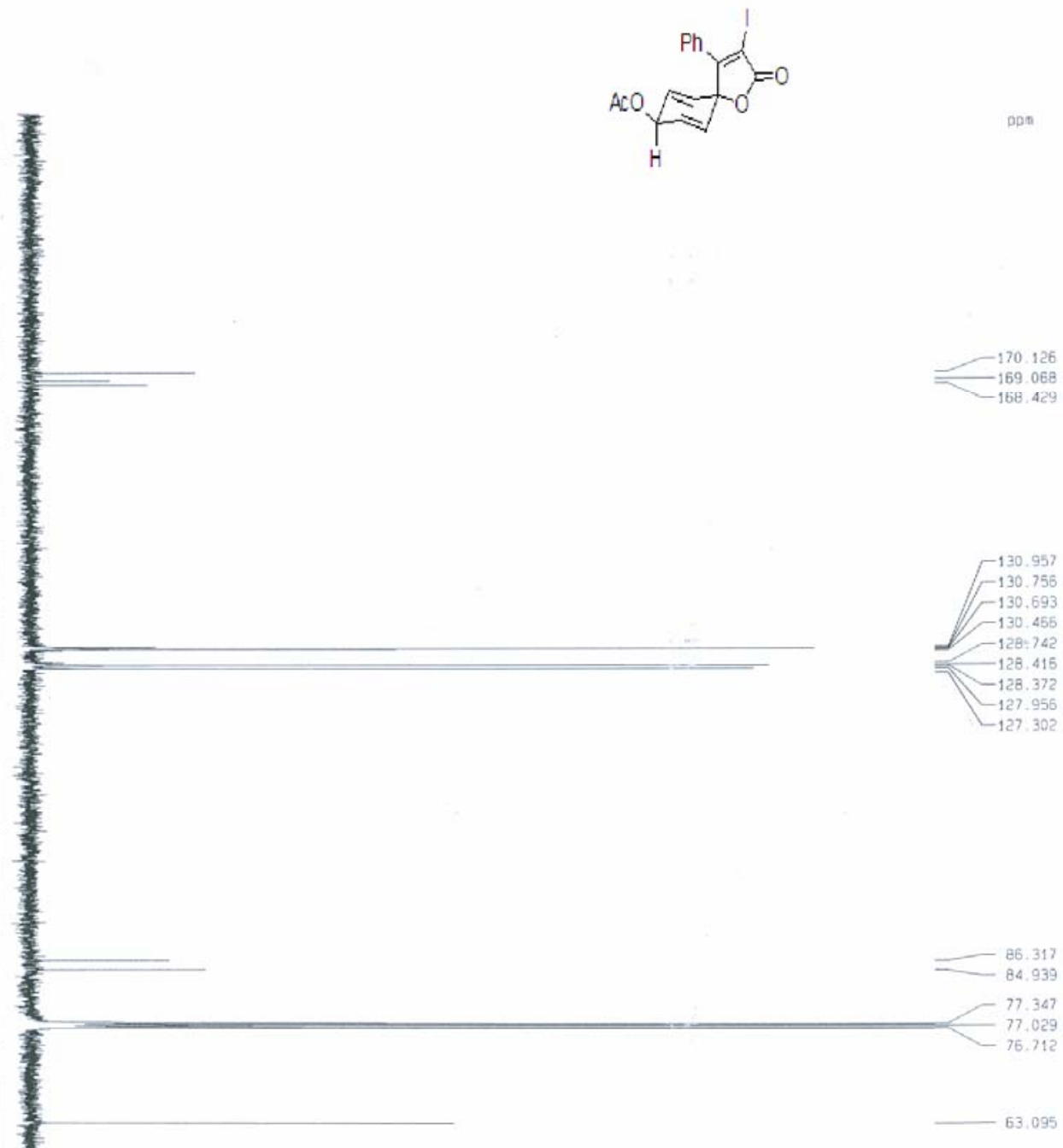

$\frac{1}{4}$

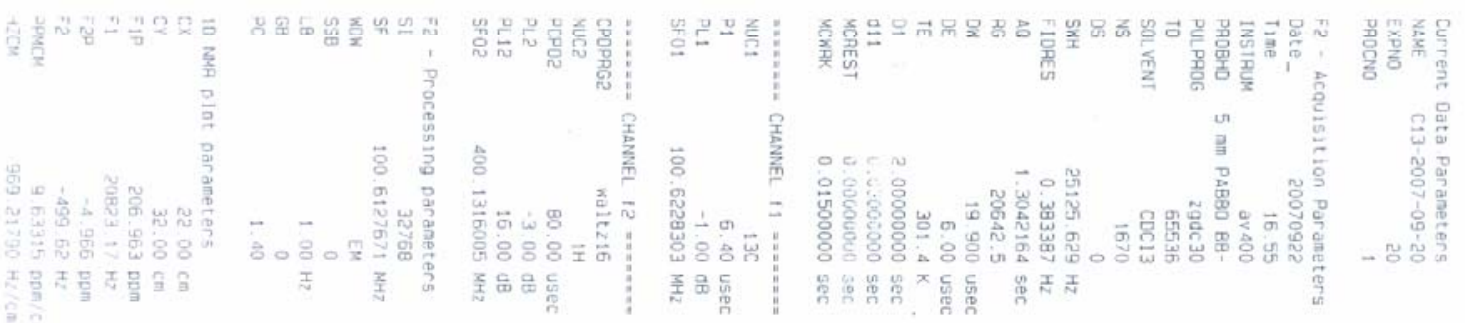


N-Methyl-3-iodo-4-phenyl-1-azaspiro[4,5]-deca-3,6,8-trien-10-one (3r)

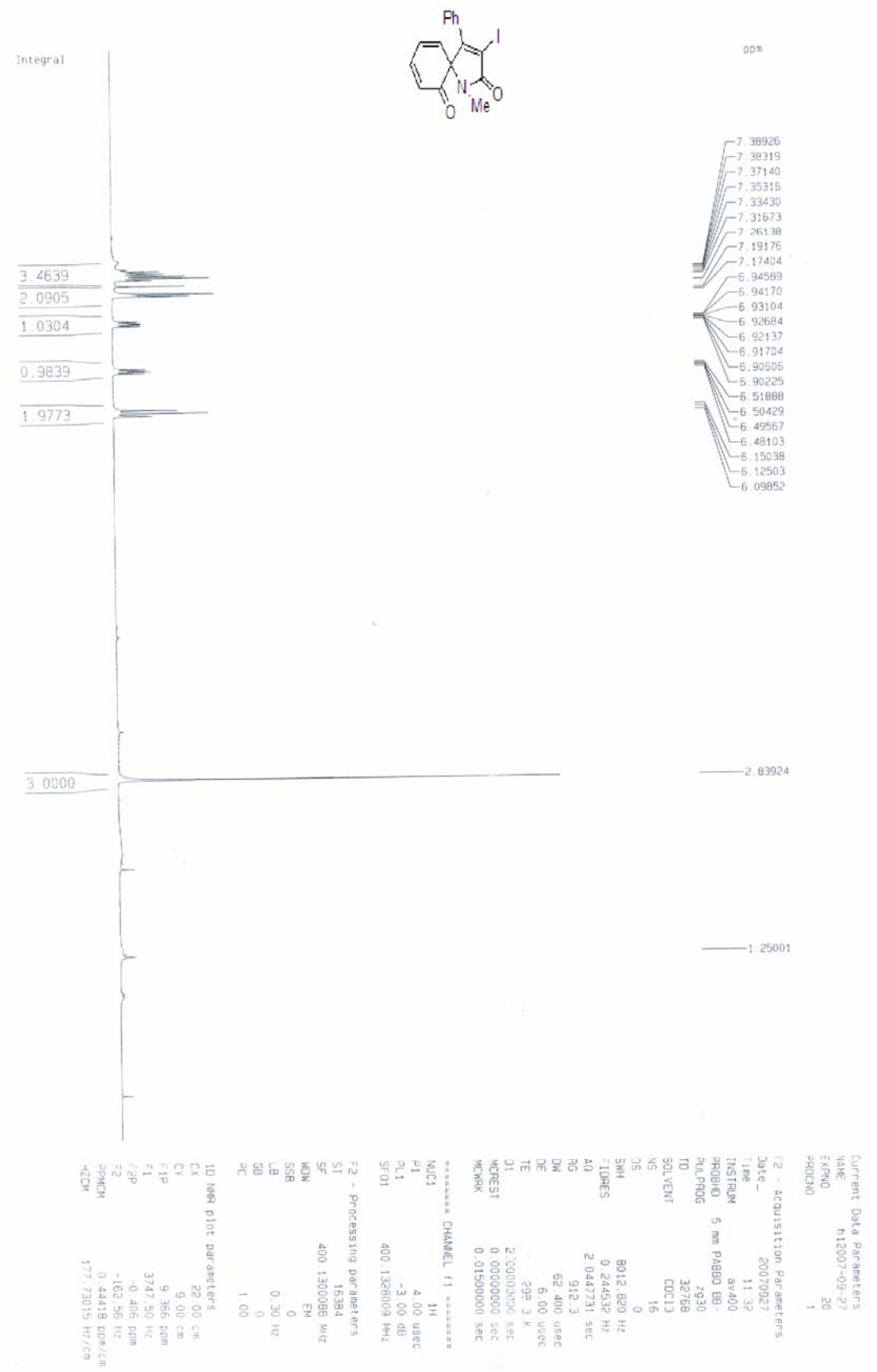


N-Methyl-3-iodo-4-phenyl-1-azaspiro[4,5]-deca-3,6,8-trien-10-one (3r)
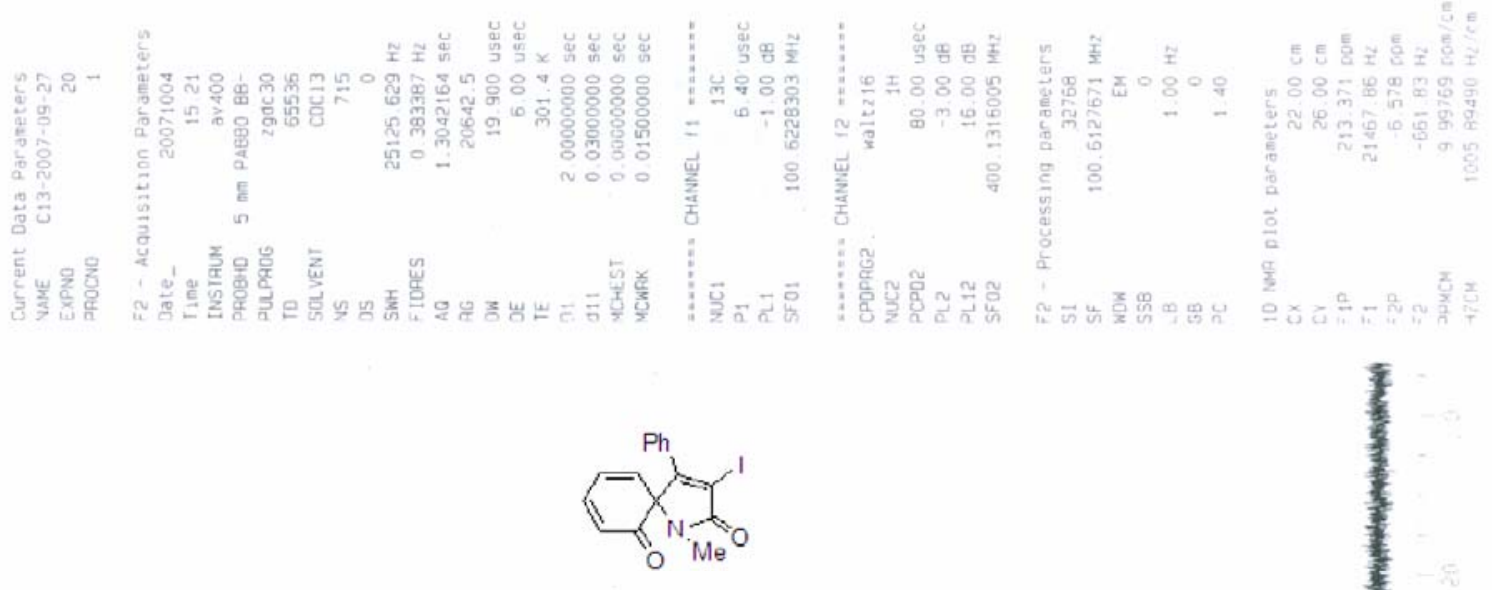

$005: 22$
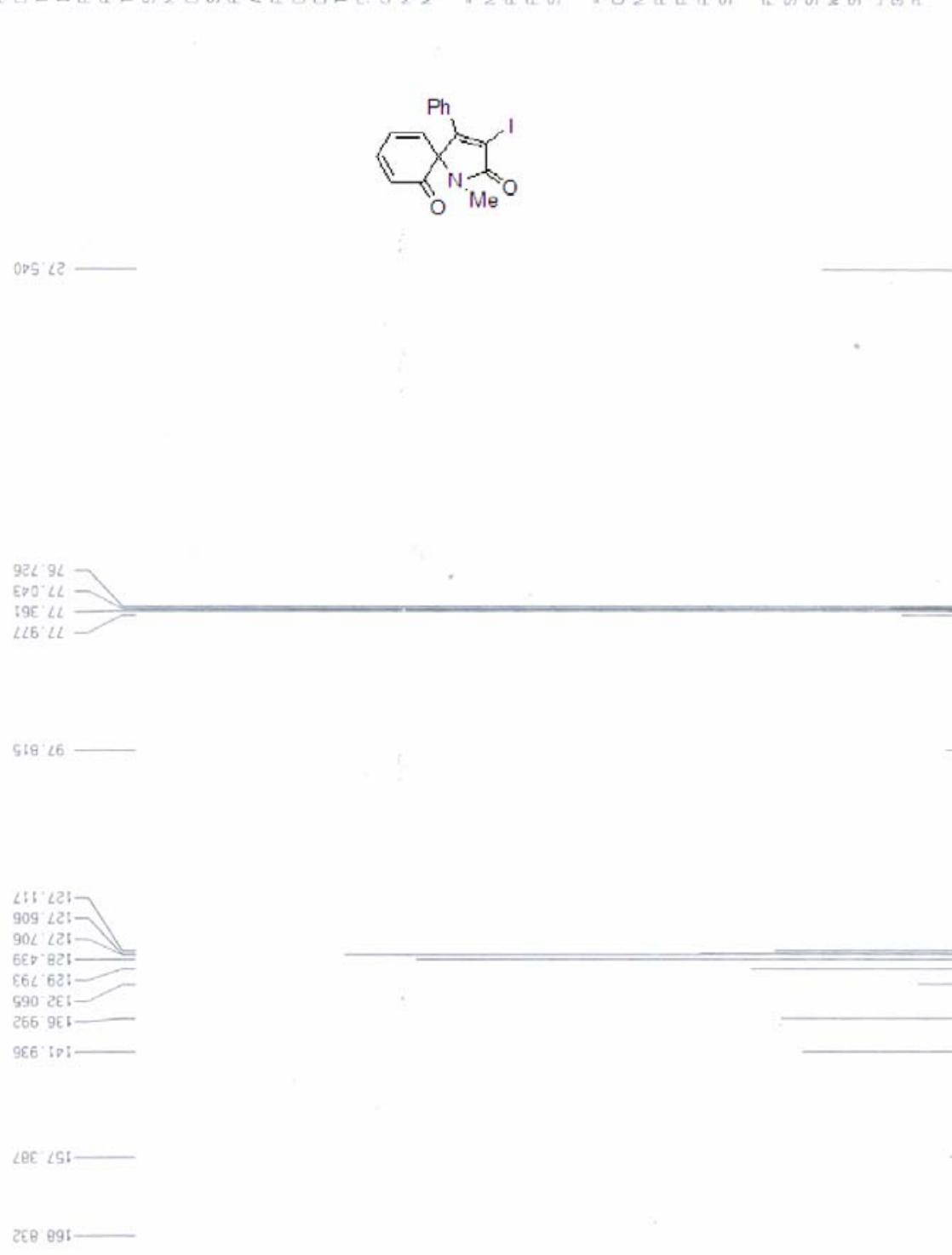
N-Methyl-3-iodo-4-phenyl-1-azaspiro[4,5]-deca-3,6,9-trien-8-one (3u)

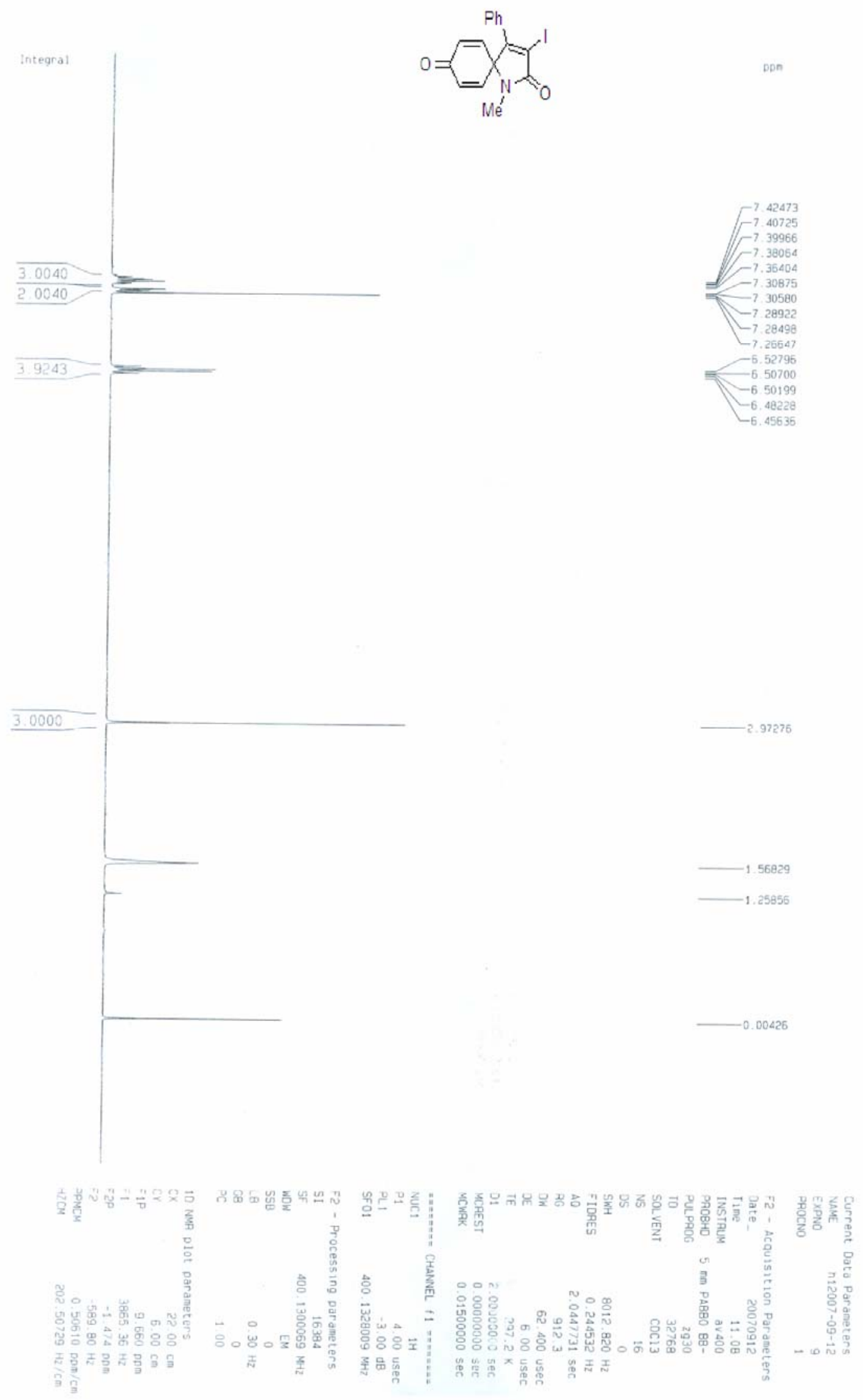


N-Methyl-3-iodo-4-phenyl-1-azaspiro[4,5]-deca-3,6,9-trien-8-one (3u)

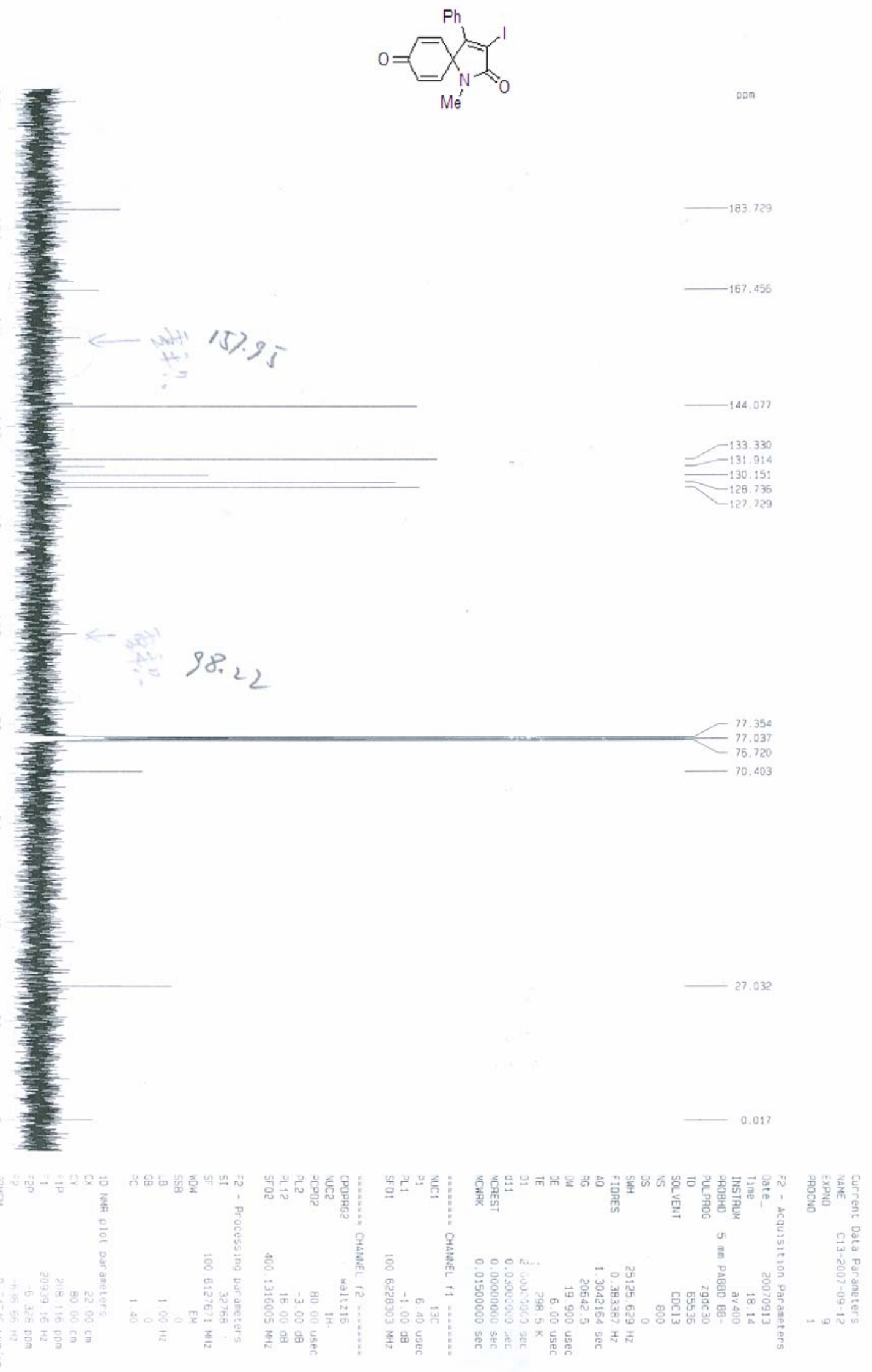


cis-N-Methyl-3-iodo-4-phenyl-6-chloro-1-azaspiro[4,5]-deca-3,6,9-trienyl 8-acetate (2i)<smiles>CC(=O)O[C@H]1C=C[C@]2(C(Cl)=C1)C(c1ccccc1)=C(I)C(=O)N2C</smiles>
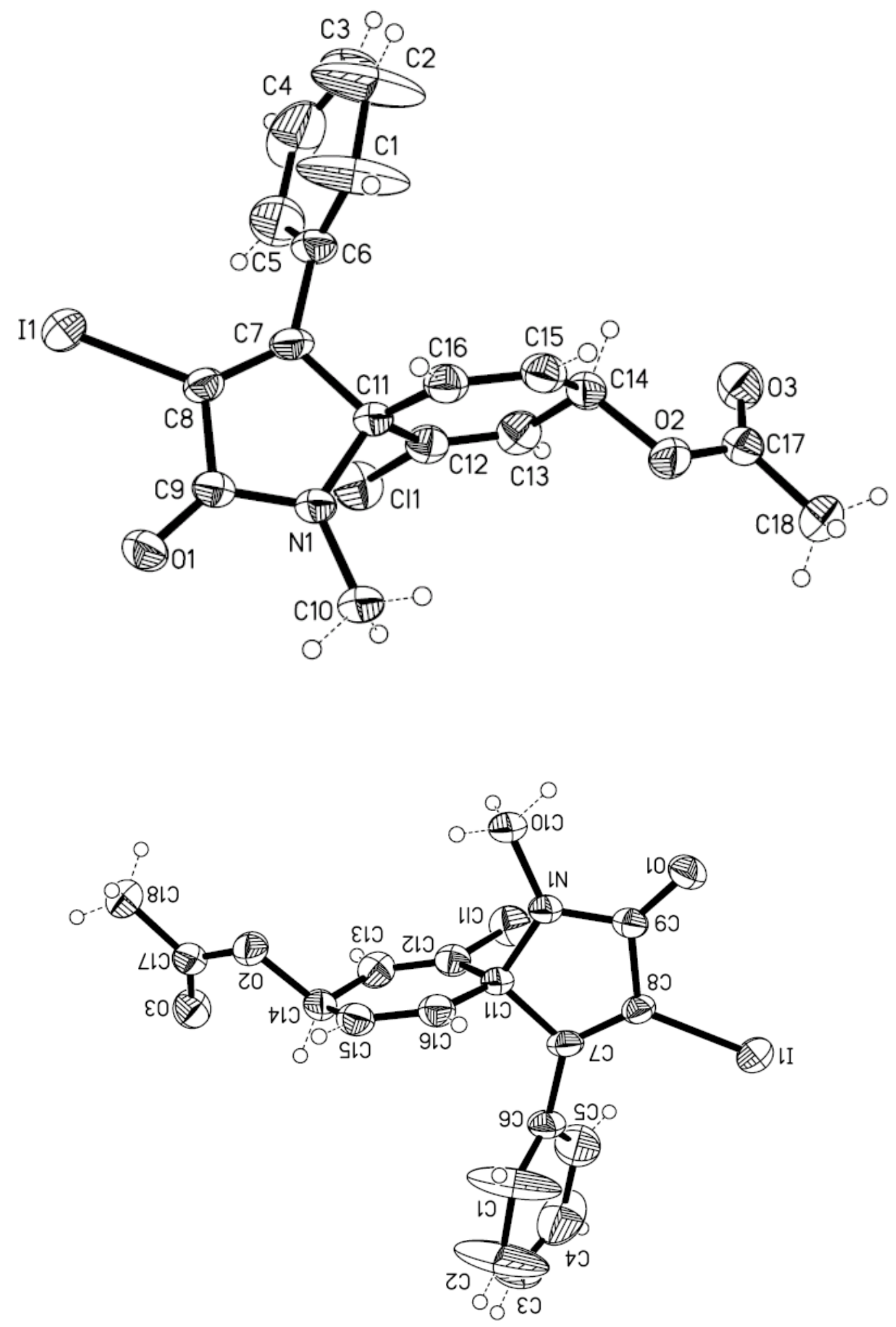
Table 1. Crystal data and structure refinement for JH.

Identification code jh

Empirical formula

Formula weight

Temperature

Wavelength
C18 H15 CI I N O3

455.66

298(2) K

0.71073 A

Crystal system, space group $\quad$ Monoclinic, P 21/c

Unit cell dimensions $\quad \mathrm{a}=\mathbf{1 4 . 8 6 0 ( 2 )} \mathrm{A}$ alpha $=90 \mathrm{deg}$.

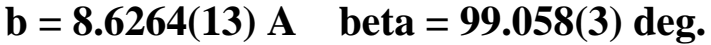
$c=14.429(2) \mathrm{A}$ gamma $=90 \mathrm{deg}$.

Volume

Z, Calculated density

Absorption coefficient

$\mathbf{F}(\mathbf{0 0 0})$
1826.6(5) $A^{\wedge} 3$

4, $1.657 \mathrm{Mg} / \mathrm{m} \wedge 3$

$1.914 \mathrm{~mm}^{\wedge}-1$

896

Crystal size

$0.45 \times 0.31$ x $0.25 \mathrm{~mm}$

Theta range for data collection 2.74 to 25.02 deg.

Limiting indices $-16<=h<=17,-10<=k<=10,-17<=l<=8$

Reflections collected / unique 9265 / $3203[R($ int $)=0.0183]$

$$
\text { Completeness to theta }=25.02 \quad 99.4 \%
$$

Absorption correction Semi-empirical from equivalents

Max. and min. transmission $\quad 0.6461$ and 0.4796

Refinement method $\quad$ Full-matrix least-squares on $F^{\wedge} 2$

Data / restraints / parameters 3203 / 0 / 219

$$
\text { Goodness-of-fit on } F^{\wedge} 2
$$

Final $R$ indices $[\mathrm{I}>2 \operatorname{sigma}(\mathrm{I})] \quad \mathrm{R} 1=\mathbf{0 . 0 5 4 1}, \mathrm{wR} 2=\mathbf{0 . 1 5 4 8}$

$\mathrm{R}$ indices (all data) $\quad \mathrm{R} 1=0.0592, \mathrm{wR} 2=0.1596$

Largest diff. peak and hole $\quad 1.698$ and -1.024 e.A^^-3 
Table 2. Atomic coordinates $\left(x 1^{\wedge} 4\right)$ and equivalent isotropic displacement parameters $(A \wedge 2 \times 10 \wedge 3)$ for $\mathrm{JH}$.

$\mathrm{U}(\mathrm{eq})$ is defined as one third of the trace of the orthogonalized Uij tensor.

\begin{tabular}{lcccc}
\hline & & & & \\
& & X & U(eq) \\
I(1) & $6432(1)$ & $10490(1)$ & $5166(1)$ & $71(1)$ \\
Cl(1) & $7845(1)$ & $5174(2)$ & $5756(1)$ & $81(1)$ \\
O(1) & $8687(3)$ & $9991(6)$ & $5384(3)$ & $66(1)$ \\
O(2) & $8827(3)$ & $2750(5)$ & $2996(3)$ & $66(1)$ \\
O(3) & $8010(4)$ & $547(5)$ & $2940(4)$ & $76(1)$ \\
N(1) & $8598(3)$ & $7671(5)$ & $4588(3)$ & $49(1)$ \\
C(1) & $5820(8)$ & $7000(20)$ & $2891(10)$ & $206(9)$ \\
C(2) & $4926(10)$ & $6510(30)$ & $2542(12)$ & $245(12)$ \\
C(3) & $4364(6)$ & $5994(16)$ & $3032(9)$ & $130(4)$ \\
C(4) & $4588(7)$ & $6276(14)$ & $3957(13)$ & $150(6)$ \\
C(5) & $5471(6)$ & $6793(11)$ & $4338(8)$ & $106(3)$ \\
C(6) & $6102(4)$ & $6976(8)$ & $3809(4)$ & $61(1)$ \\
C(7) & $7021(3)$ & $7552(7)$ & $4207(4)$ & $52(1)$ \\
C(8) & $7252(3)$ & $8824(7)$ & $4703(4)$ & $49(1)$ \\
C(9) & $8251(3)$ & $8955(7)$ & $4950(4)$ & $49(1)$ \\
C(11) & $7893(3)$ & $6655(6)$ & $4102(4)$ & $48(1)$ \\
C(10) & $9558(4)$ & $7290(8)$ & $4727(4)$ & $62(1)$ \\
C(12) & $7921(4)$ & $5097(7)$ & $4584(4)$ & $54(1)$ \\
C(13) & $7988(4)$ & $3758(7)$ & $4157(5)$ & $64(2)$ \\
C(14) & $8021(4)$ & $3655(7)$ & $3133(4)$ & $60(1)$ \\
C(15) & $8096(4)$ & $5167(8)$ & $2667(4)$ & $59(1)$ \\
C(16) & $8018(4)$ & $6486(7)$ & $3086(4)$ & $54(1)$ \\
C(17) & $8724(4)$ & $1190(8)$ & $2908(4)$ & $60(1)$ \\
C(18) & $9590(5)$ & $459(9)$ & $2771(6)$ & $80(2)$ \\
& & & & \\
\hline & & & & \\
\hline
\end{tabular}


Table 3. Bond lengths [A] and angles [deg] for JH.

\begin{tabular}{|c|c|}
\hline I(1)-C(8) & $2.062(5)$ \\
\hline Cl(1)-C(12) & $1.713(6)$ \\
\hline $\mathbf{O}(1)-C(9)$ & $1.217(7)$ \\
\hline $\mathrm{O}(2)-\mathrm{C}(17)$ & $1.358(8)$ \\
\hline$O(2)-C(14)$ & $1.469(7)$ \\
\hline$O(3)-C(17)$ & $1.204(8)$ \\
\hline $\mathrm{N}(1)-\mathrm{C}(9)$ & $1.360(7)$ \\
\hline$N(1)-C(10)$ & $1.447(6)$ \\
\hline$N(1)-C(11)$ & $1.457(6)$ \\
\hline C(1)-C(6) & $1.325(14)$ \\
\hline $\mathrm{C}(1)-\mathrm{C}(2)$ & $1.409(16)$ \\
\hline $\mathbf{C}(\mathbf{1})-\mathbf{H}(\mathbf{1})$ & 0.9300 \\
\hline$C(2)-C(3)$ & $1.26(2)$ \\
\hline $\mathbf{C}(2)-\mathbf{H}(2)$ & 0.9300 \\
\hline C(3)-C(4) & $1.346(18)$ \\
\hline $\mathrm{C}(3)-\mathrm{H}(3)$ & 0.9300 \\
\hline C(4)-C(5) & $1.413(14)$ \\
\hline $\mathrm{C}(4)-\mathrm{H}(4)$ & 0.9300 \\
\hline C(5)-C(6) & $1.309(11)$ \\
\hline C(5)-H(5) & 0.9300 \\
\hline$C(6)-C(7)$ & $1.482(7)$ \\
\hline$C(7)-C(8)$ & $1.325(8)$ \\
\hline$C(7)-C(11)$ & 1.537(7) \\
\hline $\mathrm{C}(8)-\mathrm{C}(9)$ & $1.476(7)$ \\
\hline C(11)-C(12) & 1.511(8) \\
\hline C(11)-C(16) & $1.514(7)$ \\
\hline $\mathrm{C}(10)-\mathrm{H}(10 \mathrm{~A})$ & 0.9600 \\
\hline $\mathrm{C}(10)-\mathrm{H}(10 \mathrm{~B})$ & 0.9600 \\
\hline C(10)-H(10C) & 0.9600 \\
\hline C(12)-C(13) & $1.320(9)$ \\
\hline C(13)-C(14) & $1.489(9)$ \\
\hline C(13)-H(13) & 0.9300 \\
\hline C(14)-C(15) & $1.480(10)$ \\
\hline C(14)-H(14) & 0.9800 \\
\hline C(15)-C(16) & $1.302(9)$ \\
\hline C(15)-H(15) & 0.9300 \\
\hline C(16)-H(16) & 0.9300 \\
\hline C(17)-C(18) & $1.475(9)$ \\
\hline C(18)-H(18A) & 0.9600 \\
\hline C(18)-H(18B) & 0.9600 \\
\hline C(18)-H(18C) & 0.9600 \\
\hline $\mathrm{C}(17)-\mathrm{O}(2)-\mathrm{C}(14)$ & 117.2(5) \\
\hline C(9)-N(1)-C(10) & $123.8(4)$ \\
\hline C(9)-N(1)-C(11) & $112.8(4)$ \\
\hline $\mathrm{C}(10)-\mathrm{N}(1)-\mathrm{C}(11)$ & 123.2(4) \\
\hline $\mathrm{C}(6)-\mathrm{C}(1)-\mathrm{C}(2)$ & 118.9(14) \\
\hline $\mathrm{C}(6)-\mathrm{C}(1)-\mathrm{H}(\mathbf{1})$ & 120.6 \\
\hline
\end{tabular}




\begin{tabular}{|c|c|}
\hline$C(2)-C(1)-H(1)$ & 120.6 \\
\hline C(3)-C(2)-C(1) & $125.3(15)$ \\
\hline $\mathrm{C}(3)-\mathrm{C}(2)-\mathrm{H}(2)$ & 117.3 \\
\hline $\mathrm{C}(1)-\mathrm{C}(2)-\mathrm{H}(2)$ & 117.3 \\
\hline$C(2)-C(3)-C(4)$ & 114.2(11) \\
\hline $\mathrm{C}(2)-\mathrm{C}(3)-\mathrm{H}(3)$ & 122.9 \\
\hline $\mathrm{C}(4)-\mathrm{C}(3)-\mathrm{H}(3)$ & 122.9 \\
\hline$C(3)-C(4)-C(5)$ & $120.8(11)$ \\
\hline $\mathrm{C}(3)-\mathrm{C}(4)-\mathrm{H}(4)$ & 119.6 \\
\hline $\mathrm{C}(5)-\mathrm{C}(4)-\mathrm{H}(4)$ & 119.6 \\
\hline$C(6)-C(5)-C(4)$ & 121.2(11) \\
\hline $\mathrm{C}(6)-\mathrm{C}(5)-\mathrm{H}(5)$ & 119.4 \\
\hline $\mathrm{C}(4)-\mathrm{C}(5)-\mathrm{H}(5)$ & 119.4 \\
\hline $\mathrm{C}(5)-\mathrm{C}(6)-\mathrm{C}(1)$ & $116.3(8)$ \\
\hline$C(5)-C(6)-C(7)$ & $121.0(7)$ \\
\hline$C(1)-C(6)-C(7)$ & $120.6(7)$ \\
\hline$C(8)-C(7)-C(6)$ & 129.2(5) \\
\hline$C(8)-C(7)-C(11)$ & $108.6(4)$ \\
\hline$C(6)-C(7)-C(11)$ & $122.2(5)$ \\
\hline$C(7)-C(8)-C(9)$ & 111.0(5) \\
\hline$C(7)-C(8)-I(1)$ & $129.5(4)$ \\
\hline$C(9)-C(8)-I(1)$ & 119.4(4) \\
\hline $\mathrm{O}(1)-\mathrm{C}(9)-\mathrm{N}(1)$ & $126.4(5)$ \\
\hline$O(1)-C(9)-C(8)$ & $127.8(5)$ \\
\hline N(1)-C(9)-C(8) & $105.8(4)$ \\
\hline$N(1)-C(11)-C(12)$ & $110.3(4)$ \\
\hline N(1)-C(11)-C(16) & $109.6(4)$ \\
\hline$C(12)-C(11)-C(16)$ & 111.2(5) \\
\hline $\mathrm{N}(1)-\mathrm{C}(11)-\mathrm{C}(7)$ & 101.7(4) \\
\hline$C(12)-C(11)-C(7)$ & $111.4(4)$ \\
\hline $\mathrm{C}(16)-\mathrm{C}(11)-\mathrm{C}(7)$ & $112.2(4)$ \\
\hline $\mathrm{N}(1)-\mathrm{C}(10)-\mathrm{H}(10 \mathrm{~A})$ & 109.5 \\
\hline N(1)-C(10)-H(10B) & 109.5 \\
\hline H(10A)-C(10)-H(10B) & 109.5 \\
\hline N(1)-C(10)-H(10C) & 109.5 \\
\hline $\mathrm{H}(10 \mathrm{~A})-\mathrm{C}(10)-\mathrm{H}(10 \mathrm{C})$ & 109.5 \\
\hline H(10B)-C(10)-H(10C) & 109.5 \\
\hline $\mathrm{C}(13)-\mathrm{C}(12)-\mathrm{C}(11)$ & $124.2(5)$ \\
\hline $\mathrm{C}(13)-\mathrm{C}(12)-\mathrm{Cl}(1)$ & $121.0(5)$ \\
\hline $\mathrm{C}(11)-\mathrm{C}(12)-\mathrm{Cl}(1)$ & $114.8(4)$ \\
\hline C(12)-C(13)-C(14) & 122.1(6) \\
\hline $\mathrm{C}(12)-\mathrm{C}(13)-\mathrm{H}(13)$ & 118.9 \\
\hline $\mathrm{C}(14)-\mathrm{C}(13)-\mathrm{H}(13)$ & 118.9 \\
\hline $\mathrm{O}(2)-\mathrm{C}(14)-\mathrm{C}(15)$ & 106.7(5) \\
\hline $\mathrm{O}(2)-\mathrm{C}(14)-\mathrm{C}(13)$ & 108.7(5) \\
\hline$C(15)-C(14)-C(13)$ & 114.6(5) \\
\hline O(2)-C(14)-H(14) & 108.9 \\
\hline$C(15)-C(14)-H(14)$ & 108.9 \\
\hline $\mathrm{C}(13)-\mathrm{C}(14)-\mathrm{H}(14)$ & 108.9 \\
\hline$C(16)-C(15)-C(14)$ & $122.7(5)$ \\
\hline $\mathrm{C}(16)-\mathrm{C}(15)-\mathrm{H}(15)$ & 118.6 \\
\hline C(14)-C(15)-H(15) & 118.6 \\
\hline
\end{tabular}




$\begin{array}{cc}\text { C(15)-C(16)-C(11) } & 124.6(5) \\ \text { C(15)-C(16)-H(16) } & 117.7 \\ \text { C(11)-C(16)-H(16) } & 117.7 \\ \text { O(3)-C(17)-O(2) } & 122.7(6) \\ \text { O(3)-C(17)-C(18) } & 126.9(6) \\ \text { O(2)-C(17)-C(18) } & 110.4(6) \\ \text { C(17)-C(18)-H(18A) } & 109.5 \\ \text { C(17)-C(18)-H(18B) } & 109.5 \\ \text { H(18A)-C(18)-H(18B) } & 109.5 \\ \text { C(17)-C(18)-H(18C) } & 109.5 \\ \text { H(18A)-C(18)-H(18C) } & 109.5 \\ \text { H(18B)-C(18)-H(18C) } & 109.5\end{array}$

Symmetry transformations used to generate equivalent atoms: 
Table 4. Anisotropic displacement parameters $\left(A^{\wedge} \wedge 2 \times 10 \wedge 3\right)$ for $\mathrm{JH}$. The anisotropic displacement factor exponent takes the form:

$$
-2 \text { pi^2 [ h^2 a*^2 U11 + ... + } 2 \text { h k a* b* U12 ] }
$$

\begin{tabular}{|c|c|c|c|c|c|c|}
\hline & U11 & U22 & U33 & U23 & U13 & U12 \\
\hline I(1) & 49(1) & 77(1) & 85(1) & $-13(1)$ & $10(1)$ & $12(1)$ \\
\hline Cl(1) & $90(1)$ & 97(1) & $57(1)$ & $10(1)$ & $18(1)$ & $0(1)$ \\
\hline $\mathbf{O}(1)$ & $48(2)$ & $75(3)$ & 75(3) & $-21(2)$ & $5(2)$ & $-10(2)$ \\
\hline $\mathbf{O}(2)$ & $45(2)$ & $64(2)$ & 89(3) & $-16(2)$ & $9(2)$ & $-3(2)$ \\
\hline $\mathrm{O}(3)$ & 77(3) & 63(3) & 88(3) & $-3(2)$ & 14(3) & $-8(2)$ \\
\hline $\mathbf{N}(\mathbf{1})$ & 35(2) & $58(3)$ & 54(2) & $-6(2)$ & $3(2)$ & $-1(2)$ \\
\hline $\mathrm{C}(1)$ & $99(8)$ & $340(20)$ & $150(11)$ & 73(13) & $-72(8)$ & $-93(11)$ \\
\hline $\mathrm{C}(2) \quad 110$ & 10(10) & $420(30)$ & $172(14)$ & 21(17) & $-79(11)$ & $-94(16)$ \\
\hline C(3) & $42(4)$ & 177(11) & 159(10) & $-56(9)$ & $-17(5)$ & $-17(5)$ \\
\hline$C(4)$ & 64(5) & $108(8)$ & 286(19) & $-22(10)$ & $53(8)$ & $-18(5)$ \\
\hline C(5) & 74(5) & 106(6) & 137(8) & $-15(6)$ & 11(5) & $-16(5)$ \\
\hline $\mathbf{C}(\mathbf{6})$ & 35(3) & $78(4)$ & 69(4) & $-7(3)$ & $-1(2)$ & $-3(2)$ \\
\hline C(7) & $33(2)$ & 68(3) & $52(3)$ & $0(3)$ & $-1(2)$ & $2(2)$ \\
\hline $\mathrm{C}(\mathbf{8})$ & $35(2)$ & $60(3)$ & $52(3)$ & $-2(2)$ & $6(2)$ & $6(2)$ \\
\hline $\mathrm{C}(9)$ & 41(3) & $60(3)$ & $46(3)$ & $-2(2)$ & $3(2)$ & $-4(2)$ \\
\hline $\mathrm{C}(\mathbf{1 1})$ & $36(2)$ & $54(3)$ & $52(3)$ & $-4(2)$ & $2(2)$ & $0(2)$ \\
\hline$C(10)$ & $38(3)$ & $74(4)$ & $72(4)$ & $-10(3)$ & $2(2)$ & $0(3)$ \\
\hline$C(12)$ & 46(3) & 62(3) & $56(3)$ & $3(3)$ & 11(2) & $-6(2)$ \\
\hline C(13) & $55(3)$ & $56(3)$ & $84(4)$ & $5(3)$ & 17(3) & $-8(3)$ \\
\hline$C(14)$ & $40(3)$ & $68(4)$ & 71(4) & $-20(3)$ & $6(2)$ & $-6(2)$ \\
\hline $\mathrm{C}(15)$ & $50(3)$ & $76(4)$ & 48(3) & $-3(3)$ & $3(2)$ & $0(3)$ \\
\hline$C(16)$ & $49(3)$ & 62(3) & $50(3)$ & $2(3)$ & $2(2)$ & $3(2)$ \\
\hline C(17) & 55(3) & $70(4)$ & $53(3)$ & $-6(3)$ & $3(2)$ & 1(3) \\
\hline$C(18)$ & $71(4)$ & 83(5) & $86(5)$ & $-11(4)$ & $6(4)$ & $20(3)$ \\
\hline
\end{tabular}


Table 5. Hydrogen coordinates $(x 10 \wedge 4)$ and isotropic displacement parameters $\left(A^{\wedge} 2 \times 10 \wedge 3\right)$ for $J H$.

\begin{tabular}{ccccc}
\hline & & & \\
& & & \\
& & & \\
& & & & \\
H(1) & 6206 & 7343 & 2483 & 247 \\
H(2) & 4736 & 6580 & 1898 & 294 \\
H(3) & 3840 & 5461 & 2777 & 155 \\
H(4) & 4156 & 6129 & 4350 & 180 \\
H(5) & 5606 & 7008 & 4976 & 127 \\
H(10A) & 9910 & 8205 & 4909 & 93 \\
H(10B) & 9717 & 6889 & 4154 & 93 \\
H(10C) & 9684 & 6522 & 5212 & 93 \\
H(13) & $\mathbf{8 0 1 5}$ & 2849 & 4508 & 77 \\
H(14) & 7474 & 3120 & 2824 & 72 \\
H(15) & 8203 & 5176 & 2049 & 70 \\
H(16) & $\mathbf{8 0 4 1}$ & 7388 & 2737 & 65 \\
H(18A) & 9983 & 375 & 3364 & 121 \\
H(18B) & 9880 & 1081 & 2352 & 121 \\
H(18C) & 9471 & -556 & 2507 & 121 \\
\hline
\end{tabular}


Table 6. Torsion angles [deg] for JH.

\begin{tabular}{|c|c|}
\hline$C(6)-C(1)-C(2)-C(3)$ & $-2(4)$ \\
\hline$C(1)-C(2)-C(3)-C(4)$ & 15(4) \\
\hline$C(2)-C(3)-C(4)-C(5)$ & $-13(2)$ \\
\hline C(3)-C(4)-C(5)-C(6) & $-1.7(18)$ \\
\hline$C(4)-C(5)-C(6)-C(1)$ & 15.1(16) \\
\hline$C(4)-C(5)-C(6)-C(7)$ & $178.6(8)$ \\
\hline$C(2)-C(1)-C(6)-C(5)$ & $-14(3)$ \\
\hline$C(2)-C(1)-C(6)-C(7)$ & $-177.1(17)$ \\
\hline$C(5)-C(6)-C(7)-C(8)$ & $-53.8(11)$ \\
\hline C(1)-C(6)-C(7)-C(8) & 109.0(13) \\
\hline$C(5)-C(6)-C(7)-C(11)$ & 124.9(8) \\
\hline$C(1)-C(6)-C(7)-C(11)$ & $-72.3(13)$ \\
\hline $\mathrm{C}(6)-\mathrm{C}(7)-\mathrm{C}(8)-\mathrm{C}(9)$ & $-179.3(6)$ \\
\hline$C(11)-C(7)-C(8)-C(9)$ & 1.8(6) \\
\hline $\mathrm{C}(6)-\mathrm{C}(7)-\mathrm{C}(8)-\mathrm{I}(1)$ & $2.4(9)$ \\
\hline C(11)-C(7)-C(8)-I(1) & $-176.5(4)$ \\
\hline $\mathrm{C}(10)-\mathrm{N}(1)-\mathrm{C}(9)-\mathrm{O}(1)$ & 4.6(9) \\
\hline $\mathrm{C}(11)-\mathrm{N}(1)-\mathrm{C}(9)-\mathrm{O}(1)$ & $-179.7(6)$ \\
\hline$C(10)-N(1)-C(9)-C(8)$ & $-175.6(5)$ \\
\hline $\mathrm{C}(11)-\mathrm{N}(1)-\mathrm{C}(9)-\mathrm{C}(8)$ & $0.1(6)$ \\
\hline$C(7)-C(8)-C(9)-O(1)$ & $178.6(6)$ \\
\hline $\mathrm{I}(1)-\mathrm{C}(8)-\mathrm{C}(9)-\mathrm{O}(1)$ & $-3.0(8)$ \\
\hline$C(7)-C(8)-C(9)-N(1)$ & $-1.2(6)$ \\
\hline $\mathrm{I}(\mathbf{1})-\mathrm{C}(\mathbf{8})-\mathrm{C}(\mathbf{9})-\mathrm{N}(\mathbf{1})$ & 177.2(3) \\
\hline C(9)-N(1)-C(11)-C(12) & $-117.4(5)$ \\
\hline $\mathrm{C}(10)-\mathrm{N}(1)-\mathrm{C}(11)-\mathrm{C}(12)$ & 58.3(7) \\
\hline C(9)-N(1)-C(11)-C(16) & $119.8(5)$ \\
\hline$C(10)-N(1)-C(11)-C(16)$ & $-64.5(6)$ \\
\hline $\mathrm{C}(9)-\mathrm{N}(1)-\mathrm{C}(11)-\mathrm{C}(7)$ & $0.9(6)$ \\
\hline $\mathrm{C}(10)-\mathrm{N}(1)-\mathrm{C}(11)-\mathrm{C}(\mathrm{7})$ & 176.6(5) \\
\hline $\mathrm{C}(8)-\mathrm{C}(7)-\mathrm{C}(11)-\mathrm{N}(1)$ & $-1.6(6)$ \\
\hline $\mathrm{C}(6)-\mathrm{C}(7)-\mathrm{C}(11)-\mathrm{N}(1)$ & 179.4(5) \\
\hline$C(8)-C(7)-C(11)-C(12)$ & 115.9(5) \\
\hline$C(6)-C(7)-C(11)-C(12)$ & $-63.1(7)$ \\
\hline$C(8)-C(7)-C(11)-C(16)$ & $-118.7(5)$ \\
\hline$C(6)-C(7)-C(11)-C(16)$ & 62.4(7) \\
\hline N(1)-C(11)-C(12)-C(13) & $-125.9(6)$ \\
\hline$C(16)-C(11)-C(12)-C(13)$ & $-4.0(8)$ \\
\hline $\mathrm{C}(7)-\mathrm{C}(11)-\mathrm{C}(12)-\mathrm{C}(13)$ & 121.9(6) \\
\hline $\mathrm{N}(1)-\mathrm{C}(11)-\mathrm{C}(12)-\mathrm{Cl}(1)$ & $54.9(5)$ \\
\hline$C(16)-C(11)-C(12)-C l(1)$ & $176.8(4)$ \\
\hline $\mathrm{C}(7)-\mathrm{C}(11)-\mathrm{C}(12)-\mathrm{Cl}(1)$ & $-57.3(5)$ \\
\hline$C(11)-C(12)-C(13)-C(14)$ & $-1.4(9)$ \\
\hline $\mathrm{Cl}(1)-\mathrm{C}(12)-\mathrm{C}(13)-\mathrm{C}(14)$ & $177.8(4)$ \\
\hline $\mathrm{C}(17)-\mathrm{O}(2)-\mathrm{C}(14)-\mathrm{C}(15)$ & $-147.1(5)$ \\
\hline $\mathrm{C}(17)-\mathrm{O}(2)-\mathrm{C}(14)-\mathrm{C}(13)$ & 88.9(6) \\
\hline $\mathrm{C}(12)-\mathrm{C}(13)-\mathrm{C}(14)-\mathrm{O}(2)$ & $126.8(6)$ \\
\hline$C(12)-C(13)-C(14)-C(15)$ & 7.6(8) \\
\hline
\end{tabular}




$$
\begin{array}{cc}
\text { O(2)-C(14)-C(15)-C(16) } & -128.9(6) \\
C(13)-C(14)-C(15)-C(16) & -8.6(8) \\
C(14)-C(15)-C(16)-C(11) & 3.3(9) \\
\text { N(1)-C(11)-C(16)-C(15) } & 125.4(6) \\
C(12)-C(11)-C(16)-C(15) & 3.1(7) \\
C(7)-C(11)-C(16)-C(15) & -122.4(6) \\
C(14)-O(2)-C(17)-O(3) & -0.1(9) \\
C(14)-O(2)-C(17)-C(18) & 179.7(5)
\end{array}
$$

Symmetry transformations used to generate equivalent atoms: 